\title{
GaN-based modulation doped FETs and UV detectors
}

\author{
Hadis Morkoç $^{\mathrm{a}, *}$, Aldo Di Carlo ${ }^{\mathrm{b}}$, Roberto Cingolani ${ }^{\mathrm{a}}$ \\ ${ }^{a}$ Department of Electrical Engineering and Physics, Virginia Commonwealth University, 601 W. Main Street, P.O. Box 843072, \\ Richmond, VA 23284-3072, USA \\ b INFM and Dept. Ingegneria Elettronica, Università di Roma "TorVergata", via di Tor Vergata n.110, 00133 Roma, Italy
}

Received 17 June 2001; accepted 18 June 2001

\begin{abstract}
GaN based modulation doped field effect transistors (MODFETs) and ultraviolet detectors are critically reviewed. $\mathrm{AlGaN} / \mathrm{GaN}$ MODFETs with $\mathrm{CW}$ power levels of about $6 \mathrm{~W}$ (in devices with $1 \mathrm{~mm}$ gate periphery) and a minimum noise figure of $0.85 \mathrm{~dB}$ with an associated gain of $11 \mathrm{~dB}$ have been obtained at $10 \mathrm{GHz}$. As a precursor to solar-blind detectors that will be operative around $280 \mathrm{~nm}$, where the solar radiation is absorbed by the ozone layer surrounding the earth, detector arrays with pixel sizes of $32 \times 32$ operative near the solar-blind region have been achieved. One does not have to rely on imagination to predict that devices with much improved performance will continue to be developed. (ㄷ 2002 Elsevier Science Ltd. All rights reserved.
\end{abstract}

Keywords: GaN; Nitride semiconductors; MODFETs; UV detectors; Solar blind detectors; High power FETs

\section{Contents}

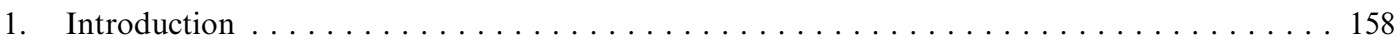

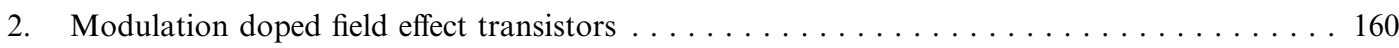

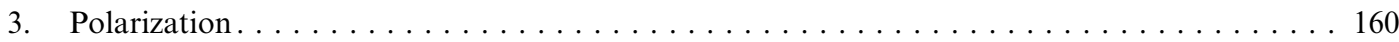

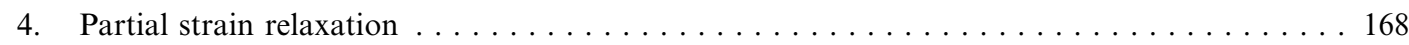

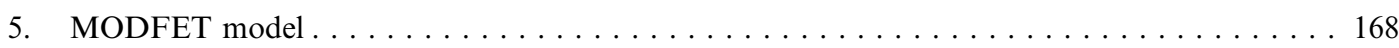

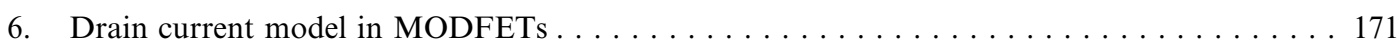

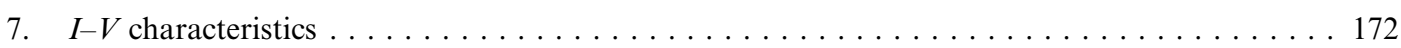

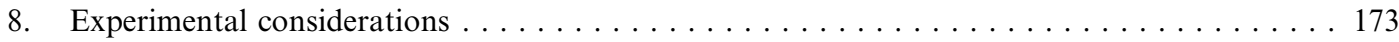

\footnotetext{
${ }^{*}$ Corresponding author. Permanent address: Department of Materials Science, University of Lecce, Lecce, Italy. Tel.: +1804-827-3765; fax: +1-804-828-4269.

E-mail address: hmorkoc@vcu.edu (H. Morkoç).

URL: http://www.vcu.edu/egrweb/vmc/research/index.html.
} 


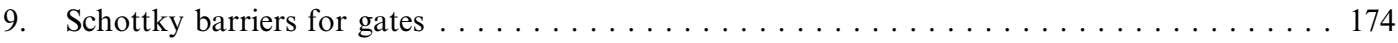

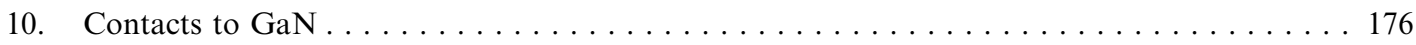

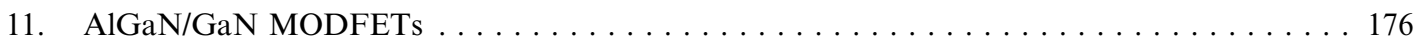

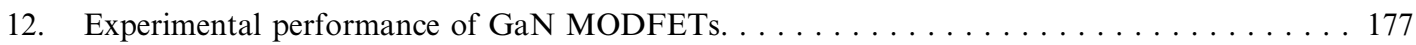

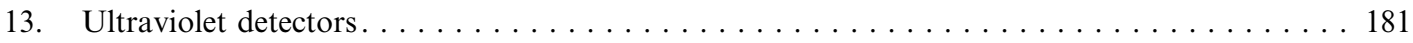

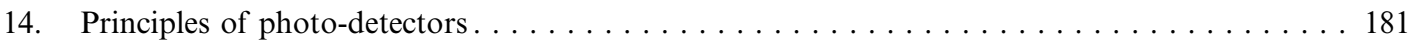

15. Current and voltage response to incident radiation $\ldots \ldots \ldots \ldots \ldots \ldots \ldots \ldots \ldots \ldots$

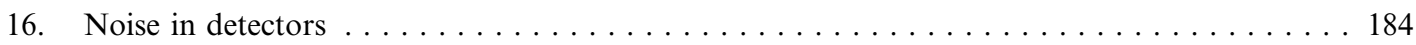

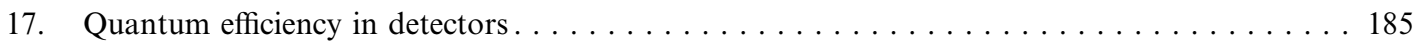

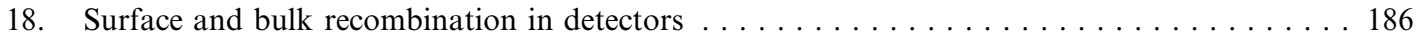

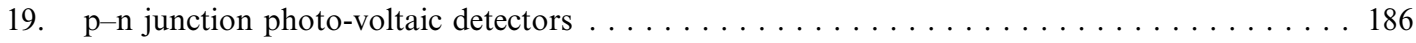

20. Diffusion current for a $\mathrm{p}-\mathrm{n}$ junction detector $\ldots \ldots \ldots \ldots \ldots \ldots \ldots \ldots \ldots \ldots \ldots \ldots \ldots$

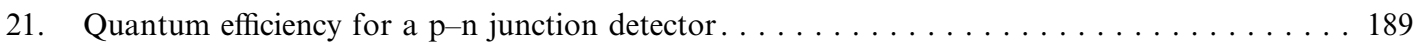

22. Noise in a $\mathrm{p}-\mathrm{n}$ junction detector . . . . . . . . . . . . . . . . . . . . 189

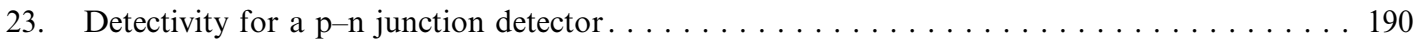

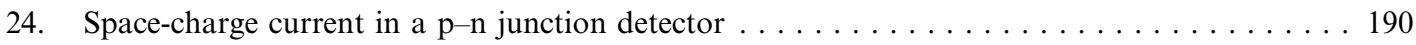

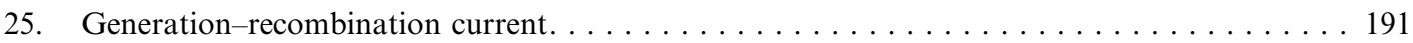

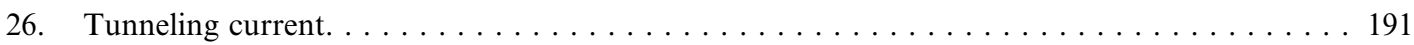

27. Surface leakage current. ................................ 191

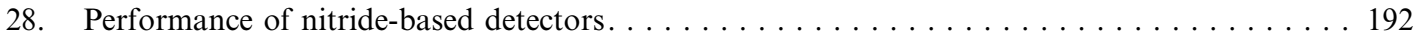

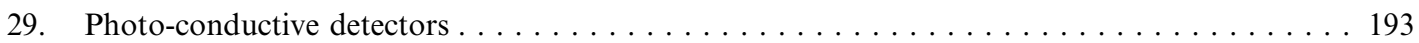

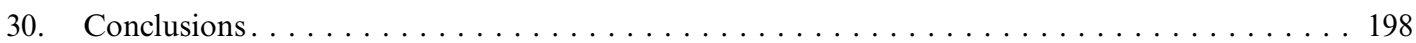

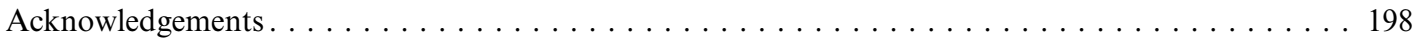

\section{Introduction}

Semiconductor nitrides such as aluminum nitride (AlN), gallium nitride $(\mathrm{GaN})$, and indium nitride $(\mathrm{InN})$ are very promising materials for their potential use in optoelectronic devices (both emitters and detectors), and high-power/temperature electronic devices as have been treated in length and reviewed recently [1-7]. These materials and their ternary and quaternary alloys cover an energy band gap range of $1.9-6.2 \mathrm{eV}$, suitable for band-to-band light generation with colors ranging from red (potentially) to ultraviolet (UV) wavelengths. Specifically, nitrides are suitable for such applications as surface acoustic wave devices [8], UV detectors $[9,10]$, 
Bragg reflectors [11], waveguides, UV and visible light emitting diodes (LEDs) [12-14], and laser diodes [15] for digital data read-write applications. During the last several decades, lasers and LEDs have expanded remarkably both in terms of the range of emission wavelengths available and brightness. The nitride semiconductor-based LEDs have proven to be reliable in such applications as displays, lighting, indicator lights, advertisement, and traffic signs/signals. Additional possible applications include use in agriculture as light sources for accelerated photosynthesis, and in health care for diagnosis and treatment. Lasers, as coherent sources, are crucial for high-density optical read and write technologies. Because the diffraction-limited optical storage density increases approximately quadratically (the best case scenario) as the probe laser wavelength is reduced, nitride-based coherent sources at wavelengths down to UV are attracting a good deal of attention. Optical storage would enable the storage and retrieval of inordinate number of images and vast quantities of text with untold efficiency. Other equally attractive applications envisioned include printing and surgery.

When used as UV sensors in jet engines, automobiles, and furnaces (boilers), the devices would allow optimal fuel efficiency and control of effluents for a cleaner environment. Moreover, UV sensors that operate in the solar blind region (260-290 $\mathrm{nm}$ ) would have high detectivity because the ozone layer absorbs solar radiation at those wavelengths, thus virtually eliminating the radiation noise. Consequently, these detectors are expected to play a pivotal role in threat recognition aimed against aircraft and other vehicles $[9,10,16]$. GaN photodiodes [17] exhibited zero-bias responsivities of about $0.21 \mathrm{~A} / \mathrm{W}$ at $356 \mathrm{~nm}$ that decreased by more than three orders of magnitude for wavelengths longer than 390 $\mathrm{nm}$. The noise equivalent power (NEP) at a reverse bias of $10 \mathrm{~V}$ is $(f>100 \mathrm{~Hz}) 6.6 \times 10^{-15} \mathrm{~W} / \mathrm{Hz}^{1 / 2}$ which is extremely small [18]. Detector speed affected in terms of uniformity by the sheet resistance of the p-layer, which suffers from the notoriously low doping levels, is in the picosecond range [19]. Finally, the GaN-based detectors with AlN mole fractions approaching the solar blind region of the spectrum have been fabricated into arrays for imaging. Detector arrays with pixel sizes of $32 \times 32$ have been fabricated and tested already $[18,20]$.

GaN's large band gap, large dielectric breakdown field, fortuitously good electron transport properties [21-23] (an electron mobility possibly in excess of 2000 $\mathrm{cm}^{2} \mathrm{~V}^{-1} \mathrm{~s}^{-1}$ and a peak velocity approaching $3 \times 10^{7} \mathrm{~cm} /$ $\mathrm{s}$ at room temperature), and good thermal conductivity are conducive for high power/temperature electronic devices [24]. Sheppard et al. [25] have reported that 0.45 $\mu \mathrm{m}$ gate, high-power modulation doped FETs (MODFETs) on SiC substrates exhibited a power density of 6.8 $\mathrm{W} / \mathrm{mm}$ in a $125 \mu \mathrm{m}$-wide device and a total power of $4 \mathrm{~W}$ (with a power density of $2 \mathrm{~W} / \mathrm{mm}$ ) at $10 \mathrm{GHz}$. Other groups have also reported on the superior performance of GaN-based MODFETs on SiC and sapphire substrates with respect to competing materials, particularly at $\mathrm{X}$ band and higher frequencies [26-29]. What is astounding is that researchers at HRL Laboratories have recently demonstrated GaN/AlGaN MODFETs prepared by molecular beam epitaxy (MBE) on SiC substrates, which exhibited a total power level of $6.3 \mathrm{~W}$ at $10 \mathrm{GHz}$ from a $1-\mathrm{mm}$ wide device. What is more astounding is that the power is not really thermally limited as the power density extrapolated from a $0.1-\mathrm{mm}$ device is $6.5 \mathrm{~W}$. Equally impressive is the noise figure of $0.85 \mathrm{~dB}$ at $10 \mathrm{GHz}$ with an associated gain of $11 \mathrm{~dB}$. The drain breakdown voltages in these quarter micron gate devices are about $60 \mathrm{~V}$, which are in part responsible for such a record performance [30].

Applications of high power GaN-based MODFETs include amplifiers operative at high power levels, high temperatures, and in unfriendly environments such as radar, missiles, satellites as well as in low-cost compact amplifiers for wireless base stations. Much of these applications are currently met by pseudomorphic MODFETs [31].

Nitride semiconductors have been deposited by vapor phase epitaxy (i.e., both hydride VPE [32] (HVPE) which has been developed for thick GaN layers and organometallic VPE [33] (OMVPE) which has been developed for heterostructures), and in vacuum by a host of variants of MBE [16]. All the high-performance light emitters, which require high-quality InGaN, have been produced by OMVPE. On the other hand, MBE has been very successful in producing structures, which do not require InGaN. Some examples are FETs and detectors. With its innate refined control of growth parameters, in situ monitoring capability, and uniformity, MBE is well suited for depositing heterostructures and gaining insight to deposition/incorporation mechanisms. MBE's control over growth parameters is such that any structure can be grown in any sequence. The structures based on conventional compound semiconductors such as IR lasers for CD players, surface emitting vertical cavity lasers, and high-performance pseudomorphic MODFETs have all been produced very successfully, most of them commercially, by MBE. Nitride growth, however, requires much higher temperatures than those used in producing conventional Group III-V semiconductors for which the MBE systems were designed. In addition, it has proved difficult to provide active $\mathrm{N}$ species at sufficiently high rates for nitride growth. Despite these mechanical/engineering limitations and its relatively late entry, with appropriate modifications, MBE has already played a key role on a number of fronts such as high-performance GaN-based MODFETs and fast solar blind detectors, which are the topics of discussion in this article. 


\section{Modulation doped field effect transistors}

With its reduced impurity scattering and unique gate capacitance-voltage characteristics, the MODFET has become the dominant high-frequency device. Among the MODFET's most attractive attributes are close proximity of the mobile charge to the gate electrode and high drain efficiency. As in the case of high emitters, the GaN-based MODFETs have quickly demonstrated record power levels at high frequencies with very respectable noise performance and large drain breakdown voltages.

In MODFETs, the carriers that form the channel in the smaller band gap material are donated by the larger band gap material and ohmic contacts or both. Since the mobile carriers and their parent donors are spatially separated, short-range ion scattering is nearly eliminated, which leads to mobilities that are characteristic of nearly pure semiconductors. A Schottky barrier is then used to modulate the mobile charge that in turn causes a change in the drain current. Because of this heterolayer construction, the gate can be placed very close to the conducting channel, resulting in large transconductances [34]. Fig. 1 presents a schematic representation of a GaN/AlGaN MODFET heterostructure in which the carriers are provided by the donors in the wider band gap AlGaN. In a MODFET device under bias, the carriers can also be provided by the source contact.

(a)

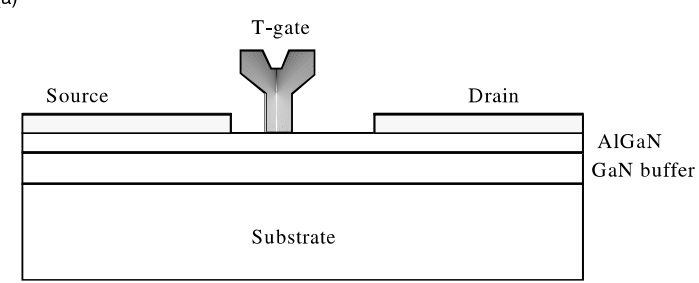

(b)

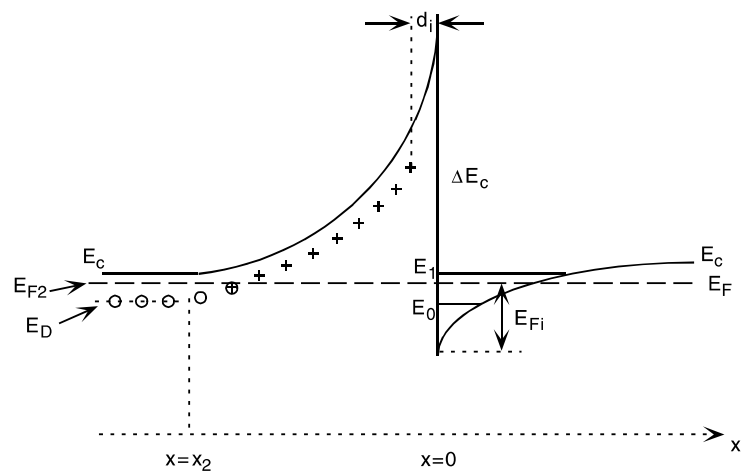

Fig. 1. (a) Schematic representation of an $\mathrm{AlGaN} / \mathrm{GaN}$ MODFET. (b) Schematic band structure of an AlGaN/GaN modulation doped heterostructure in which the free carriers are provided to the GaN layer by the dopant impurities placed in the larger band gap AlGaN barrier layer.

\section{Polarization}

The wurtzite Group III nitrides are tetrahedrally coordinated and lack the inversion symmetry along the $c$-direction. Because of this non-centrosymmetry and large ionicity associated with the covalent metal nitrogen bond, a large spontaneous polarization oriented along the hexagonal $c$-axis occurs. This spontaneous polarization is noteworthy particularly when heterointerfaces between two nitride semiconductors with varying ionicity are involved. Moreover, Group III nitride semiconductors have large piezoelectric (PE) coefficients. The strain induced PE and spontaneous polarization charges have profound effects on device structures. In heterojunction devices such as MODFETs where strain and heterointerfaces are present, the polarization charge is present and is inextricably connected to free carriers, which are indeed present. As such, polarization affects device operation in all nitride-based devices, particularly MODFETs, and thus must be taken into consideration in device design. As mentioned above, polarization charge arises from two sources: PE effects and the difference in spontaneous polarization between $\mathrm{AlGaN}$ and $\mathrm{GaN}$, even in the absence of strain. These charges exist in all compound semiconductors to varying degrees unless self-cancelled by the symmetry of the particular orientation under consideration. Spontaneous polarization has only recently been fully understood. As pointed out earlier [35], nitrides lack inversion symmetry and exhibit PE effects when strained along the [0001] direction. PE coefficients in nitrides are almost an order of magnitude larger than in many of the traditional Group III-V semiconductors [36]. In addition, wurtzite GaN has a unique axis, thus allowing spontaneous polarization $\left(P_{0}\right.$, whose values are given in Table 1$)$ to be present even in the absence of any strain. This can manifest itself as polarization charge at heterointerfaces. The magnitude of the polarization charge, converted to number of electrons can be in the mid- $10^{13} \mathrm{~cm}^{-2}$ level for AlN/GaN heterointerfaces, which is huge by any standard. For comparison, the interface charge in the GaAs/AlGaAs system used for MODFETs is less than $10 \%$ of this figure. An excellent review of the polarization effects can be found in Ref. [37].

Table 1

$\mathrm{PE}$ constants and spontaneous polarization charge in nitride semiconductors

\begin{tabular}{llll}
\hline & AlN & GaN & InN \\
\hline$e_{33}\left({\left.\mathrm{C} / \mathrm{m}^{2}\right)}^{2}\right.$ & 1.46 & 0.73 & 0.97 \\
$e_{31}\left(\mathrm{C} / \mathrm{m}^{2}\right)$ & -0.60 & -0.49 & -0.57 \\
$P_{0}\left(\mathrm{C} / \mathrm{m}^{2}\right)$ & -0.081 & -0.029 & -0.032 \\
{$\left[e_{31}-\left[C_{31} / C_{33}\right] e_{33}\right]$ units } & -0.86 & -0.68 & -0.90 \\
\hline
\end{tabular}


Let us compare the relative importance of spontaneous polarization to PE polarization. For a biaxially strained layer, the effective PE polarization is given by

$P_{z}^{\text {piezo }}=\left[e_{31}-\left(C_{31} / C_{33}\right) e_{33}\right] \varepsilon_{\perp}$

where $\varepsilon_{\perp}=\varepsilon_{x x}+\varepsilon_{y y}$ is the in-plane strain and $C_{31}$ and $C_{33}$ are elastic constants.

For coherently strained $\mathrm{Al}_{x} \mathrm{Ga}_{1-x} \mathrm{~N}$ on a relaxed $\mathrm{GaN}$ substrate, the strain $\varepsilon_{\perp}$ is expected to be proportional to $x$ and given by $\varepsilon=2\left(a_{\mathrm{GaN}}-a_{\mathrm{AlGaN}}\right) / a_{\mathrm{AlGaN}}$, which is $0.051 x$ and is tensile. The PE polarization is then $P^{\text {piezo }}=-0.044 x$, i.e., pointing in the direction [0001] . The corresponding difference in spontaneous polarization between $\mathrm{Al}_{x} \mathrm{Ga}_{1-x} \mathrm{~N}$ and $\mathrm{GaN}$ is also expected to be proportional to $x$, the AlN mole fraction, and is given by $\Delta P_{\text {spon }}=-0.052 x$. Consequently, the two are in the same direction for this particular orientation, and are comparable in magnitude. The total polarization for the AlN/GaN interface, which is defined in this case as the sum of the PE polarization and the differential polarization charge is $-0.096 x$. Note that these are all in $\mathrm{C} / \mathrm{m}^{2}$ and that $1 \mathrm{C} / \mathrm{m}^{2}=0.624 \times 10^{15}$ electrons $/ \mathrm{cm}^{2}$. Thus, for $x$ of the order of 0.1 , we are dealing with a total polarization charge of the order of mid- $10^{12} \mathrm{~cm}^{-2}$.

For a coherently strained $\operatorname{In}_{x} \mathrm{Ga}_{1-x} \mathrm{~N}$ layer on relaxed $\mathrm{GaN}$, the situation is different in that the difference in spontaneous polarization is much smaller, $\Delta P_{\text {spon }}=$ $-0.003 x$. Furthermore, the $\operatorname{In}_{x} \mathrm{Ga}_{1-x} \mathrm{~N}$ layer on $\mathrm{GaN}$ would be under compressive strain $\varepsilon_{\perp}=-0.203 x$, and $P^{\text {piezo }}=+0.183 x$. Here the PE polarization dominates and is opposite in direction to the spontaneous polarization charge, but even larger in absolute magnitude. In the case of a coherently strained $\mathrm{Al}_{x} \mathrm{In}_{1-x} \mathrm{~N}$ layer on a relaxed $\mathrm{GaN}$ layer, the situation is unique in that for $x=0$ we revert to the $\mathrm{InN}$ on $\mathrm{GaN}$ case and for $x=1$ we revert to the AlN on GaN case.

Numerical figures can be generated for the total polarization charge following the expressions outlined above by using a linear extrapolation of the strain and differential spontaneous polarization. The total polarization at the interface is the sum of the PE and differential spontaneous polarization, $P_{\text {total }}=\Delta P_{\mathrm{sp}}+P_{\mathrm{PE}}$. Taking the normal modulation doped structures where the GaN buffer layer is assumed completely relaxed and the $\mathrm{AlGaN}$ barrier layer is assumed coherently strained, one arrives at the plot shown in Fig. 2 for the total polarization charge at the interface. Additionally, data for InGaN and AlInN on GaN are shown. For tensilestrained $\mathrm{Al}_{x} \mathrm{Ga}_{1-x} \mathrm{~N}$ or $\mathrm{Al}_{x} \mathrm{In}_{1-x} \mathrm{~N}$ (for large $x$ values) on $\mathrm{GaN}$, the layer is under tensile strain and the PE and the spontaneous polarization are negative and point in the same direction, thus they add up. The spontaneous and PE polarizations oppose one another for compressively strained $\operatorname{In}_{x} \mathrm{Ga}_{1-x} \mathrm{~N}$ or $\mathrm{Al}_{x} \mathrm{In}_{1-x} \mathrm{~N}$ (for small $x$ values) layers. To calculate the differential spontaneous and PE
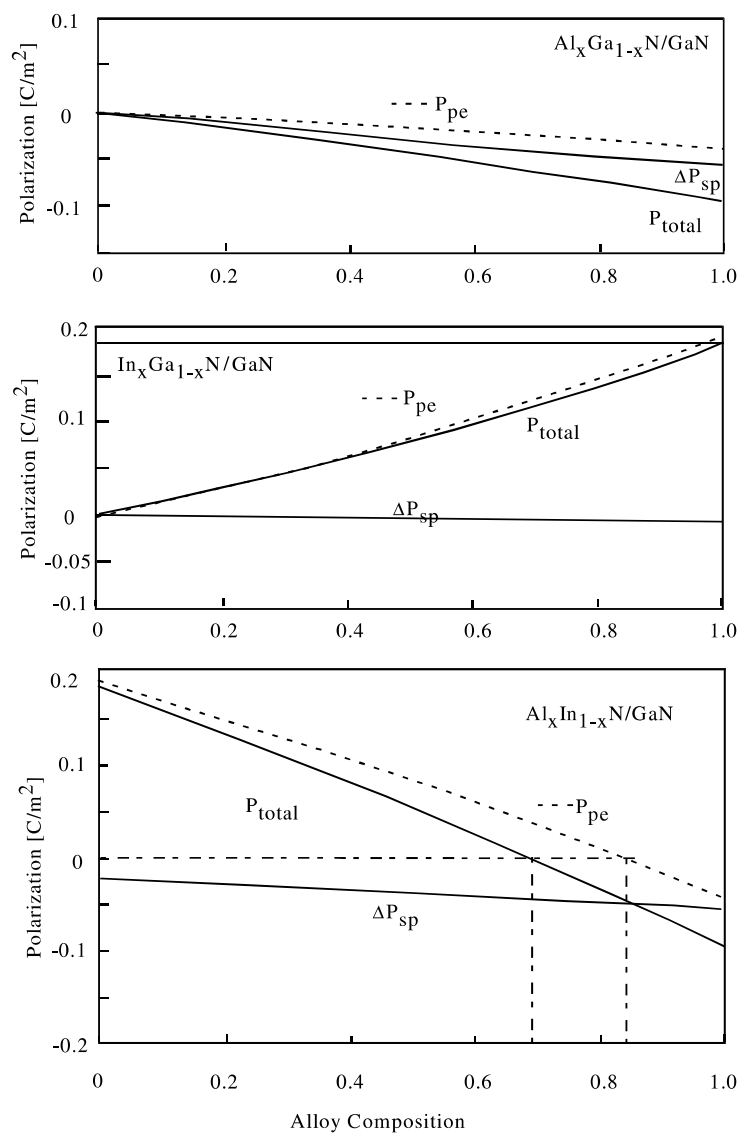

Fig. 2. PE, spontaneous, and total polarization of coherently strained AlGaN, InGaN and AlInN alloys grown on completely unstrained Ga-polarity GaN buffer layers vs. the alloy composition. The polarization values were determined by linear extrapolation of the physical properties from the binary compounds. The figure is similar to that reported by Ambacher et al. [38], but the parameters used are consistent with our previous publications.

polarization associated with alloys, one can employ a linear interpolation for the spontaneous polarization, $\mathrm{PE}$ and elastic constants from the binary compounds [38]. As discussed above, the PE polarization in coherently strained $\mathrm{Al}_{x} \mathrm{Ga}_{1-x} \mathrm{~N}$ layers grown on $\mathrm{GaN}$ increases to $-0.044 \mathrm{C} / \mathrm{m}^{2}$ for $x=1$. For InGaN layers, the PE polarization increases up to $+0.183 \mathrm{C} / \mathrm{m}^{2}$ for $x=1$. The ternary $\mathrm{Al}_{0.82} \mathrm{In}_{0.18} \mathrm{~N}$ can be grown lattice matched to $\mathrm{GaN}$ and the PE polarization vanishes. For lower $\mathrm{Al}$ concentrations, i.e., $x<0.82$, the PE polarization increases due to increasing biaxial compressive strain. For higher $\mathrm{Al}$ concentrations, i.e., $x>0.82$, the layer is under tensile strain and the PE polarization becomes negative (Fig. 2). Opposing strain and spontaneous polarization charge in $\mathrm{Al}_{x} \mathrm{In}_{1-x} \mathrm{~N}$ cancel one another for a mole fraction of about $x=0.7$ with the underlying assumption that the $\mathrm{Al}_{x} \mathrm{In}_{1-x} \mathrm{~N}$ is coherently strained 


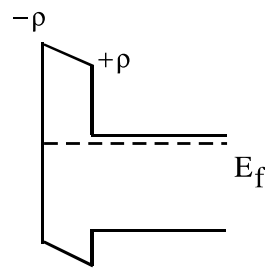

$\Delta \rho$

(a)

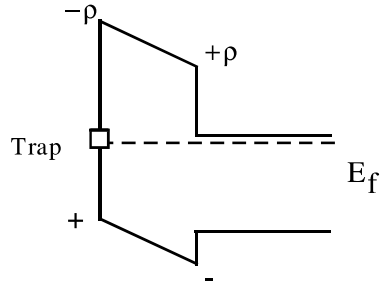

$\Delta \rho$

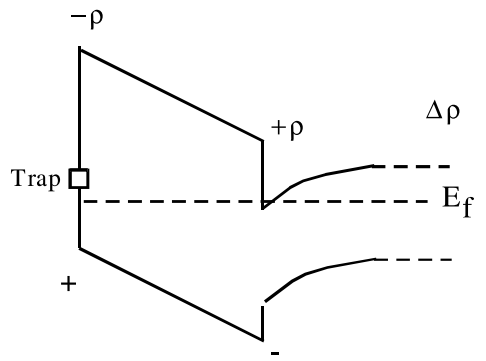

$\Delta \rho$

(b)

(c)

Fig. 3. Schematic representation of very simple-minded band structure of an AlGaN/GaN structure with varying AlGaN thickness, as one goes from (a)-(c), which demonstrates how the surface charge could participate in the screening of the polarization induced charge or the field.

and the GaN layer on which it is grown in completely relaxed [39]. The discussion of more accurate effective mass calculations can be found in the MODFET modeling section.

Some further words of caution about the above estimates are needed. If the AlGaN layers are not pseudomorphic but partially relaxed (by misfit dislocations for example), then the PE effect would be reduced but the spontaneous polarization would still be present. If the interfaces are not atomically sharp but exhibit a certain degree of inter-diffusion, the differences in spontaneous polarization would be reduced as well. Finally, if domains with inverted polarity exist, the overall polarization effects may be washed out. Also note that in an inverted structure with nitrogen $(\mathrm{N})$ polarity towards the surface, it may be possible to create a two-dimensional hole-gas at the AlGaN/substrate GaN interface, providing that free holes are available. However, if an n-type GaN layer is placed on top, a two-dimensional electron gas (2DEG) may form on top of the AlGaN layer.

Let us now consider a hypothetical case in which no free carriers exist. In this case, the polarization charge causes a linear band bending in $\mathrm{Al}_{x} \mathrm{Ga}_{1-x} \mathrm{~N}$ with no change in the band of the underlying $\mathrm{GaN}$ as shown in Fig. 3. Let us also assume that there are surface states below the conduction band within the band gap as shown. As the AlGaN thickness is increased, the band bending would be such that the surface states would become ionized. The released electrons in the process would wind up at the interface, which tend to screen the polarization charge. If the surface state concentration were not sufficiently high, the surface Fermi level comes very close to the valence band. This implies for holes to be present within the AlGaN layer near the surface. This positive charge needs to be balanced by a negative charge at the interface, the source of which could be surface states. In reality, the semiconductor system contains defects and free carriers that would make the picture somewhat more complicated. For one thing, the Fermi level may not be able to come as close to the valence band as that shown in the figure. The surface defect supposition has recently been forwarded with experimental backing [40].

With Ga polarity, the conduction band edge of the $\mathrm{Al}_{x} \mathrm{Ga}_{1-x} \mathrm{~N}$ will slope up toward the surface where the $\mathrm{Al}_{x} \mathrm{Ga}_{1-x} \mathrm{~N}$ layer is. The band diagram shown would hold in this hypothetical case only if the $\mathrm{Al}_{x} \mathrm{Ga}_{1-x} \mathrm{~N}$ layer thickness is such that the Fermi level at the surface is still a few $k T$ s away from the valence band edge. If the $\mathrm{Al}_{x} \mathrm{Ga}_{1-x} \mathrm{~N}$ thickness were made larger, the Fermi level near the surface would be very close to the valence band causing a positive hole charge at the surface as shown in Fig. 3. A negative image charge would then form at the heterointerface tending to screen the polarization charge. This charge is different from the polarization charge in that it is mobile and can partake in current flow in addition to altering the band diagram. In short, the polarization charge is a fixed charge. However, it would induce mobile charges to screen it if they are present. The recent device literature is confusing some authors implying as if the polarization charge alone is capable of doping a semiconductor. Misleading nomenclature with no physical basis such as "piezo doping" has already been coined. To make the point in another way, polarization charge causes band bending and any free carriers, if present in the system, would tend to screen the polarization charge. The screening charge represents the mobile carriers whose source could be native defects, impurity dopants (intentional or unintentional) and surface states.

Returning to modulation doped structures with $\mathrm{Al}_{x} \mathrm{Ga}_{1-x} \mathrm{~N}$ barriers, the sign of the polarization is as such to produce a potential energy for electrons sloping down from the Ga face toward the $\mathrm{N}$ face. Thus, for a structure in which the Ga face is turned toward the surface, the potential will slope down from the $\mathrm{Al}_{x} \mathrm{Ga}_{1-x} \mathrm{~N}$ surface toward the $\mathrm{AlGaN} / \mathrm{GaN}$ interface 
and help to drive carriers towards the 2DEG forming at this interface. For example, if there is an ohmic metal contact on the $\mathrm{Al}_{x} \mathrm{Ga}_{1-x} \mathrm{~N}$ surface, electrons will flow toward the 2DEG below that layer.

The most favorable situation for enhancing the sheet carrier concentration would occur for an InGaN quantum well on top of relaxed n-GaN and below an AlGaN barrier with the whole structure having cation polarity toward the surface. In that case, the field will slope down toward the InGaN/AlGaN interface in the quantum well and will help localize the carriers in the 2DEG. Note that the PE polarizations estimated here are based on the theoretical values for a perfectly insulating material. The free carriers that are present in each layer screen the field. For example, if free carriers are provided from metal contacts and they flow toward the 2DEG, this process sets up a screening field, which counters the polarization-induced field. Under equilibrium conditions, if they are reached, the net field is determined by the condition that the chemical potential for electrons (the Fermi level) must be constant throughout the structure. This depends on the doping and band bending in the substrate and, possibly, in each of the layers. At the very least, one may expect these fields to be reduced by a factor corresponding to the macroscopic dielectric constant, i.e., a factor of order 10 but possibly larger if the conductivity of the layers increases by the free carriers in the system. Consequently, a more realistic expectation for the effect on sheet carrier concentration is on the order of $10^{11}-10^{12}$ electrons $/ \mathrm{cm}^{2}$.

The difference between these and traditional device structures without polarization effects is that for uniform dopant concentrations, one obtains parabolically varying potentials with distance, whereas here the linear terms come from polarization on top of the parabolic terms. These linear terms lead to variations of the potential over a shorter distance scale determined by the thickness of the layers, whereas the parabolic terms correspond to the space-charge regions. Thus the linear terms may help to localize carriers if the polarity of the structure is chosen properly.

The immediate impact of this polarization is that the field generated by this process must be considered together with that induced by the applied voltage and charge redistribution. Moreover, as alluded to earlier, free carriers can also be drawn from any shallow and weakly bound impurities and metal in contact with the semiconductor. In any case, the free carriers would tend to screen the PE-induced polarization field. An additional complicating factor in nitrides in relation to polarization is that the semiconductor tends to twist and tilt in a columnar mode, in an effort to minimize strain as shown in Fig. 4. Multiple polarity has been confirmed in epitaxial GaN layers on sapphire substrates by convergent beam electron diffraction [41-43]. These columns do not have the same cation/anion ordering

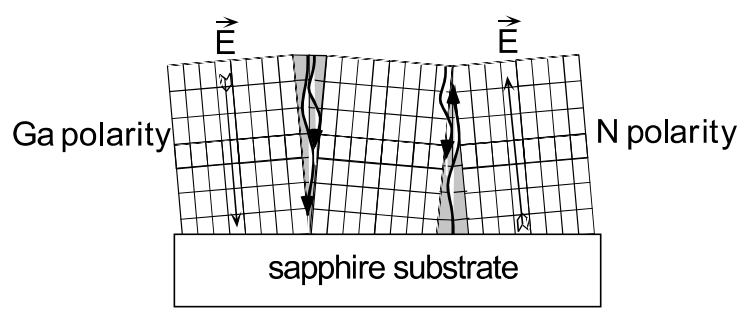

Fig. 4. Domains in $\mathrm{GaN}$, with $\mathrm{N}$ polarity (nitrogen surface layer) on the right and Ga polarity (with Ga on surface) on the left side under compressive residual strain. The arrows show the direction of the PE field in each of the domains.

polarity as shown in Fig. 4. In the presence of strain, Ga polarity domains and $\mathrm{N}$ polarity domains would have opposite polarization, causing increased scattering.

Fig. 5 is a schematic representation of an ideal inversion domain boundary formed in growth along the [0001] direction. On the left of the boundary, the growth initiates with $\mathrm{N}$, and on the right it begins with $\mathrm{Ga}$. On the left side, the bond along the [0001] direction is from Ga to N; this is called Ga polarity. On the right side, the [0001] bonds are from $\mathrm{N}$ to $\mathrm{Ga}$; this is called $\mathrm{N}$ polarity. In $\mathrm{N}$ polarity and under tensile strain, the $\mathrm{PE}$ field generated points toward the surface, whereas that for the Ga polarity region points in from the surface. When the strain is compressive, the direction of the field changes.

Yet an additional complicating factor is the asymmetry in the barrier discontinuities between $\mathrm{GaN}$ and its binary and ternaries caused by spontaneous polarization [44-46]. The spontaneous polarization arises simply because of the ionicity of the bonds and the low symmetry in wurtzite. In fact, Bernardini and Fiorentini [47] showed that the field that occurs in quantum wells is almost completely determined by the difference in spontaneous polarization between the two bulks, and their PE contribution. The field (i.e., the slope of the potential) is quite independent of the offset (i.e., the dipole discontinuity that occurs at the interface between the two materials). In other words, the dipole at the interface appears to be independent from the charge (monopole) accumulated at the interface. Recent experimental results obtained in the authors' laboratory with colleagues from the University of Lecce support this theory in that the spontaneous polarization component is dominant.

In considering a normal MODFET (N-MODFET) structure where the larger band gap $\mathrm{AlGaN}$ donor layer is deposited on top of a GaN channel layer (see Fig. 6) both the spontaneous polarization and PE polarization must be accounted for. For an N-MODFET structure with $\mathrm{Ga}$ polarity, the potential will slope down from the surface of the AlGaN layer toward the AlGaN/GaN 


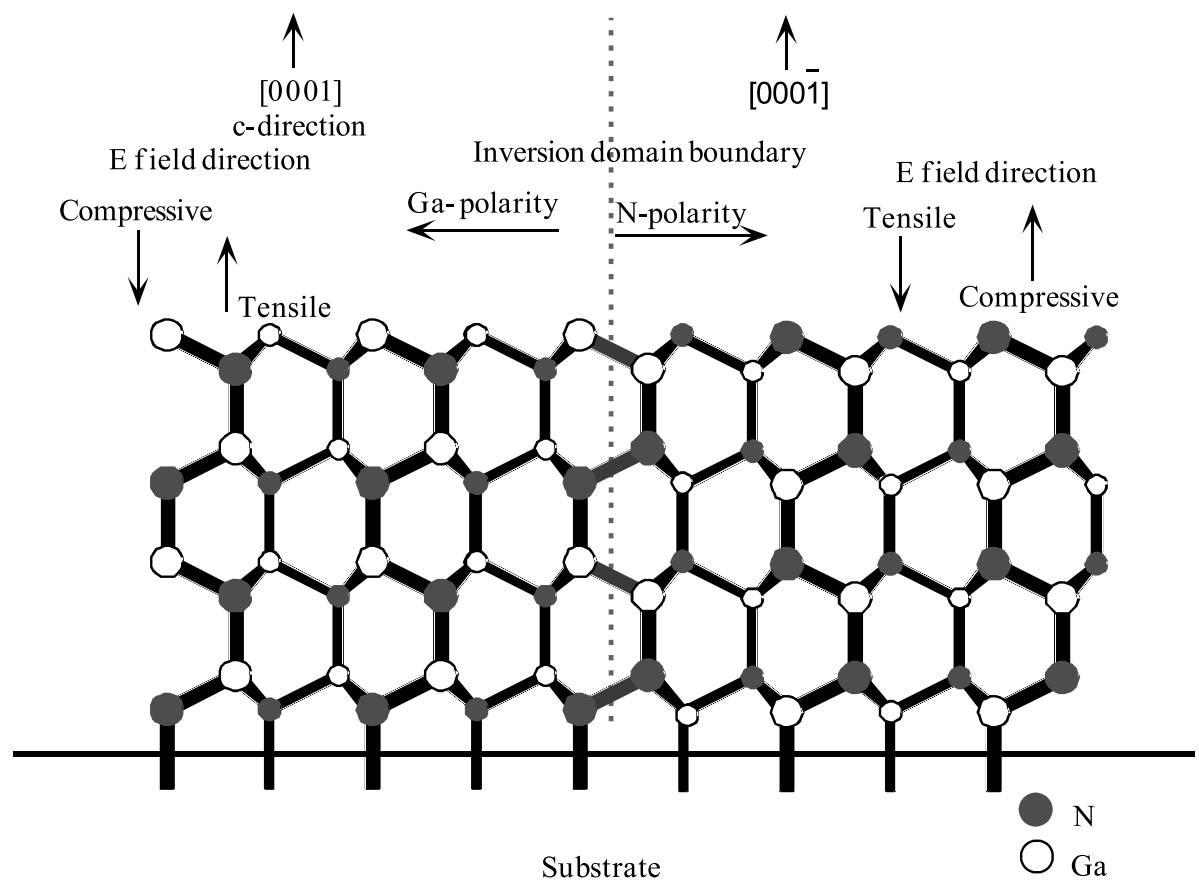

Fig. 5. Schematic representation of an ideal inversion domain boundary formed in along the $c$-axis.

$$
\stackrel{[0001]}{\text { Ga-Polarity }}
$$

$\mid$ sponAlGaNl>IPsponGaN|

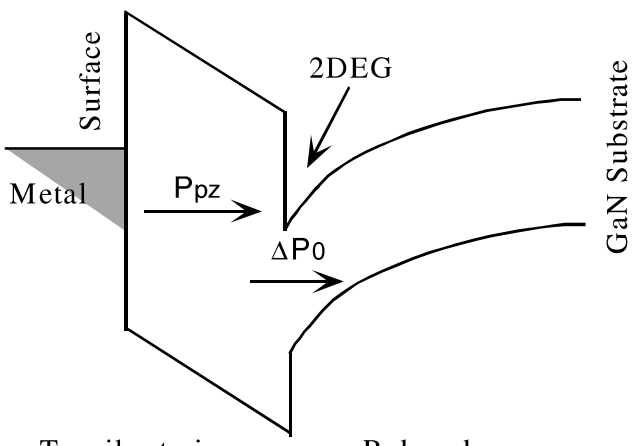

Tensile strain

Relaxed $\mid$ sponAIGaN $|>|$ sponlnGaNl

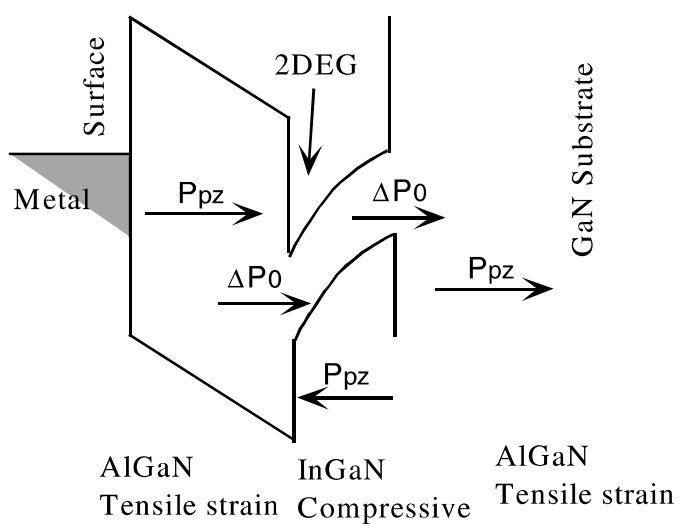

Fig. 6. GaN-based normal modulation doped structures with Ga polarity and GaN and InGaN active layers. If the signs of strain were to change, to compressive from tensile, then the direction of the PE polarization would change. In that case the spontaneous and PE polarization charges would oppose one another, and the larger one would determine whether hole or electron accumulation is favored at the interface.

interface, and will help to drive free electrons toward the interface forming a 2DEG as shown in Fig. 6. For example, if there is an ohmic metal contact on the AlGaN surface, electrons will flow toward the 2DEG below the
AlGaN layer from contacts. Since nitride semiconductors in question have large band gaps, thermal generation rates are minuscule and the role played by thermally generated carriers can be ignored. 


\section{$\leftarrow_{\text {N-Polarity }}^{[000 \overline{1}]}$}

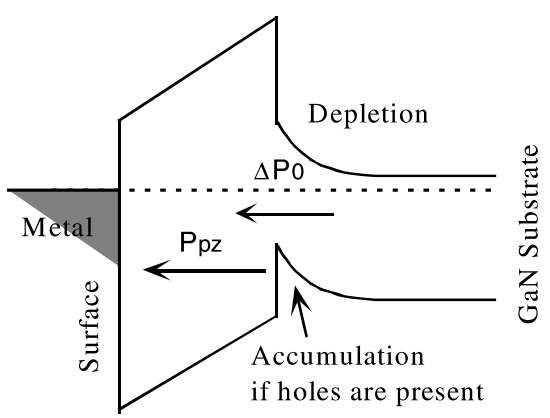

Tensile strain Relaxed

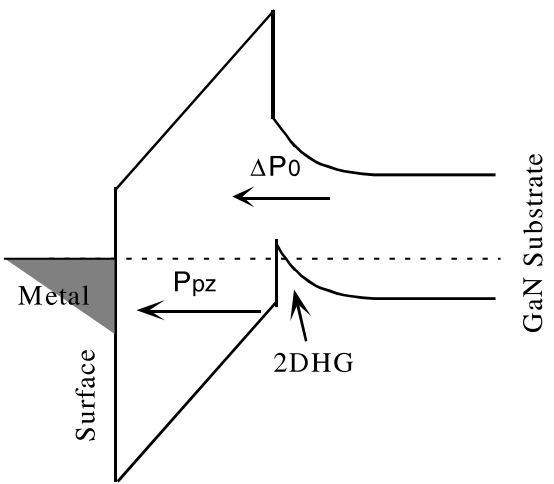

Tensile strain $\quad$ Relaxed

n-type GaN

-... Fermi level

Fig. 7. AlGaN/GaN-based normal modulation doped structures with $\mathrm{N}$ polarity with two cases, one for n-type and the other for p-type buffer layer. If the signs of strain were to change, to compressive from tensile, then the direction of the PE polarization would change. In that case the spontaneous and PE polarizations would oppose one another, and the larger one would determine whether hole or electron accumulation is favored at the interface.

It is therefore essential that the $\mathrm{N}$-face case also be considered. The case of an AlGaN (tensile-strained)/ $\mathrm{GaN}$ (relaxed) heterostructure with nitrogen polarity for an n-type $\mathrm{GaN}$ and for a p-type $\mathrm{GaN}$ buffer layers is shown in Fig. 7. In the cases depicted in Fig. 6, the PE polarization and spontaneous polarization charges support one another. Unlike the Ga polarity case, the polarization charge is such that screening charge will be made of holes if they are present in the film. If holes constitute the minority charge in the film, then the thermal process is the means by which they would be created. However, this process in a wide band gap semiconductor such as GaN is very slow and equilibrium condition may not be attained. If the strain in AlGaN were compressive, the direction of the PE polarization vector would change which would cause the PE polarization to counter the spontaneous polarization. This would actually represent the case when the epitaxial films are relaxed at the growth temperature, but upon cooling to room temperature, the film would be under compressive strain if on sapphire substrates. This is due to the expansion coefficient of sapphire being larger than that of GaN. In such a case, the larger of the two charges would dominate and determine whether hole or electron accumulation would be favored. If on the other hand the film is grown on a $\mathrm{SiC}$ substrate, the strain due to thermal expansion would be tensile.
This would lead to the case where the PE polarization and spontaneous polarization would support one another.

Inverted modulation doped structures can also be used to interrogate the picture in effect and perhaps take advantage of the unique features presented. In such a case, the $\mathrm{AlGaN}$ layer precedes the $\mathrm{GaN}$ top layer where the charge accumulation would occur. The interface between the AlGaN layer and the bottom GaN layer which is referred to as the buffer layer would be graded to avoid a normal interface.

Fig. 8 shows, schematically, AlGaN/GaN-based inverted modulation doped structures with $\mathrm{Ga}$, and $\mathrm{N}$ polarities. As can be seen, in the case of Ga polarity and tensile strain in $\mathrm{AlGaN}$, both the PE and spontaneous polarization vectors support each other, leading to a hole accumulation at the interface if holes are present in the system. Since the thermal generation rate is very small, the semiconductor structure cannot be expected to reach equilibrium by this means at room temperature in a reasonable period of time. On the other hand, with $\mathrm{N}$ polarity and tensile strain in $\mathrm{AlGaN}$, the structure favors electron accumulation at the interface. If the sign of strain were to change, to compressive from tensile, then the direction of the PE polarization would change. In that case the spontaneous and PE polarization charges would oppose one another, and the larger one 


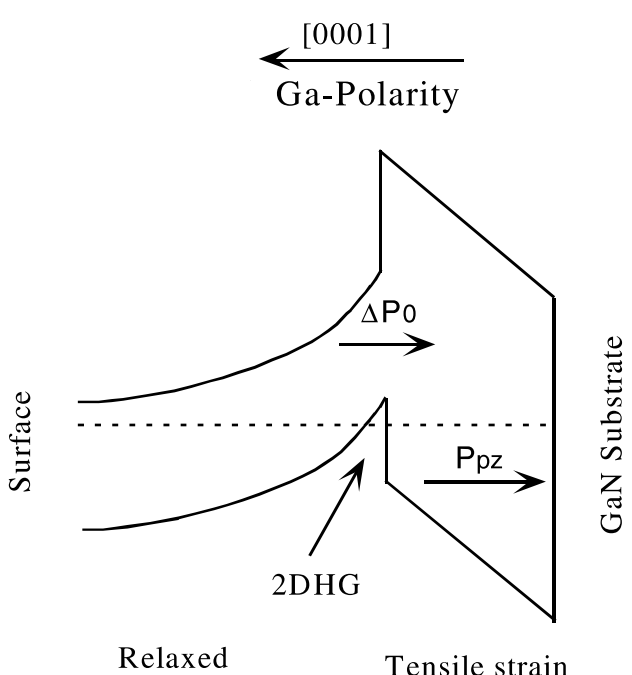

Hole accumulation if they are present

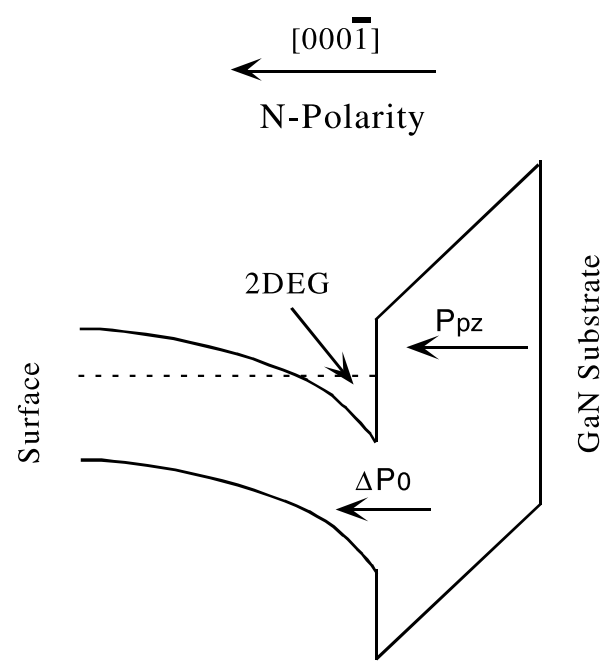

Relaxed Tensile strain

Electron accumulation

Fig. 8. AlGaN/GaN-based Inverted modulation doped structures with $\mathrm{Ga}$, and $\mathrm{N}$ polarity. As can be seen, in the case of Ga polarity and tensile strain in $\mathrm{AlGaN}$, both the $\mathrm{PE}$ and spontaneous polarization vectors support each other, leading to hole accumulation at the interface if holes are present in the system. The other source, thermal generation rate is very small and the semiconductor structure cannot be expected to reach equilibrium by this means at room temperature in a reasonable period of time. On the other hand, with $\mathrm{N}$ polarity and tensile strain in $\mathrm{AlGaN}$, the structure favors electron accumulation at the interface. If the signs of strain were to change, to compressive from tensile, then the direction of the PE polarization would change. In that case the spontaneous polarization and PE polarization would oppose one another, and the larger one would determine whether hole or electron accumulation is favored at the interface.

would determine whether hole or electron accumulation is favored at the interface.

Ambacher et al. [39] employing Hall effect and capacitance-voltage profiling measurements, measured the sheet carrier concentration and its profile in modulation doped structures at room temperature, the results of which are shown in Fig. 9. As seen in the figure, the sheet carrier concentrations are consistently larger than those expected from PE polarization alone. Since, the $\mathrm{GaN}$ buffer contribution was verified to be negligible, some additional sources, namely spontaneous polarization was invoked to explain the observations. The contribution from the unintentionally doped barrier layer also was found to be negligible as the measure of 2DEG concentration with increasing barrier thickness up to $650 \AA(x=0.25)$ did not appear to affect the results. The highest measured sheet carrier concentrations for $0.2<x<0.45$ are in good agreement with the calculated values of total polarization charge taking spontaneous polarization and the measured strain relaxation of $\mathrm{AlGaN}$ into account. The maximum sheet carrier concentration for undoped $\mathrm{AlGaN} / \mathrm{GaN}$ (and also for $\mathrm{InGaN} / \mathrm{GaN}$ ) heterostructures is limited to about $2 \times 10^{13} \mathrm{~cm}^{-2}$ due to strain relaxation of the top alloy layer. Not in good agreement are the calculated and measured sheet carrier concentrations of undoped MODFET structures with alloy compositions $x<0.2$. The measured sheet carrier concentrations are up to $5 \times 10^{12} \mathrm{~cm}^{-2}$ smaller than the predicted ones. In addition, the large scatter of the measured sheet carrier concentrations for heterostructures with similar barrier thicknesses and alloy compositions is unexpected and much larger than the error in experiments. For a more accurate calculation of the polarization charge in $\mathrm{Ga}$ polarity modulation doped structure, see the section on MODFET modeling.

Polarization effects, particularly spontaneous polarization, have immense impact on measured band discontinuities also for example, the dependence of measured band discontinuities on the order in which the larger and the smaller band gap semiconductors are grown, is one that can be attributed to polarization effects $[45,46]$. The complicating factor in nitrides is the universal lack of polarity control, (Ga or N polarity), and lack of uniform polarity, meaning presence of inversion domains. Though, layers grown by MBE with a RF activated nitrogen source turn out to be of $\mathrm{N}$ polarity and $\mathrm{Ga}$ polarity when $\mathrm{GaN}$ and $\mathrm{AlN}$ buffer layers are used, re- 


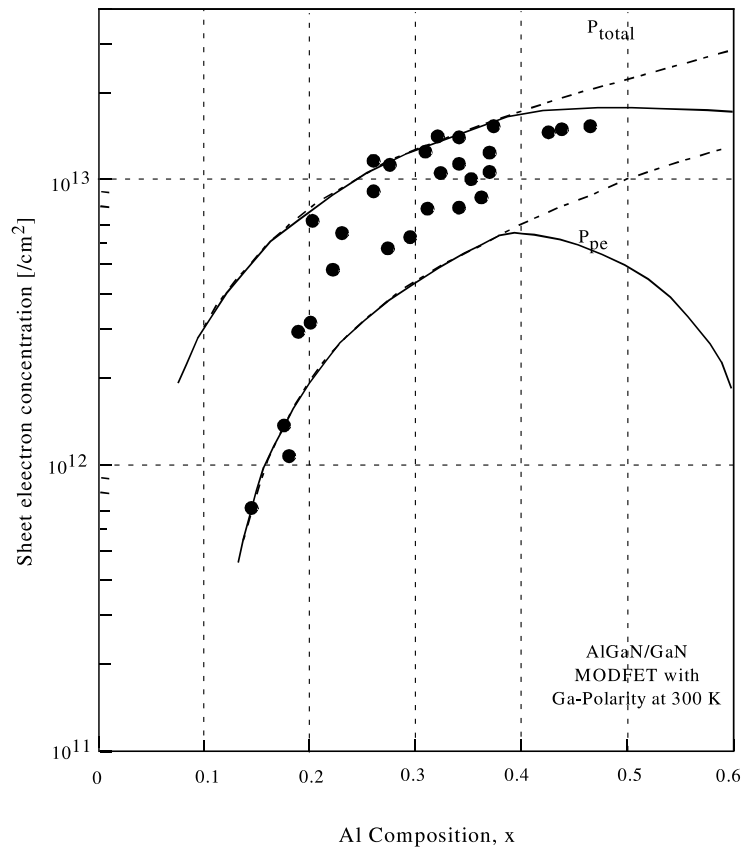

Fig. 9. Comparison of calculated and measured electron sheet carrier concentrations in Ga-face $\mathrm{AlGaN} / \mathrm{GaN}$ heterostructures. The dashed lines are calculated results for pseudomorphic structures. The solid lines are indicative of the calculated results, which take the measured partial strain relaxation into account (for more details, see Refs. [36,37]).

spectively, more investigations are needed to shed more light on the subject.

To reiterate, as a result of polarization, the static potential at the GaN/AlN interface is different from that at the $\mathrm{AlN} / \mathrm{GaN}$ interface that gives rise to interface charge larger than the charge densities used in devices. A substantial level of effort has been expended toward determining band discontinuities, but the field is in desperate need of more in-depth investigations in improved structures. The observed asymmetry in AlN/ $\mathrm{GaN}$ and GaN/AlN interfaces caused by spontaneous polarization is within the experimental errors of Martin et al. [44-46]. Inversion domains (see Fig. 10) combined with any strain in semiconductor nitrides lead to flipping PE fields (see Fig. 11) with adverse effects on our ability to characterize the films, let alone exploit this phenomenon for devices. Such flipping field would also cause increased scattering of carrier as they traverse in the $c$-plane. Simply put, identical device structures with different polarity layers would have widely-differing performance underscoring the importance that these issues must be investigated and reconciled. The polarity mixing causes the PE induced electric field to flip from one domain to the next, causing a variation in the sheet

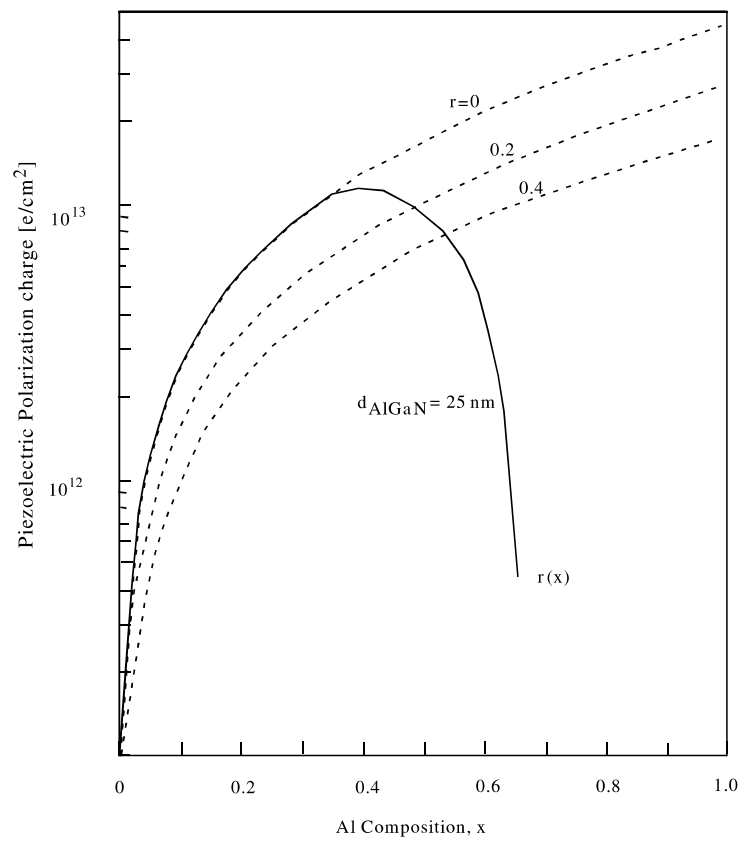

Fig. 10. PE polarization induced sheet charge at the interface of a Ga-face $\mathrm{AlGaN} / \mathrm{GaN}$ heterostructure for different degrees of relaxation of the barrier layer (dashed lines). The solid line was calculated taking into account the measured degree of relaxation, for $\mathrm{AlGaN}$ top layers with thicknesses of $25 \pm 5 \mathrm{~nm}$. The figures have been modified to be consistent with the PE and polarization charge figures used in our previous publications.

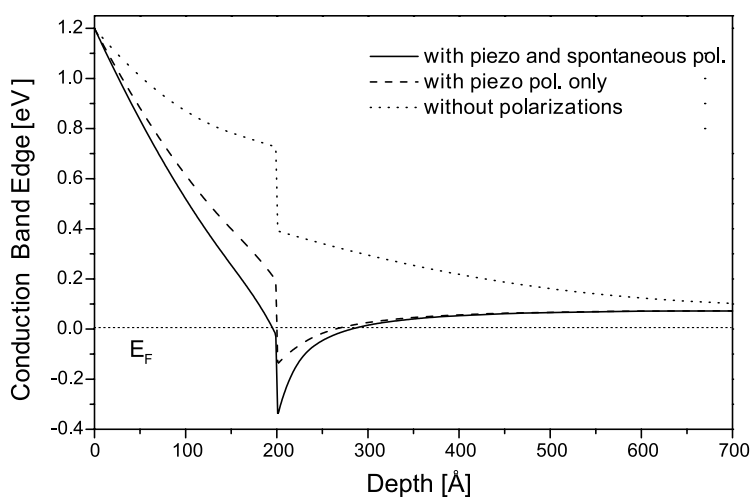

Fig. 11. Conduction band edge for the N-MODFET structure grown in the [0001] direction for $V_{\mathrm{G}}=0$ with and without polarization fields.

carrier concentration along the channel of an FET like device. The same polarity mixing would have deleterious effects in the base of an HBT as well, and depending on the polarity the induced field would either aid or impede minority carrier transit. 


\section{Partial strain relaxation}

The heretofore discussion dealt only with coherently strained AlGaN top layer and relaxed GaN buffer layer in the case of normal modulation doped structures and relaxed $\mathrm{AlGaN}$ and coherently-strained $\mathrm{GaN}$ top layer in the case of inverted modulation doped structures. This is of course an assumption whose validity has not been confirmed. In reality, a most likely scenario is that a partial relaxation would take place coupled with a residual thermal strain. Ambacher et al. [39] treated this issue of partial relaxation. As pointed out by Ambacher et al., calculations of the critical thickness of InGaN and AlGaN grown on GaN show that for a typical AlGaN barrier thickness of about $20 \mathrm{~nm}$, strain relaxation should occur for alloy compositions above $x=0.2$. At the outset, we should mention that the critical thickness, the thickness above which the film begins to relax, is difficult to determine because of the large density of dislocations in this material system. Nevertheless, proceeding as if it can be done, does indeed give an estimate, which is very useful. In order to determine the alloy composition and relaxation of the barrier, the lattice constants $a(x)$ and $c(x)$ were measured by high resolution X-ray diffraction [38]. For barrier thicknesses of about $25 \mathrm{~nm}$, macroscopic strain relaxation was observed for alloy compositions of $x_{\mathrm{c}}=0.20$ and 0.38 for InGaN and $\mathrm{AlGaN}$, respectively. In both cases the degree of relaxation increased nearly linearly with increasing alloy composition for $x>x_{\mathrm{c}}$. Naturally, for a barrier with a fixed alloy composition, the PE polarization and the sheet charge induced by that polarization decrease linearly with increasing level of relaxation $(r)$. The polarization induced charge at the interface for a $\mathrm{Ga}$ face $\mathrm{AlGaN} / \mathrm{GaN}$ heterostructure is shown vs. Al concentration for different degrees of strain relaxation in Fig. 10. The maximum sheet charge caused by PE polarization of a strained $30-\mathrm{nm}$ thick barrier was calculated using the measured degree of relaxation. As can be seen, for a barrier with $x=0.4$ the total sheet charge induced by spontaneous and PE polarization decreases if the AlGaN becomes partially or completely relaxed.

\section{MODFET model}

The Schrödinger equation and Poisson equation can be used self-consistently in order to study the channel formation and current flow mechanisms in GaN-based MODFET [34]. Several approaches have been used to define the system Hamiltonian used in the Schrödinger equation, namely effective masses [48], k.p expansion [49] and tight-binding expansion [50,51]. The use of sophisticated models such as k.p or tight binding is justified, perhaps even made necessary, by the complex wurtzite band structure, particularly for determining the valence band states. Thus, calculations of optical processes involving band-to-band transitions must consider the details of the band structure beyond the simple effective mass approximation (EMA) [52,53]. However, even when only the conduction band processes are of interest, EMA is still very accurate means of determining the properties of interest. In fact, nitride-based semiconductors in the wurtzite structure posses a conduction band with a $\Gamma$ minimum, which can be described reasonably well within such an approximation. Within the effective mass theory, Schrödinger equation takes the form $[34,48,54]$

$$
-\frac{h^{2}}{2} \frac{\mathrm{d}}{\mathrm{d} z}\left(\frac{1}{m(z)} \frac{\mathrm{d}}{\mathrm{d} z}\right) \varphi+e V(z) \varphi=E \varphi
$$

where $m(z)$ is the position dependent effective mass, $V(z)$ the electrostatic potential, $\varphi$ the electron wave function and $E$ the energy level. Non-parabolicity may induce deviations from the simple parabolic band model. However this will not substantially change the results that we will present.

In the nitride semiconductors with wurtzite phase, spontaneous and PE polarization effects are present [55], which necessitates that Poisson equation be solved for the displacement field, $D(z)$

$$
\begin{aligned}
\frac{\mathrm{d}}{\mathrm{d} z} D(z) & =\frac{\mathrm{d}}{\mathrm{d} z}\left(-\varepsilon(z) \frac{\mathrm{d}}{\mathrm{d} z} V(z)+P(z)\right) \\
& =e\left(p(z)-n(z)+N_{\mathrm{D}}^{+}-N_{\mathrm{A}}^{-}\right)
\end{aligned}
$$

where $\varepsilon(z)$ is the position-dependent dielectric constant, $P$ the total polarization, $n(p)$ the electron (holes) charge concentration and $N_{\mathrm{D}}^{+}\left(N_{\mathrm{A}}^{-}\right)$the ionized donor (acceptor) density.

In the self-consistent procedure, potential $V$ is obtained using Eq. (3) from an initial guess of the mobile charge concentration, and then inserted into the Schrödinger equation, Eq. (2), which is solved to get the energy levels and wave functions of the system. The new electron charge density is obtained by applying Fermi statistics as follows:

$n_{2 \mathrm{D}}(z)=\frac{m(z) k_{\mathrm{B}} T}{\pi \hbar^{2}} \sum_{i}\left|\varphi_{i}(z)\right|^{2} \ln \left[1+\mathrm{e}^{\left(E_{\mathrm{F}}-E_{i}\right) / k_{\mathrm{B}} T}\right]$

where $E_{\mathrm{F}}$ is the Fermi level, $E_{i}$ the energy of the $i$ th quantized level, $T$ the temperature and $k_{\mathrm{B}}$ the Boltzman constant. The calculated density is than plugged into Poisson equation (Eq. (3)) and the iteration repeated until convergence is achieved. Convergence of the selfconsistent algorithm can be improved adopting special relaxation techniques. Here we have used a first order expansion of the model reported in Ref. [56].

In the following, we consider two MODFET structures, namely a single heterojunction AlGaN/GaN NMODFET where the $\mathrm{AlGaN}$ donor layer is grown on 
top of the GaN channel layer, and an "inverted" GaN/ AlGaN/GaN MODFET (IMODHFET) where the channel layer is grown on top of the $\mathrm{AlGaN}$ donor layer. The N-MODFET structure consists (from the gate to the substrate) of a $150 \AA$ n-doped $\left(n=10^{18} \mathrm{~cm}^{-3}\right) \mathrm{Al}$ GaN, $50 \AA$ Anintentionally doped $\mathrm{AlGaN}$ layer and a thick GaN buffer. The IMODFET consists (from the gate to the substrate) in a $300 \AA$ unintentionally doped GaN layer, $50 \AA$ unintentionally doped AlGaN, $150 \AA$ n-doped $\left(n=10^{18} \mathrm{~cm}^{-3}\right)$ AlGaN, $300 \AA$ unintentionally doped AlGaN layer, and a thick GaN layer.

We consider a residual doping of $10^{17} \mathrm{~cm}^{-3}$ in both $\mathrm{GaN}$ and $\mathrm{AlGaN}$ layers. We use a Schottky barrier $\left(\phi_{\mathrm{B}}\right)$ of $1.1 \mathrm{eV}$ for the metal-GaN interface and a $\phi_{\mathrm{B}}=1.2 \mathrm{eV}$ for the metal-AlGaN interface. Calculations have been performed for $\mathrm{Al}_{x} \mathrm{Ga}_{1-x} \mathrm{~N}$ with $\mathrm{Al}$ concentrations of $x=0.1,0.2,0.3,0.4$. Both [0001] and [000-1] growth directions are considered. In the simulations, we have used an effective mass of $0.19 m_{0}$ for electrons and $1.8 m_{0}$ for holes in both $\mathrm{GaN}$ and $\mathrm{AlGaN}$ layers. The band gaps and band discontinuities of the AlGaN layers used are tabulated in Table 2.

As discussed previously the presence of polarization is quite important in the nitride-based N-MODFET. The conduction band edge profile for the N-MODFET grown in [0001] direction is depicted in Fig. 11 for the cases (i) with both spontaneous and PE polarization fields, (ii) without considering the polarization fields, (iii) with only the PE polarization fields. The difference in PE and spontaneous polarization between $\mathrm{AlGaN}$ and $\mathrm{GaN}$ layer manifest itself as a fixed 2D charge density at the interface between the two materials. For the [0001] growth direction considered in figure the difference in polarization between the two materials induces a positive charge $\left(\sigma=+1.12 \times 10^{13} \mathrm{~cm}^{-2}\right)$ at the $\mathrm{Al}_{0.2} \mathrm{Ga}_{0.8} \mathrm{~N} /$ $\mathrm{GaN}$ interface. Electrons are attracted by this positive charge, tending to accumulate at the interface and thus forming a conductive channel. Moreover, the high electric field due to the interface charge, favors the buildup of a large channel density and of a strong channel confinement. Within the AlGaN layer, the strong electric field compensates the space-charge contribution coming from the ionized donors. Consequently, it prevents the appearance of the parasitic channel that would otherwise form in the doped AlGaN layer [49,57].

Table 2

Band gap and conduction band discontinuities with respect to GaN of the $\mathrm{Al}_{x} \mathrm{Ga}_{1-x} \mathrm{~N}$ layer

\begin{tabular}{lll}
\hline$x(\mathrm{Al})$ & $E_{\mathrm{G}}(\mathrm{eV})$ & $\Delta E_{\mathrm{C}}(\mathrm{eV})$ \\
\hline 0.1 & 3.62 & 0.17 \\
0.2 & 3.85 & 0.33 \\
0.3 & 4.09 & 0.51 \\
0.4 & 4.35 & 0.69 \\
\hline
\end{tabular}

The comparison reported in Fig. 11 between the three cases with different contribution of the polarization fields shows the importance of considering both spontaneous and PE polarizations in GaN-based device modeling. In fact, neglecting the spontaneous polarization, as was done recently [58-61], the channel electron density is underestimated [49]. Clearly the sign of the polarization charge is crucial. For the same N-MODFET structure grown in the [000-1] direction, the resulting polarization charge would be negative (with the same magnitude) and electrons would be repelled from the channel as shown in Fig. 12.

The distribution of the free electron charge in the channel is shown in Fig. 13 for several values of the Al concentration of the $\mathrm{AlGaN}$ layer. Increasing the $\mathrm{Al}$ content induces a larger polarization charge at the GaN/ $\mathrm{AlGaN}$ interface and consequently a higher channel electron concentration.

The results of calculations we have shown so far are obtained by considering only the polarization charge at

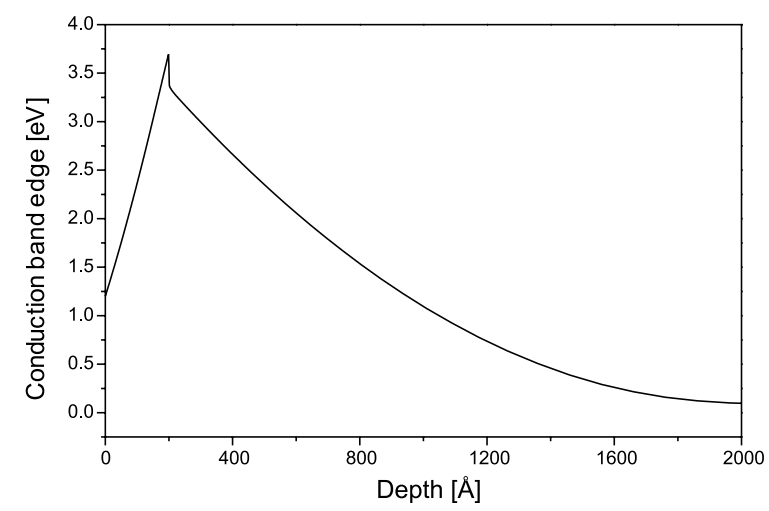

Fig. 12. Conduction band edge for the N-MODFET structure grown in the [000-1] direction for $V_{\mathrm{G}}=0$.

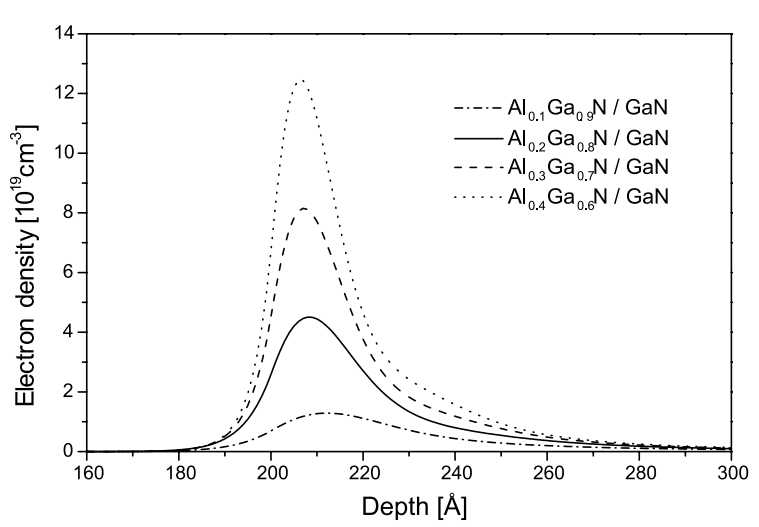

Fig. 13. Electron density distribution in the channel of the [0001] grown N-MODFET for $V_{\mathrm{G}}=0$ for several Al contents of the $\mathrm{Al}_{x} \mathrm{Ga}_{1-x} \mathrm{~N}$ layer. 
the $\mathrm{AlGaN} / \mathrm{GaN}$ interface. In reality, however, polarization charges that form at the metal- $\mathrm{AlGaN}$ and at the end of the GaN buffer region should also be accounted for. The metal-AlGaN charge is completely screened by the charges induced on the metal surface and can therefore be neglected. On the other hand, the charges at the end of the buffer region may induce large deviation with respect to the situation described above. Oberhuber et al. [49] have considered a $-\sigma / 2$ charge at the interface between $\mathrm{GaN}$ and a nucleation region. The exact amount of such charge depends however on the morphology of the heterojunction and may differ from the theoretical value $\sigma=\Delta P / e$. The situation is less critical if the bottom interface is far away from the main $\mathrm{AlGaN} / \mathrm{GaN}$ heterojunction. In this case, the polarization charge that arises can be completely screened by the residual doping of the GaN substrate. On the contrary, if such interface is close to the $\mathrm{AlGaN} / \mathrm{GaN}$ heterojunction, the polarization charge can completely deplete the channel. In our simulations we have considered a thick GaN substrate. Thus, the effect of the polarization charge at the end of the GaN substrate is completely screened.

The band edge profile and electron densities for the IMODFET grown in the [000-1] direction are shown in Figs. 14 and 15 respectively. A comparison of the conduction band edges with and without polarization charges is also plotted. As for the N-MODFET, the presence of the fixed and positive polarization charge at the GaN/AlGaN interface induces the formation of a channel not present in absence of the polarization charge. For the I-MODFET a $-\sigma$ polarization charge is also present at the end of $\mathrm{AlGaN}$ region (i.e., at the $\mathrm{AlGaN} / \mathrm{GaN}$ interface). Similar to the [0001] grown NMODFET, a larger $\mathrm{Al}$ content of the AlGaN layer induces a larger polarization charge at the GaN/AlGaN interface and consequently an increase of electron con-

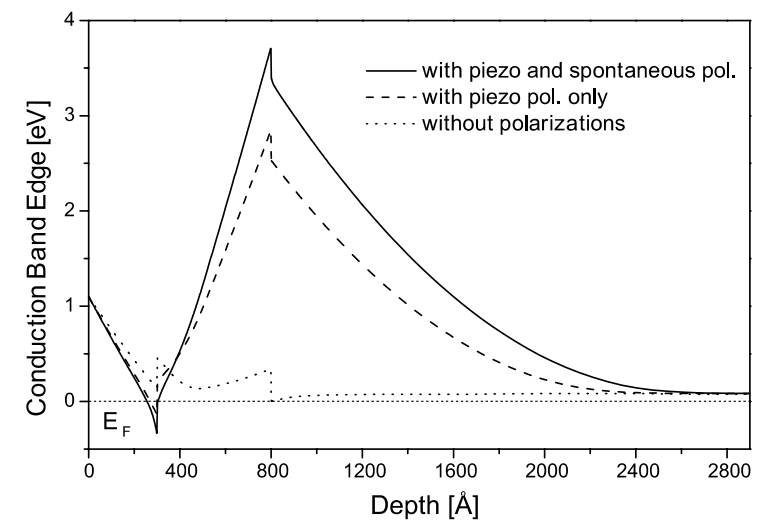

Fig. 14. Conduction band edge for the I-MODFET structure grown in the $[000-1]$ direction for $V_{\mathrm{G}}=0$ with and without polarization fields.

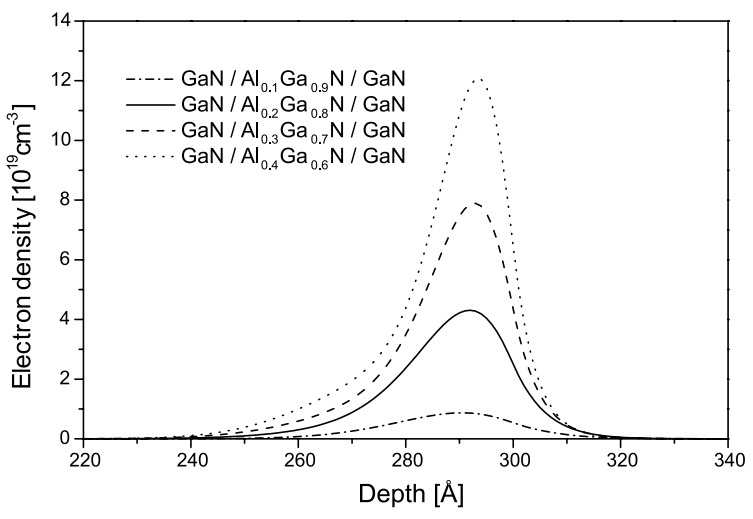

Fig. 15. Electron density distribution in the channel of the $[000-1]$ grown I-MODFET for $V_{\mathrm{G}}=0$ for several $\mathrm{Al}$ contents of the $\mathrm{Al}_{x} \mathrm{Ga}_{1-x} \mathrm{~N}$ layer.

centration in the channel. Naturally, for [0001] orientation the interface charge forms below the $\mathrm{AlGaN}$ layer which is not what is desired for an I-MODFET. What is desired is the formation of the electron sheet layer on top of the AlGaN layer, which is possible when the $[000-1]$ orientation is employed. The structure in its present shape, i.e., the [0001] polarity would show FET performance providing that the $\mathrm{AlGaN}$ layer is completely depleted but with small transconductance. If the $\mathrm{AlGaN}$ is not depleted, then the device would function as metal semiconductor FET (MESFET) dominated by transport in the $\mathrm{AlGaN}$ layer unless the gate potential is large enough to deplete the $\mathrm{AlGaN}$ layer. To eliminate the formation of an interface electron charge at the bottom of the AlGaN layer, the bottom heterointerface should be graded substantially. In that case, the [0001] polarity would cause the band diagram to accumulate holes at the top interface if they are present. That top interface would accumulate electrons in the [000-1] polarity.

The channel charge density is therefore controlled by two factors: (i) the gate bias as in traditional N-MODFET device, (ii) the $\mathrm{Al}$ content of the $\mathrm{AlGaN}$ layer, which tailors the polarization field. Charge control in nitride-based devices can be achieved by adjusting two independent parameters and thus offers a wide degree of flexibility with respect to traditional devices. This can be seen from the sheet charge concentration in the channel as obtained by integrating the electron density distribution along the $z$-direction. Considering the explicit dependence of the sheet charge density on the gate voltage $V_{\mathrm{G}}$, we have:

$n_{\mathrm{s}}\left(V_{\mathrm{G}}\right)=\int n\left(V_{\mathrm{G}}, z\right) \mathrm{d} z$

Figs. 16 and 17 show the sheet electron density in the channel as a function of gate bias for several $\mathrm{Al}$ contents 


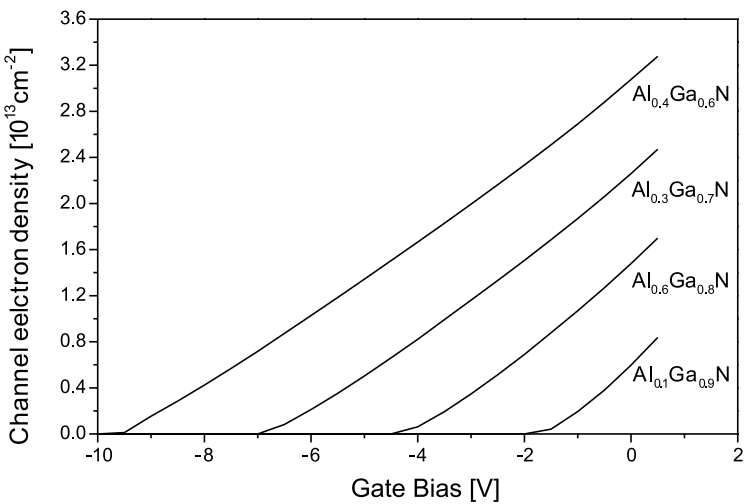

Fig. 16. Channel electron density as a function of $\mathrm{Al}$ and $V_{\mathrm{G}}$ for the [0001] grown N-MODFET.

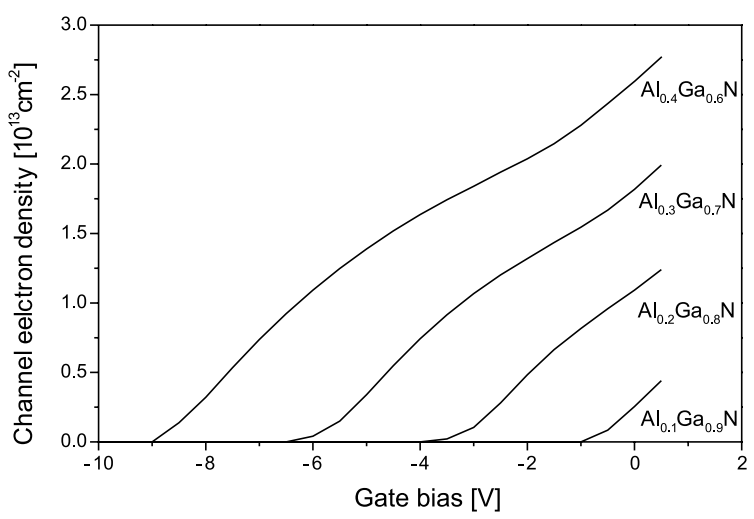

Fig. 17. Channel electron density as a function of $\mathrm{Al}$ and $V_{\mathrm{G}}$ for the [000-1] grown I-MODFET.

in the AlGaN layer for both [0001] grown N-MODFET and [000-1] grown I-MODFET, respectively. Naturally, the channel electron density increases for larger $\mathrm{Al}$ contents of the AlGaN layer. We note also that the density is higher for the N-MODFET with respect to the I-MODFET because the particulars relating to the band bending on the top interface of the I-MODFET. As mentioned earlier, the I-MODFET structure is intended to be used with [000-1] for investigative purposes only as the body of work in the $\mathrm{AlGaAs} / \mathrm{GaAs}$ system showed the N-MODFET to the desired device structure.

\section{Drain current model in MODFETs}

We have implemented a quasi-2D [62-64] model for the calculation of the current-voltage characteristics of the nitride MODFETs. This model makes use of the exact value of the sheet charge density in a MODFET device channel, obtained from the self-consistent Schrödinger-Poisson solution presented above.

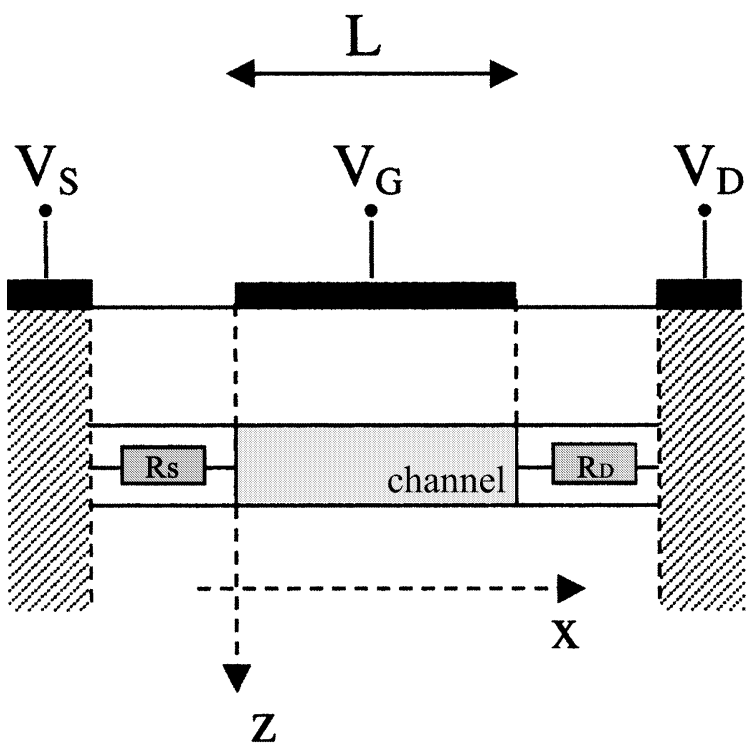

Fig. 18. Schematic representation of the quasi-2D FET model used.

We assume a FET model shown in Fig. 18 where the $x$-axis is along the channel and the $z$-axis is along the growth direction. The model also considers the presence of a drain $\left(R_{\mathrm{D}}\right)$ and source $\left(R_{\mathrm{S}}\right)$ resistance. When a drain bias $\left(V_{\mathrm{D}}\right)$ is applied, the potential along the channel may be considered as varying gradually from the source bias $V_{\mathrm{S}}$ to $V_{\mathrm{D}}$. In this situation it is still possible to calculate the sheet charge density $n_{\mathrm{s}}$ at every section grid, provided that one considers the $V(x)$ potential (on the top surface) for each point of the channel. Since for $\mathrm{n}$-channel devices, $V_{\mathrm{D}}$ is positive and $V_{\mathrm{S}}$ is zero, $V(x)$ contributes to the channel depletion and the sheet charge density $n_{\mathrm{s}}$ for the generic $x$ section of the FET will be therefore:

$n_{\mathrm{s}}(x)=n_{\mathrm{s}}\left(V_{\mathrm{G}}-V(x)\right)$

By neglecting diffusion contributions, the source-todrain current $I_{\mathrm{DS}}$ is given by:

$I_{\mathrm{DS}}=-q W v(x) n_{\mathrm{s}}(x)$

where $W$ is the gate width and $v(x)$ the electron mean velocity, supposed independent of the transverse coordinate. The dependence of the drift velocity on the longitudinal electric field is empirically given by:

$v(x)=\frac{\mu_{0} F(x)}{1+\frac{F(x)}{F_{\mathrm{C}}}}$

where $F(x)$ is the electric field $(=-\mathrm{d} V(x) / \mathrm{d} x), \mu_{0}$ is low field mobility and $F_{\mathrm{C}}=v_{\text {sat }} / \mu_{0}$ is the electric field at saturation. Parasitic components are included explicitly through the drain and source resistances $\left(R_{\mathrm{D}}, R_{\mathrm{S}}\right)$ 
$V_{\mathrm{S}}^{\mathrm{e}}=V_{\mathrm{S}}+I_{\mathrm{DS}} R_{\mathrm{S}}$

$V_{\mathrm{D}}^{\mathrm{e}}=V_{\mathrm{D}}-I_{\mathrm{DS}} R_{\mathrm{D}}$

where $V_{\mathrm{D}}^{\mathrm{e}}$ and $V_{\mathrm{S}}^{\mathrm{e}}$ represent the effective bias boundaries of the gate region on the drain and source sides, respectively. For a certain value of $I_{\mathrm{DS}}$, we can calculate $V_{\mathrm{D}}$ by solving Eq. (7). The explicit equation for the current is:

$I_{\mathrm{DS}}=q W \frac{\mu_{0} F(x)}{1+\frac{F(x)}{F_{\mathrm{C}}}} n\left(V_{\mathrm{G}}-V(x)\right)$

The numerical solution is based on the discretization of this expression into $N$ sections, each with amplitude $h$, so that $N h=L$, where $L$ is the gate length. Given the $(i-1)$ th section potential, the $i$ th potential $V_{i}=V_{i-1}+$ $F_{i} h$, where $F_{i}$ is the $i$ th section electric field. We have then the $N$ relations:

$I_{\mathrm{DS}}=q W \frac{\mu_{0} F_{i}}{1+\frac{F_{i}}{F_{\mathrm{C}}}} n\left(V_{\mathrm{G}}-V_{i-1}-F_{i} h\right)$

since the $(i-1)$ th section potential is known from the previous step, this is a non-linear equation in the unknown $F_{i}$. Solving iteratively for all the $N$ sections, one obtains the value of the drain voltage $V_{\mathrm{D}}$ consistent with the assumed current.

Repeating this procedure for a suitable range of values of $I_{\mathrm{DS}}$, one obtains the set of corresponding values of $V_{\mathrm{DS}}$ and thus the MODFET $I-V$ characteristics, which are elaborated on below.

\section{I-V characteristics}

In this section we discuss the simulated $I-V$ characteristic of the normal and inverted MODFET, obtained for a gate length of $L=0.3 \mu \mathrm{m}$. We have chosen a drain and source contact resistivity of about $1 \Omega \mathrm{mm}$ which is consistent with experimentally measured values on these types of devices [65]. We use a saturation velocity of $2.5 \times 10^{7} \mathrm{~cm} / \mathrm{s}$, while for the low field mobility we choose a value of $\mu_{0}=1100 \mathrm{~cm}^{2} \mathrm{~V}^{-1} \mathrm{~s}^{-1}$, slighter higher than the GaN bulk value, according to the experimental and theoretical results for similar devices [22,49,66,67].

In Fig. 19, we show the $I_{\mathrm{DS}}$ vs. $V_{\mathrm{DS}}$ for the [0001] polarity MODFET for several gate $\left(V_{\mathrm{GS}}\right)$ voltages. The results are presented for both $x=0.2$ (Fig. 19a) and $x=0.4$ (Fig. 19b), Al concentration of the top layer. For $x=0.2$, the MODFET reaches pinch-off for a bias voltage of $V_{\mathrm{GS}}=-4.4 \mathrm{~V}$ while for $x=0.4$ the pinch-off is reached at $V_{\mathrm{GS}}=-9.5 \mathrm{~V}$. On the other hand, the saturation drain current for $x=0.2$ is $I_{\mathrm{DS}}=2.4 \mathrm{~A} / \mathrm{mm}$ at $V_{\mathrm{GS}}=0$ and it increase up to $5.76 \mathrm{~A} / \mathrm{mm}$ for an $\mathrm{Al}$ content of $x=0.4$. Thus, the current flowing in the devices depends strongly on the $\mathrm{Al}$ contents of the top layer. This is essentially due to increasing of the channel
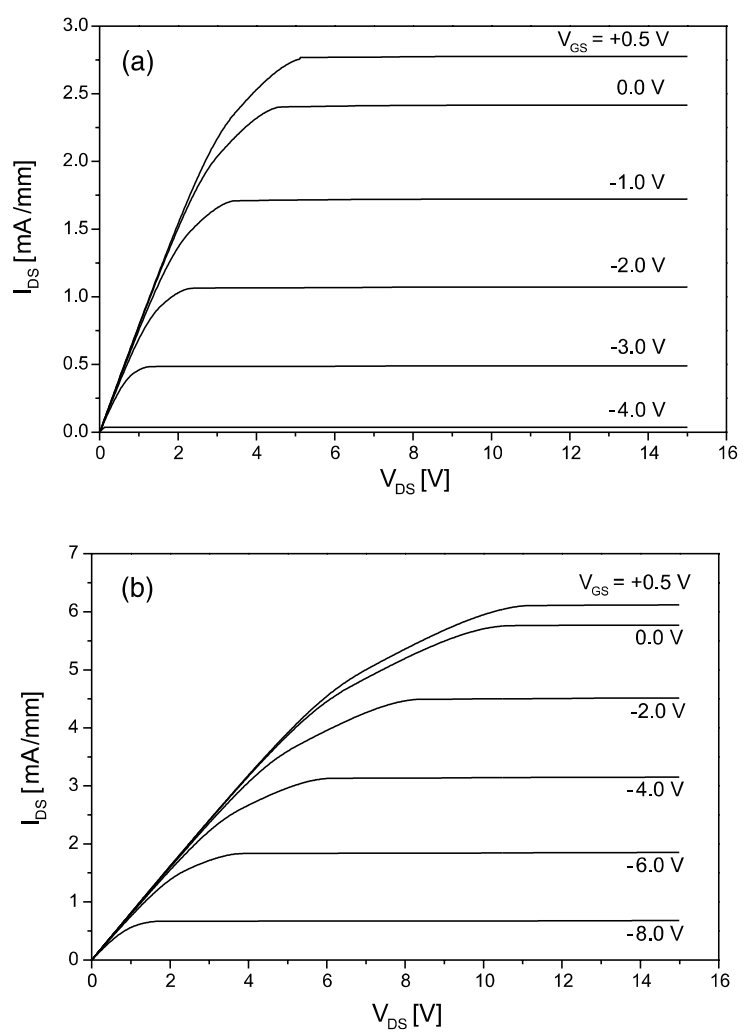

Fig. 19. (a) $I_{\mathrm{DS}}$ vs. $V_{\mathrm{DS}}$ for the [0001] polarity N-MODFET for several gate voltages $\left(V_{\mathrm{GS}}\right)$ and for $x=0.2$. (b) $I_{\mathrm{DS}}$ vs. $V_{\mathrm{DS}}$ for the [0001] polarity N-MODFET for several gate voltages $\left(V_{\mathrm{GS}}\right)$ and for $x=0.4$.

electron density induced by the increasing in the polarization charge going from an $\mathrm{Al}$ due to increase in content of $0.2-0.4$. This peculiarity of the MODFET should be considered in the design of these devices since fluctuation of the alloy composition of the top layer may induce large variation with respect to nominal electrical values of the device. It should also be pointed out that the gate leakage would determine the extent of gate voltage that can be applied to the gate. For a gate bias of $9.5 \mathrm{~V}$ and $\mathrm{AlGaN}$ layer thickness of $20 \mathrm{~nm}$, the vertical field under the gate near the source can reach 4.75 MV/ $\mathrm{cm}$. This means that MODFETs utilizing large mole fractions of $\mathrm{Al}$ may require thin $\mathrm{AlGaN}$ layers or recessed gates to keep the gate voltage smaller.

A similar situation is obtained for the I-MODFET with the GaN/AlGaN/GaN structure grown in the [000-1] direction, meaning with $\mathrm{N}$ polarity. The calculated $I_{\mathrm{DS}}$ vs. $V_{\mathrm{DS}}$ characteristics are reported in Fig. 20a and $\mathrm{b}$ for $x=0.2$ and $0.4 \mathrm{Al}$ compositions of the AlGaN layer, respectively. Also in this case the pinch-off bias depends critically on the Al composition and varies from $-3.9 \mathrm{~V}$ for $x=0.2$ to $-9.0 \mathrm{~V}$ for $x=0.4$. Saturation 

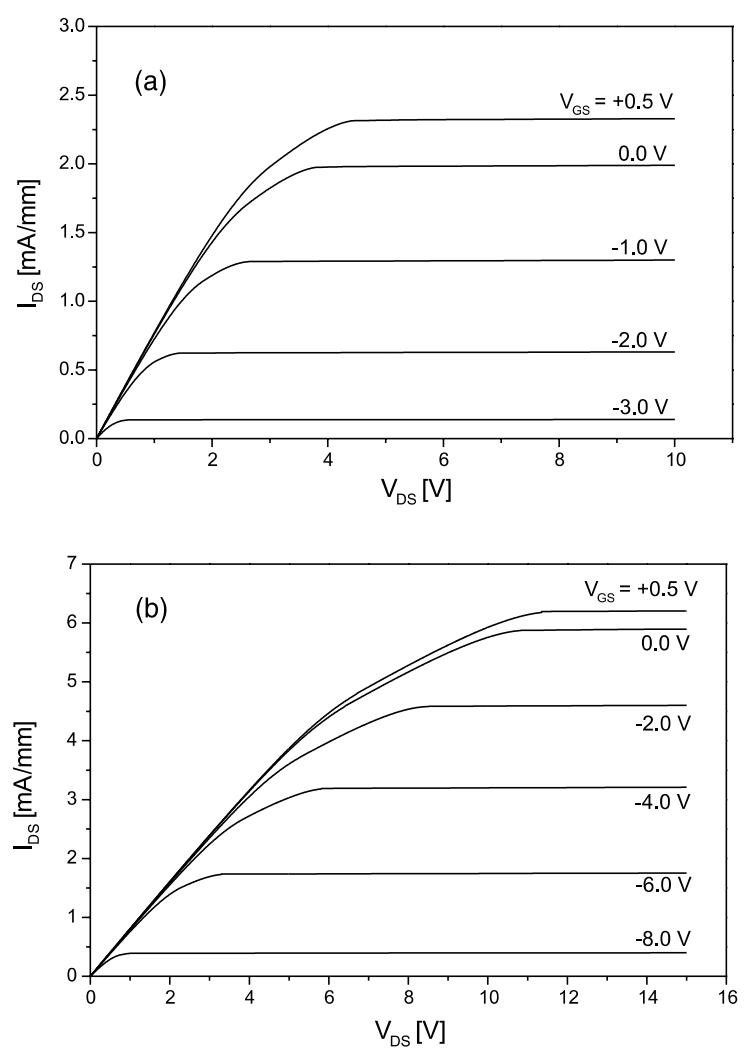

Fig. 20. (a) $I_{\mathrm{DS}}$ vs. $V_{\mathrm{DS}}$ for the $[000-1]$ polarity Inverted MODFET for several gate voltages $\left(V_{\mathrm{GS}}\right)$ and for $x=0.2$. (b) $I_{\mathrm{DS}}$ vs. $V_{\mathrm{DS}}$ for the $[000-1]$ polarity inverted MODFET for several gate voltages $\left(V_{\mathrm{GS}}\right)$ and for $x=0.4$.

currents are lower for the I-MODFET at $x=0.2$ with respect to the equivalent MODFET structure. Such difference, however, is negligible for the case with $x=0.4$.

\section{Experimental considerations}

GaN-based FETs are intended primarily for power applications at high frequencies. Consequently, traditional small signal considerations have to be augmented by large signal specific issues. The main parameter facing a power device is the maximum power level that can be obtained and the associated gain. In many applications the noise figure of the device must also be considered. In simple terms if the device has a large drain breakdown voltage, high gain at high frequencies, and high drain efficiency, the stage is set for a desirable device. Even in a well-designed device in a semiconductor with all the accolades, the thermal wall is a very formidable one. Thus, it is imperative that the effect of temperature on device performance is accounted for accurately. As in

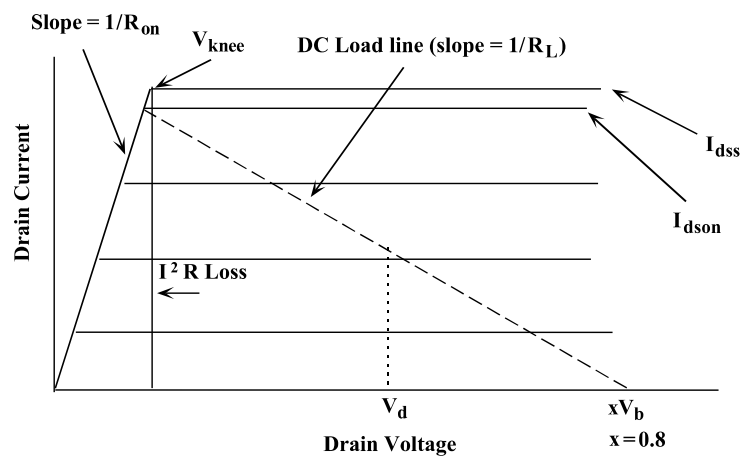

Fig. 21. Schematic representation of $I-V$ characteristics with a load line for a Class-A operation. $V_{\mathrm{b}}$ is the breakdown voltage.

small signal modeling, the first step in power modeling is to establish the basic device geometrical factors that are needed to calculate the current-voltage characteristics. Once these are known, the output characteristics superimposed with the load line can be used to estimate the power level that can be obtained from the device provided that it is not limited by the input drive as shown in Fig. 21. In Class-A operation, the maximum power that can be expected from the drain circuit of a device is given by

$P_{\max }=\frac{I_{\mathrm{d} \text { son }}\left(V_{\mathrm{b}}-V_{\text {knee }}\right)}{8}$

where $I_{\mathrm{d} \text { son }}$ is the maximum drain current (this is the drain current with a small positive voltage on the gate electrode), $V_{\mathrm{b}}$ is the drain breakdown voltage, and $V_{\mathrm{knee}}$ is the knee voltage as shown in Fig. 21. The allowable positive gate voltage $(\approx 1 \mathrm{~V})$ will depend on the channel doping and the work function of the gate metal. The positive gate voltage is limited by the onset of forward Schottky-diode current. The DC load line shown in Fig. 21 would be used in a Class-A RF amplifier with the maximum drain voltage $V_{\mathrm{d}}=V_{\mathrm{b}} / 2$. The slope of the load line is $1 / R_{\mathrm{L}}$ where $R_{\mathrm{L}}$ is the value of the load resistance at the output of the FET. What can be gleaned from Eq. (12) is that $V_{\mathrm{b}}$ and $I_{\mathrm{d} \text { son }}$ must be made as large as possible. The utility of wide band gap semiconductors such as $\mathrm{GaN}$ at this juncture is that the drain breakdown voltage is larger than that in conventional Group III-V semiconductors. In general, the drain can be swung to voltages up to within $80 \%$ of the drain breakdown for a $20 \%$ margin of safety. It should be pointed out that the maximum drain current in nitride semiconductor-based MODFETs is in the same ballpark as that for more conventional semiconductors. This implies that increased power handling capability is a direct result of large breakdown voltages and thermal conductivity and the fact that higher junction temperatures can be tolerated. 
Ability to increase drain bias increases the load resistance and makes it easier to impedance match, particularly in devices with large gate widths.

In power devices, power dissipation within the device increases the junction temperature and alters the output characteristics. On the one hand, higher junction temperatures with respect to the case temperature would enhance the heat dissipation to the power of four of the temperature differential, but along with it come reduced current and increased series resistances, which in turn increase the heat dissipation. Moreover, the thermal conductivity of the semiconductor decreases with increased temperature, exacerbating the situation. Temperature-dependent material parameters, if known, can be used to calculate the output characteristics with respect to temperature. However, a more pragmatic approach, particularly when the aforementioned parameters and, or models required are not available, can be taken in which one measures the output characteristics of the device under consideration as a function of temperature. The junction temperature is critically dependent on the substrate thermal conductivity that is available for various substrates including GaN [1]. The functional dependence of thermal conductivity on temperature is

$\chi(T)=\chi\left(T_{0}\right)\left(T / T_{0}^{-r}\right)$

where the coefficient $r$ is $0.559,0.443,0.524$, and 0.544 for $\mathrm{Si}, \mathrm{GaAs}, \mathrm{SiC}$, and sapphire, respectively [68]. Thermal conductivities of sapphire, $\mathrm{SiC}, \mathrm{GaAs}$, and $\mathrm{Si}$ as a function of temperature are shown in Fig. 22. In Fig. 22, $\chi\left(T_{0}\right)$ has also been appropriately reduced to account for the doping of the substrate material.

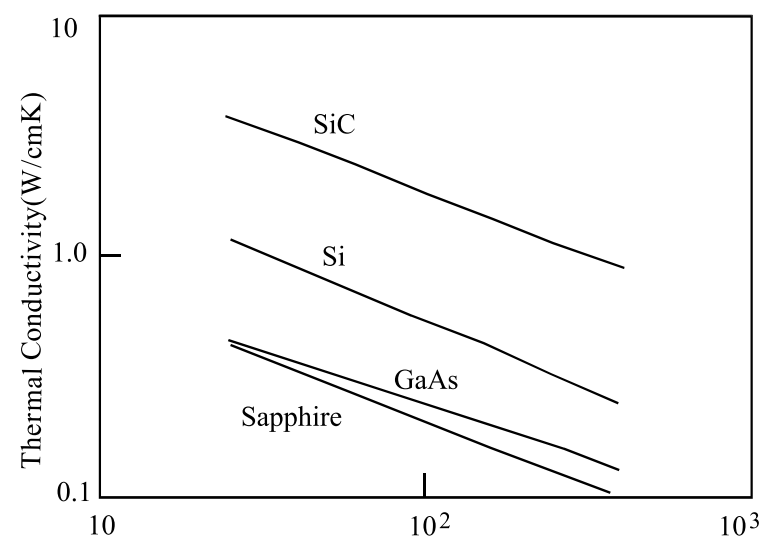

Temperature $\left({ }^{\circ} \mathrm{C}\right)$

Fig. 22. Thermal conductivity vs. temperature for $\mathrm{SiC}$ and sapphire (after Ref. [66]).

\section{Schottky barriers for gates}

Any semiconductor device requires metal contacts and MODFETs are no exception. These devices require ohmic source and drain contacts as well as a rectifying Schottky barrier for controlling the charge in the channel. Schottky barrier-related processes for GaN-based devices are nascent, but rapid progress is being made. Until recently it has been difficult to fabricate good quality single-crystal films on which a Schottky metal could be deposited, and upon which the properties of Schottky barriers could be studied. However, considerable progress has been made with Pt-GaN Schottky barriers $[69,70]$ that have been successfully implemented in GaN-based MODFETs [24,71,72].

Recent successes in growing good quality singlecrystal GaN layers prompted the fundamental electrical property studies of metal-semiconductor barriers on $\mathrm{GaN}$. In order to determine the properties of only the metal-semiconductor junction, one must be able to model the semiconductor. Semiconductors with large defect concentrations are notorious for exhibiting parasitic processes in current-voltage and capacitance-voltage characteristics that cloud the picture. Consequently, good epitaxial layers as well as good metal-semiconductor interfaces are imperative. During the evolutionary period, while the sample quality is acceptable, temperature and frequency dependence of the capacitance-voltage characteristics and temperature-dependent current-voltage characteristics are measured and analyzed for determining effective metal-semiconductor barrier height. To get a large Schottky barrier height for rectifying metal contacts on $\mathrm{GaN}$, which is imperative for low leakage, metals with large work functions such as Au and Pt [69] have been explored. Hacke et al. [73] have studied Schottky barriers made of Au on unintentionally doped $\mathrm{n}-\mathrm{GaN}$ grown by HVPE. The forward current ideality factor was $n_{\mathrm{idl}} \sim 1.03$ and the reverse bias leakage current was $<10^{-10} \mathrm{~A}$ at a reverse bias of $-10 \mathrm{~V}$. While the current-voltage measurement indicated the barrier height to be $0.844 \mathrm{eV}$, the capacitance measurements led to a value of $0.94 \mathrm{eV}$.

Suzue et al. [69], and Mohammad et al. [70] have studied the Pt Schottky barriers on unintentionally doped n-GaN. Temperature-dependent current-voltage and capacitance-voltage characteristics in the range of $-195-42{ }^{\circ} \mathrm{C}$ were studied to gain insight about the current conduction mechanism. Any excess current observed is traditionally attributed to defect (generation-recombination $(\mathrm{G}-\mathrm{R})$ centers) and surface leakage current. The ensuing current is called the ShockleyRead-Hall (SRH) recombination current resulting from the mid-gap states. If one neglects this excess current, a barrier height of about $0.8 \mathrm{eV}$ is deduced as opposed to about $1 \mathrm{eV}$ deduced from the $C V$ measurements. Because of the effect of excess current on the slope of the 
$I-V$ curve, $C-V$ measurements in this particular case may represent the metal barrier height. An examination of the $C-V$ plots, however, indicated that under reverse bias condition, the capacitance depended insignificantly on the sample temperature and the signal frequency as shown in Fig. 23a and b. This leads one to conclude that the density of traps has been lowered. The curves corresponding to all temperatures were largely linear, which yielded barrier heights ranging between 0.95 and 1.05 $\mathrm{eV}$. Reduced capacitance with decreasing temperature is consistent with relatively deep donors. Binari et al. [74] determined Ti Schottky barrier heights to be 0.58 and
$0.59 \mathrm{eV}$ from the current-voltage and capacitance measurements, respectively. The ideality factor $n_{\text {idl }}$ was approximately 1.28 . The diode series resistance $\left(R_{\mathrm{s}}\right)$ was $100 \Omega$.

The ternary $\mathrm{Al}_{x} \mathrm{Ga}_{1-x} \mathrm{~N}$ is an essential component of nitride-based MODFETs, which makes the investigation of metal $\mathrm{Al}_{x} \mathrm{Ga}_{1-x} \mathrm{~N}$ contacts imperative. Khan et al. [75] reported the fabrication of a $\mathrm{Cr} / \mathrm{Au}$ Schottky barrier on n-AlGaN. Moreover, Khan et al. [76] studied the Schottky barrier characteristics of the $\mathrm{Au}-\mathrm{Al}_{x} \mathrm{Ga}_{1-x} \mathrm{~N}$ system. A typical current-voltage characteristic of an $\mathrm{Al}_{0.14} \mathrm{Ga}_{0.86} \mathrm{~N}$ Schottky diode had an ideality factor of
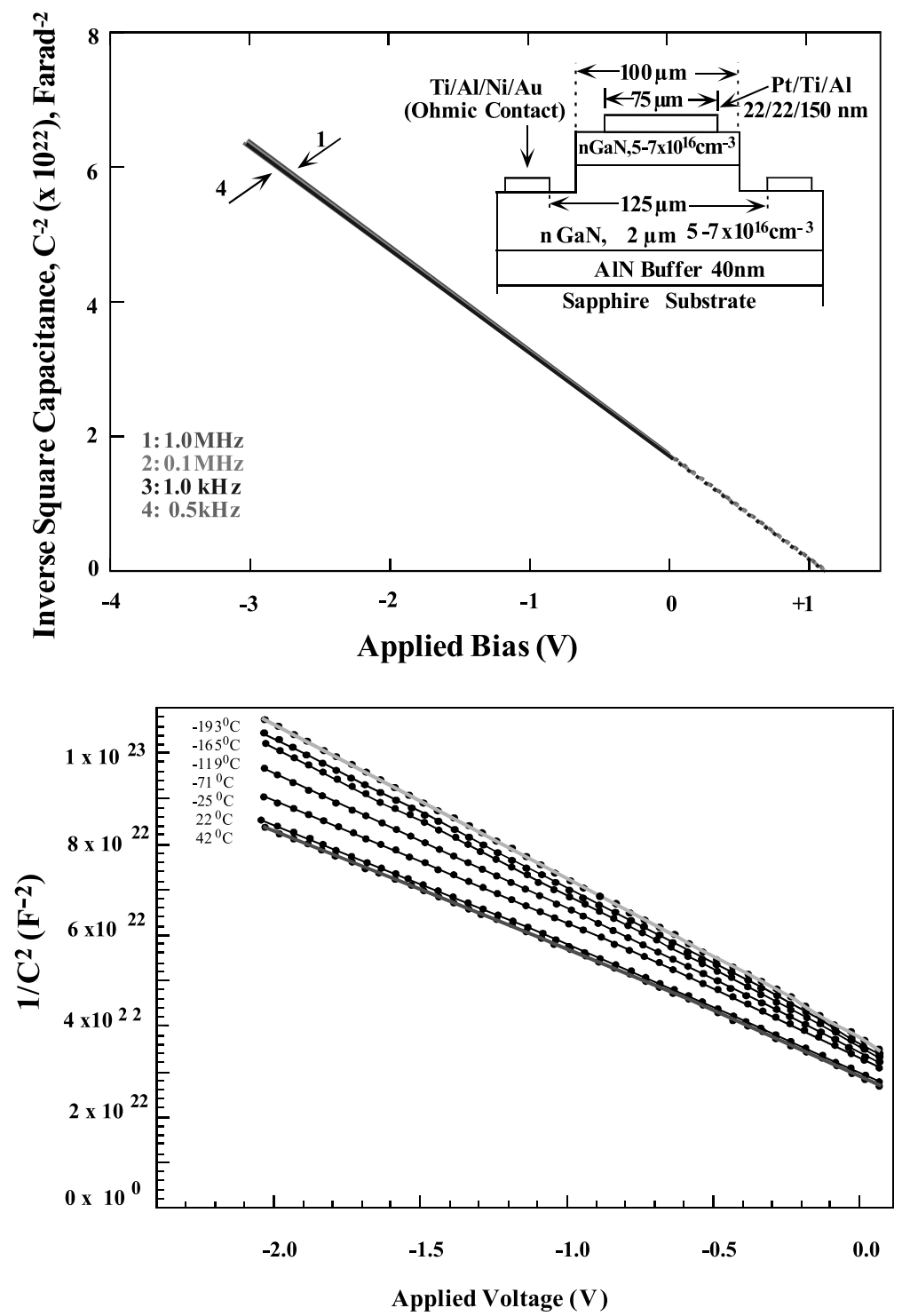

Fig. 23. (a) Variation of inverse square capacitance, $C^{-2}$ vs. applied bias $V$ for various signal frequencies in Pt-GaN Schottky barriers. Curves 2 and 3 lie in between curves 1 and 4, respectively. (b) Temperature dependence of the $C^{-2}$ vs. applied bias $V$ in Pt-GaN Schottky barriers. 
1.56 under reverse bias and a threshold voltage of about $0.9 \mathrm{~V}$ at $0.1 \mathrm{~A}$. The reverse bias leakage current was recorded to be marginally low $\left(10^{-10} \mathrm{~A}\right)$ for a reverse bias of $-10 \mathrm{~V}$. By using the current-voltage method, the barrier height and the electron affinity were determined to be 0.94 and $4.16 \mathrm{eV}$, respectively. From the $C^{-2}$ vs. $V$ plot, the same barrier height and the electron affinity were deduced to be $1.3 \pm 0.05$ and $3.8 \mathrm{eV}$, respectively. As the AlGaN quality increases, periodic as well as more in-depth investigations will become necessary to get an accurate picture of intrinsic parameters. In short, the current conduction mechanism in metal-semiconductor structures is strongly affected by surface and bulk states. Deviations from an ideal ideality factor, as is the case here, indicate such states. The situation gets more complicated with $\mathrm{AlGaN}$ and gets worse as the AlN mole fraction is increased. Likewise, capacitancevoltage measurements also are affected by states that are charged, either by interface states or by bulk states. As is the case in many facets of research and development, insights into the metal-nitride contacts will be gained in an evolutionary manner hinging upon the developments in nitride layers.

\section{Contacts to GaN}

Ohmic contacts in power devices are extremely important because they affect their efficiency as well as heat dissipation. Initial inferior results helped fuel concerns that GaN-based electronic devices may not perform well. Early specific contact resistivities on n-type $\mathrm{GaN}$ using $\mathrm{Al}$ and $\mathrm{Au}$ metallizations [77] were in the range of $10^{-4}$ and $10^{-3} \Omega \mathrm{cm}^{2}$. Major improvements were realized by using $\mathrm{Ti} / \mathrm{Au}$ [78] and $\mathrm{TiAl}$ [79], in that specific contact resistivities in the high $10^{-6} \Omega \mathrm{cm}^{2}$ were obtained with the latter. Carrying the TiAl contact work one step further, Wu et al. [80] confirmed that, except at very high annealing temperatures, the ohmic contact suggested by Lin et al. [79] functions very effectively. At very high temperatures, $\mathrm{Al}$ of the metal contact melts and tends to ball up, resulting in rough surfaces and increased ohmic contact resistances as pointed out already by Lin et al. [79]. In an attempt to circumvent this difficulty, Wu et al. [80] designed a separate layer-metallization method where a realignment and deposition of a second thin $\mathrm{Ti}$ layer, and a $2000 \AA \mathrm{Au}$ overlayer were carried out. Specific contact resistivities were in the range of $3.0 \times$ $10^{-6}-5.5 \times 10^{-6} \Omega \mathrm{cm}^{2}$, depending on the doping concentration in the semiconductor.

In an attempt to obtain improved ohmic contacts, Fan et al. [81] have designed a multilayer ohmic contact method. By using a composite metal layer of Ti/Al/Ni/ Au (150/2200/400/500 §), they obtained very low contact resistivities. Specifically, for $\mathrm{n}-\mathrm{GaN}$ with doping levels between $2 \times 10^{17}$ and $4 \times 10^{17} \mathrm{~cm}^{-3}$, the specific

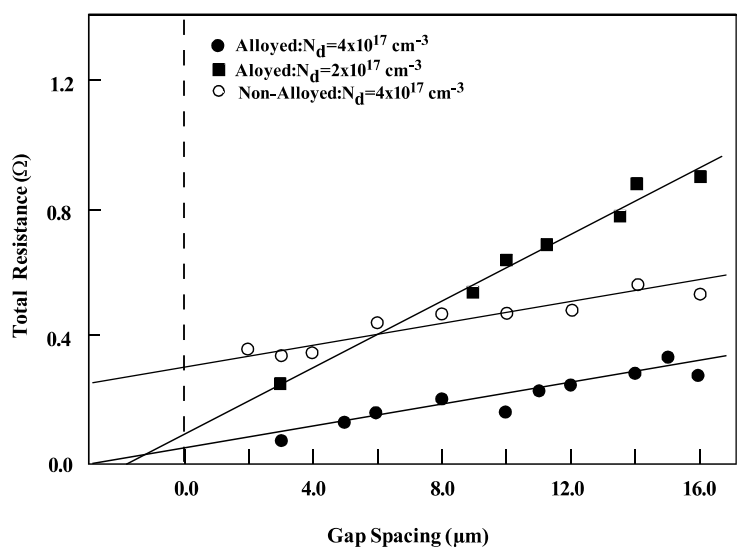

Fig. 24. Least-squares linear regression of the total resistance between the two adjacent ohmic contact pads in multiple-layer $\mathrm{Ti} / \mathrm{Al} / \mathrm{Ni} / \mathrm{Au}$ ohmic contacts on $\mathrm{GaN}$.

contact resistivities in the range of $\rho_{\mathrm{s}}=1.19 \times 10^{-7}$ and $8.9 \times 10^{-8} \Omega \mathrm{cm}^{2}$, respectively, were obtained. The resistance $R_{\mathrm{T}}$ between the two contacts was measured at $300 \mathrm{~K}$ using a four-point probe arrangement. The contact resistivity $\rho_{\mathrm{s}}$ was derived from a plot of $R_{\mathrm{T}}$ vs. gap length. The method of least squares was used to fit a straight line to the experimental data. These straight lines, and the actual experimental results for both the alloyed and non-alloyed contacts are shown in Fig. 24. Calculation of the contact resistivity was based on the assumption that the semiconductor sheet resistance underneath the contacts remained unchanged, which is not true for non-alloyed contacts. As for the current conduction mechanism in these ohmic contacts, the large metal-semiconductor barriers diminish the possibility of thermionic-emission-governed ohmic contacts to GaN. The alternative mechanism is some form of tunneling that may take place if $\mathrm{GaN}$ is so heavily doped as to cause a very thin depletion region. Tunneling is possible if, due to annealing (for example, at $900{ }^{\circ} \mathrm{C}$ for $30 \mathrm{~s}$ ), $\mathrm{Al}$ and $\mathrm{Ti}$ along with $\mathrm{Ni}$ undergo substantial interaction with each other and $\mathrm{GaN}$. Investigations showed that $\mathrm{Ti}$ receives $\mathrm{N}$ from $\mathrm{GaN}$, forming a metallic layer, while the lack of $\mathrm{N}$ on GaN provides the desired benefit of increased electron concentration through $\mathrm{N}$ vacancy formation [82]. Aluminum passivates the surface and also possibly reacts with Ti to form TiAl.

\section{AlGaN/GaN MODFETs}

To reiterate, MODFET's performance is due to the conduction channel that allows large sheet carrier concentrations to be maintained and its unique capacitance-voltage relationship [83]. Moreover, spatial separation of scattering centers (such as ionized donors) 
from the electrons leads to low field transport void of ionized impurity scattering. As discussed in detail above, what is somewhat unique to $\mathrm{GaN}$ and its alloys is the spontaneous and the strain-induced PE polarization that causes redistribution of mobile and weakly bound charge and charge collected from metal contacts.

\section{Experimental performance of GaN MODFETs}

Initial GaN MODFETs utilized the background donors in the $\mathrm{AlGaN}$ layer, the density of which is not controllable, to say the least, and any other free and weakly bound electrons drawn to the interface. Congruent with the early stages of development and the defect-laden nature of the early $\mathrm{GaN}$ and $\mathrm{Al}_{x} \mathrm{Ga}_{1-x} \mathrm{~N}$ layers, the MODFETs exhibited very low transconductances (on the order of $20 \mathrm{mS} / \mathrm{mm}$ ), and large on resistances. In addition, they also exhibited a low-resistance state, which was relatively high to begin with, and a high-resistance state before and after the application of a high drain voltage $(20 \mathrm{~V})$. As in the case of GaAs/ AlGaAs MODFETs, hot electron trapping in the larger band gap material at the drain side of the gate is primarily responsible for the current collapse. The negative electron charge accumulated because of this trapping causes a significant depletion of the channel layer, more probably a pinch-off, leading to a drastic reduction of the channel conductance and the decrease of the drain current. This continues to be effective until the drain-source bias is substantially increased, leading to a space-charge injection and giving rise to an increased drain-source current.

With improvements in the material quality available, the transconductance, current capacity, and drain breakdown voltage have all been increased to the point that GaN-based MODFETs are now strong contenders in the arena of high-power devices/amplifiers, particularly at $\mathrm{X}$ band and higher frequencies. As is the case for FET device structure, improved and high resistivity buffer layers have once again played a pivotal role. For chronological purposes, a brief review of the latest class of MODFETs with high transconductances and current levels is given below.

The first breakthrough of N-MODFETs based on GaN came in the 1994-1995 time scale in author's laboratory [71]. These devices with a gate length of $3 \mu \mathrm{m}$ and gate width of $40 \mu \mathrm{m}$, exhibited transconductances of about $120 \mathrm{mS} / \mathrm{mm}$ with low on resistances as they sported doped AlGaN donor layers and low resistance ohmic contacts. The $I-V$ characteristics of a typical device of the kind are shown in Fig. 25. Shortly thereafter, devices with a gate length of $2 \mu \mathrm{m}$, gate width of $40 \mu \mathrm{m}$, and the drain-source separation of $4 \mu \mathrm{m}$ exhibited drain currents of approximately $500 \mathrm{~mA} / \mathrm{mm}$ and extrinsic transconductances of approximately $g_{\mathrm{em}}=185 \mathrm{mS} / \mathrm{mm}$. (a)

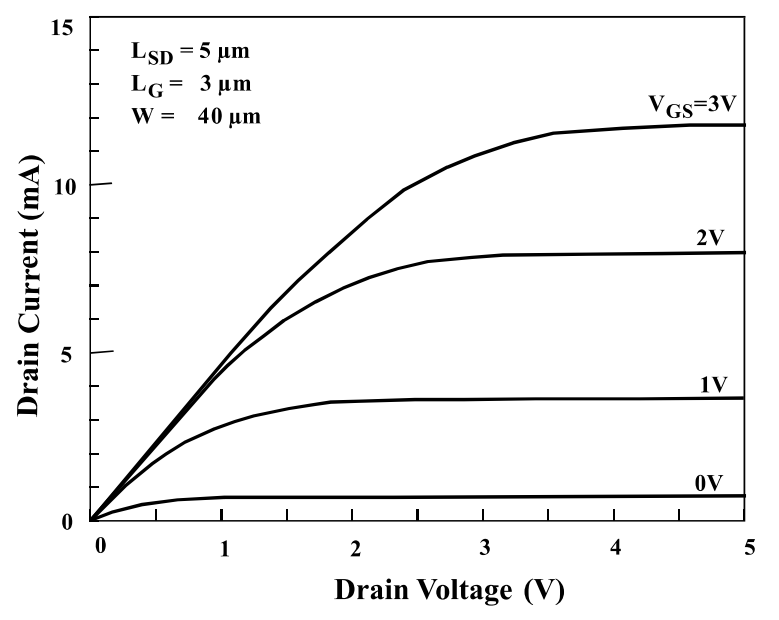

(b)

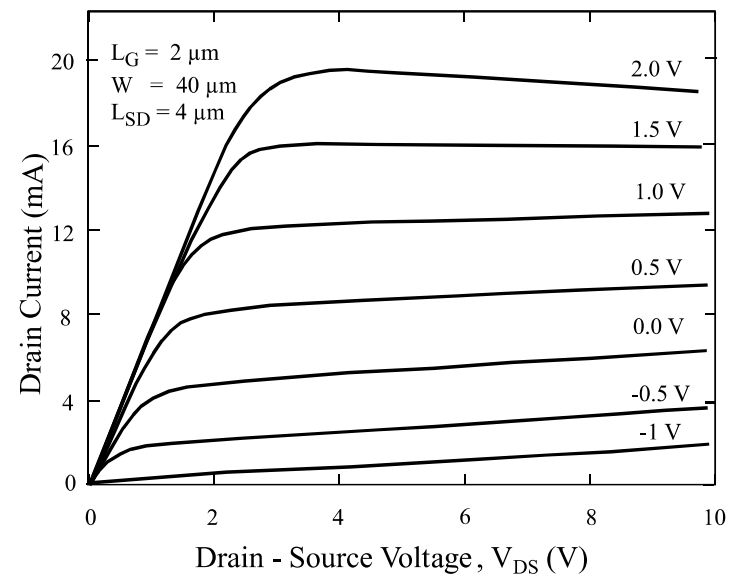

Fig. 25. (a) Output $I-V$ characteristics of the first $\mathrm{GaN}$ MODFET, which exhibited respectable performance. The $3 \mu \mathrm{m}$ gate device had a gate length of $3 \mu \mathrm{m}$ and sported low resistance ohmic contacts and low leakage Schottky barriers. (b) Output $I-V$ characteristics of a GaN MODFET on sapphire with a 2 $\mu \mathrm{m}$ gate, which exhibits negative output conductance due to thermal effects associated with the relatively low thermal conductivity of sapphire.

The drain breakdown voltage for $1 \mu \mathrm{m}$ gate to drain spacing was $\approx 100 \mathrm{~V}$, the exact value depending on the layer design and quality of the layered structure. Soon thereafter, other laboratories achieved similar results in similar structures. What is unique to $\mathrm{AlGaN} / \mathrm{GaN}$ MODFETs as compared to their GaAs varieties is the polarization charge discussed earlier. The polarization issue, particularly the PE variety is used rather liberally. Very high sheet carrier concentrations observed have been ascribed to PE polarization. We have to recognize that ultimately, regardless of the source of the carriers, 
the strength of the electric field that can be accommodated by the semiconductor under the gate without excessive leakage will set an upper limit on the number of carriers at the interface. Use of multi-2DEG structures is one obvious method to increase the current capability of MODFETs, and they have been employed. In those cases, the GaN layer is straddled by two doped AlGaN alloys that donate electrons to the channel, thus increasing the number of electrons available for current conduction. By Hall effect measurement, the mobility and sheet carrier densities in the 2DEG were about 304 $\mathrm{cm}^{2} \mathrm{~V}^{-1} \mathrm{~s}^{-1}$ and $3.7 \times 10^{13} \mathrm{~cm}^{-2}$, respectively, at room temperature. The sheet carrier concentration may have been affected by the PE effect as well. A number of double heterochannel MODFETs (DHCMODFETs) with gate lengths of $1.5-1.75 \mu \mathrm{m}$ and a gate width of 40 $\mu \mathrm{m}$ have been reported.

The maximum drain saturation current $I_{\mathrm{DS}}$ corresponding to a drain-source voltage $V_{\mathrm{DS}}=7 \mathrm{~V}$, and gate source voltage $V_{\mathrm{GS}}=3.5 \mathrm{~V}$ in a DHCMODFET was about $1000 \mathrm{~mA} / \mathrm{mm}$, which is important because in highpower devices, the input is momentarily forward biased. The DHCMODFET had a room temperature extrinsic transconductance of $g_{\mathrm{m}}=270 \mathrm{mS} / \mathrm{mm}$. The value of the total resistance $R_{\mathrm{T}}$ extracted from the linear region of the $I-V$ curves was $4 \Omega / \mathrm{mm}$. Near pinch-off, the drain breakdown voltage was about $80 \mathrm{~V}$, indicating excellent power potential of the device. These measurements were made in a nitrogen-pressurized container to avoid possible oxidation of the contacts and probes. These devices maintain reasonable output characteristics at temperatures as high as $500{ }^{\circ} \mathrm{C}$ with maximum drain current and extrinsic transconductance values of $380 \mathrm{~mA} / \mathrm{mm}$ and 70 $\mathrm{mS} / \mathrm{mm}$, respectively. Cooling to room temperature restored the characteristics, which demonstrates the robustness of this material system and of the metallization employed. It should be noted, however, that high power operation requires large drain breakdown voltages with the added benefit of having large output resistances, which ameliorates impedance matching.

The heat dissipation is a major problem, however, in GaN MODFETs on sapphire substrates as the thermal conductivity of this substrate is about $0.3 \mathrm{~W} \mathrm{~cm}^{-1} \mathrm{~K}^{-1}$, may be even somewhat lower. To make matters worse, the thermal conductivity decreases rapidly as the temperature increases. Consequently, devices show a decreasing drain current (negative differential output conductance) as the drain bias is increased, and needless to say, the power performance is degraded. To overcome this, one must either remove the sapphire substrate followed by mounting the structure on a substrate with better thermal conductivity, employ flip chip mounting, or grow the structure on a substrate with better thermal conductivity. Among the substrates with better thermal conductivity are $\mathrm{Si}$ and in particular $\mathrm{SiC}$. Layers on $\mathrm{Si}$, however, are not of as high quality as one would like, which leaves $\mathrm{SiC}$ substrates, which are expensive and suffer from very inferior surface finish due to the hardness of $\mathrm{SiC}$, as the only good alternative. The early attempts in authors' laboratory to grow $\mathrm{GaN}$ layers on $\mathrm{SiC}$ met with difficulty due to the surface damage roughness, though occasionally very high mobility could be obtained [84].

Two approaches can be employed to remove the surface damage. One is a mechanical chemical polish, which is very slow in coming, and the other is etching in $\mathrm{H}$ and $\mathrm{Cl}$ environment at very high temperatures such as $1500{ }^{\circ} \mathrm{C}$. The authors in collaboration with the group of E. Janzen at Linköping University were able to $\mathrm{H}$ etch Leyl SiC followed by MODFET growth. These devices did not exhibit the negative differential resistance characteristic of sapphire substrates. However, SiC substrates prepared by the sublimation method did not appear to survive this high temperature $\mathrm{H}$ etching process. Researchers have exploited the in situ $\mathrm{H}$ etching process [85] and $\mathrm{HCl}$ etching process [86]. Reports detailing these processes and their effects have appeared in the open literature already. The $I-V$ characteristics of the particular device prepared in 1996 on $\mathrm{SiC}$ substrates are shown in Fig. 26. These results were reported in meetings dealing with the development of high-power devices and the case was made for $\mathrm{SiC}$ substrates as intrinsically being better for GaN power MODFET applications. Several groups participating in those meetings expended a good deal of effort on SiC substrates with initially comparable high-power performance to that on sapphire. Other groups later propelled GaN MODFETs on SiC substrate to their pinnacle with

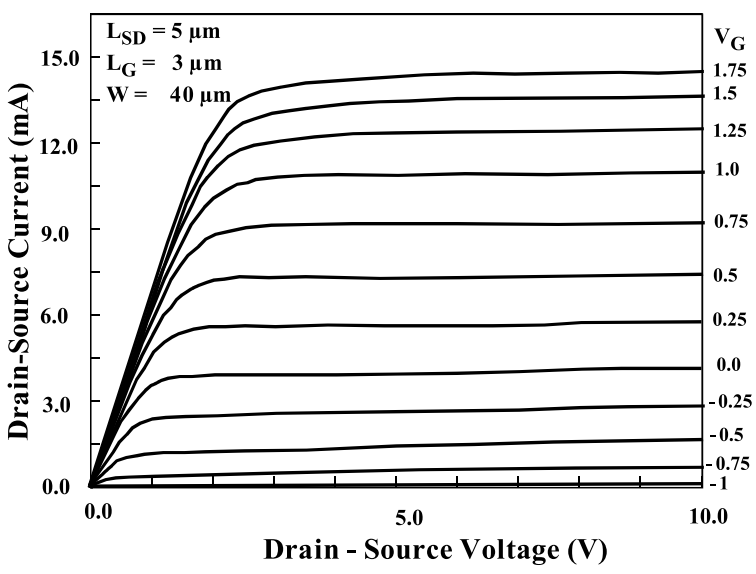

Fig. 26. Output characteristics of a $3 \mu \mathrm{m}$ gate $\mathrm{AlGaN} / \mathrm{GaN}$ MODFET grown on Leyl SiC substrate which is void of the output negative conductance. However, the Leyl substrates are highly conductive and not well suited for FETs due to RF shorting/loading. Nevertheless, experiments of this kind serve to prove the point that the negative output conductance observed in devices on sapphire is most likely due to heat. 
outstanding performance as will be discussed later. It should be pointed out that the pitch of gates for a power FET on a substrate with very good thermal conductivity can be made smaller than on a substrate with inferior thermal conductivity. Consequently, the chip size can be made much smaller in addition to other advantages.

MODFETs have progressed to a point where microwave measurements have been performed on a variety of devices with gate length as wide as $2 \mu \mathrm{m}$ and as narrow as about $0.2 \mu \mathrm{m}$. A typical MODFET structure with $2 \mu \mathrm{m}$ gate lengths has been tested for small-signal Sparameters performed at bias conditions used for the power measurements (i.e., $15 \mathrm{~V},-2.5 \mathrm{~V}$, and $20 \mathrm{~mA}$ for the drain voltage, gate voltage, and drain current, respectively). The unity current gain cutoff frequency $\left(f_{\mathrm{T}}\right)$ and maximum frequency of oscillation $\left(f_{\max }\right)$ were 6 and $11 \mathrm{GHz}$, respectively, at both 15 and $30 \mathrm{~V}$ bias. Values of $f_{\mathrm{T}}$ and $f_{\max }$ in excess of 50 and $100 \mathrm{GHz}$ have been reported for short channel (about $0.2 \mu \mathrm{m}$ ) devices, respectively. As touched upon earlier, devices on sapphire substrates suffer from the low thermal conductivity of sapphire substrates and exhibit negative differential resistance in the output characteristics. Remedies include better heat sinking by flip-chip mounting and the use of high resistivity $4 \mathrm{H}-\mathrm{SiC}$ substrates, which provide good thermal conductivity but are hard to obtain.

There have been a few reports of MODFET power devices on high resistivity $\mathrm{SiC}[25,28,29]$ and p-type $\mathrm{SiC}$ [27] substrates with phenomenal improvement in power handling capability notwithstanding the rapid progress on sapphire substrates. On sapphire, recent $0.7 \mu \mathrm{m}$ gate length $\mathrm{Al}_{0.5} \mathrm{Ga}_{0.5} \mathrm{~N} / \mathrm{GaN}$ MODFETs exhibited a current density of $1 \mathrm{~A} / \mathrm{mm}$, three-terminal breakdown voltages up to $200 \mathrm{~V}$, and $\mathrm{CW}$ power densities of 2.84 and 2.57 $\mathrm{W} / \mathrm{mm}$ at 8 and $10 \mathrm{GHz}$, respectively, representing a marked performance improvement for GaN-based FETs.

Outstanding power levels were achieved with nearhalf-micron gate lengths. With $0.7 \mu \mathrm{m}$ gate length devices on $\mathrm{SiC}$ substrates, where the gate-source spacing and gate-drain spacing were 0.5 and $0.8 \mu \mathrm{m}$, respectively, a total output power of $2.3 \mathrm{~W}$ in a device with a $1.28 \mathrm{~mm}$ gate periphery has been obtained [28]. The power gain at the $2.3 \mathrm{~W}$ output power point was $3.6 \mathrm{~dB}$ with a power added efficiency of $13.3 \%$. The power measurement was conducted at a drain voltage of $33 \mathrm{~V}$. The current and power gain cut-off frequencies were 15 and $42 \mathrm{GHz}$ respectively. The contact resistance, though not the best, was between 2.6 and $3.5 \Omega \mathrm{mm}$. The maximum normalized transconductance was $270 \mathrm{mS} / \mathrm{mm}$ and the drain current was $293 \mathrm{~mA} / \mathrm{mm}$.

Though, excellent results have been reported by many laboratories on $\mathrm{SiC}$ and sapphire substrates, the results that represent the pinnacle are those obtained recently by Nguyen et al. at HRL in devices grown by MBE. The output $I-V$ characteristics of a $0.25 \mu \mathrm{m} \mathrm{Al-}$
GaN MODFET is shown in Fig. 27 and the small signal current and power gains are shown as a function of frequency in Fig. 28. DC characteristics include $600 \mathrm{~mA} /$ $\mathrm{mm}$ current performance and $>60 \mathrm{~V}$ drain breakdown voltage. The current gain cut-off and maximum power gain cut-off frequencies measured were about 40 and 90 $\mathrm{GHz}$, respectively. The minimum noise figure and the associated gain of the same device are shown in Fig. 29 showing a minimum noise figure of $0.85 \mathrm{~dB}$ at $10 \mathrm{GHz}$ with an associated gain of $11 \mathrm{~dB}$, which are simply remarkable. We should mention, however, that even better results are in the offing.

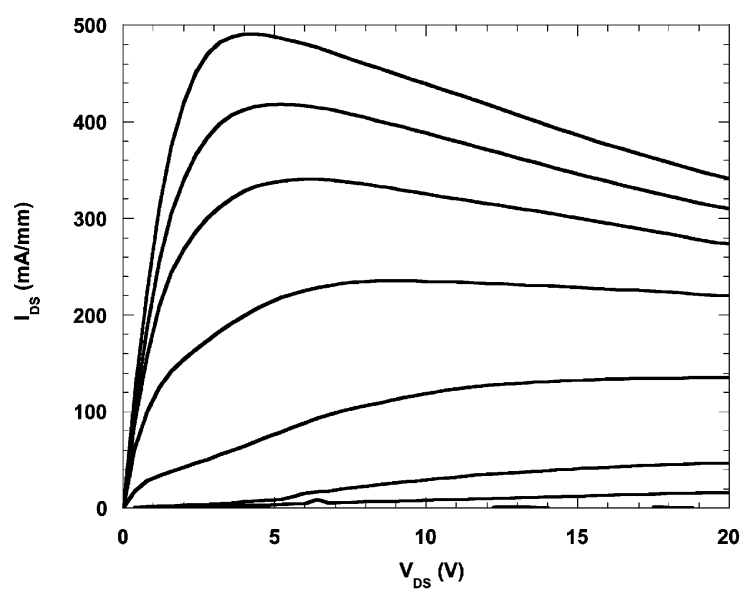

Fig. 27. The output $I-V$ characteristics of a $0.25 \mu \mathrm{m}$ gate AlGaN MODFET on sapphire fabricated at HRL laboratories. Note the negative differential output conductance due to the poor thermal conductivity of sapphire substrate. Courtesy of Drs. N.X. Nguyen and C. Nguyen.

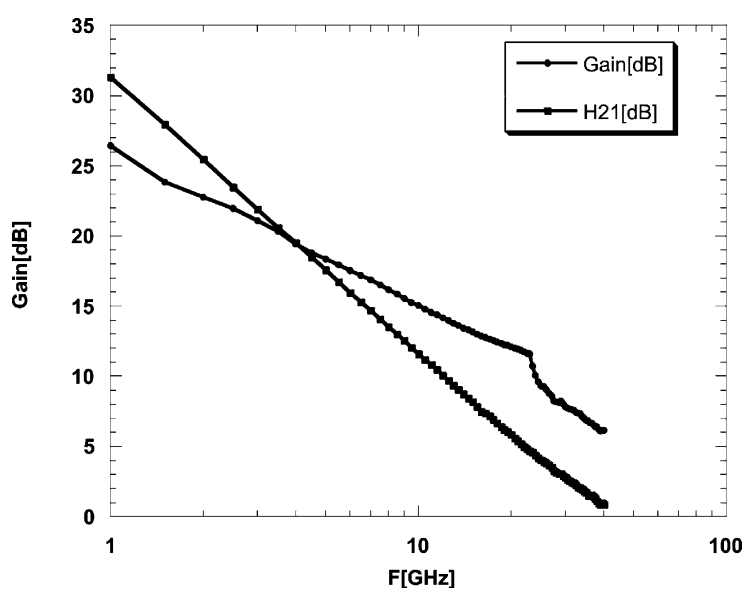

Fig. 28. Small signal current and power gains as a function of frequency of a $0.25 \mu \mathrm{m}$ gate AlGaN MODFET on sapphire fabricated at HRL. Courtesy of Drs. N.X. Nguyen and C. Nguyen. 


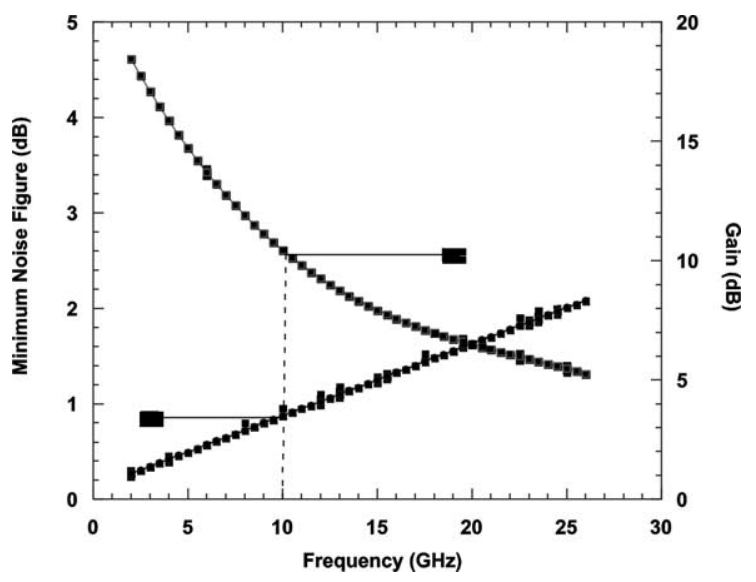

Fig. 29. The minimum noise figure and the associated gain of a $0.25 \mu \mathrm{m}$ gate AlGaN MODFET on sapphire fabricated at HRL. Courtesy of Dr. N.X. Nguyen and C. Nguyen.

The power performance was at $10 \mathrm{GHz}$ from a $1 \mathrm{~mm}$ wide transistor device. This is the highest output power density reported for a $1 \mathrm{~mm}$ or larger power device. More importantly, the power density remained nearly constant as the device size was scaled upward from 0.1 $\mathrm{mm}$ width, where the power exhibited was $6.5 \mathrm{~W} / \mathrm{mm}$, to $1.0 \mathrm{~mm}$. HRL has developed a growth process using molecular beam epitaxy (MBE) which has virtually eliminated material defects common to other reported $\mathrm{GaN}$ devices, thereby enabling the scaling. MBE growth also produces device characteristics with less than $5 \%$ standard deviation over the 2-in. diameter $\mathrm{SiC}$ substrate, a sixfold improvement over previously reported results.

In power devices, the thermal limitation can never be eliminated completely as is the case in nitride devices, particularly when fabricated on sapphire substrates with a thermal conductivity of only $\approx 0.3 \mathrm{~W} \mathrm{~cm}^{-1} \mathrm{~K}^{-1}$. Inclusion of thermal limitations leads to results shown in Fig. 30 for devices that compete in the high-power device arena $[24,68]$. Since new device developments do in general compete with existing and alternative technologies, a brief account of competing technologies for power arena is given below. The Si MESFET analytical curve, modeled for its simplicity, is slightly above the $\mathrm{SiC}$ analytical curve and indicates a maximum power density of $0.35 \mathrm{~W} / \mathrm{mm}$ at $V_{\mathrm{DS}}=7 \mathrm{~V}$ which is slightly lower than $0.39 \mathrm{~W} / \mathrm{mm}$. Since Si RF MESFETs are unavailable, commercial Si RF metal oxide semiconductor FET (MOSFET) results were used for comparison instead. At low voltages, the Si MOSFET data parallel the analytical curve suggesting the validity of the functional dependence of power density on drain voltage. Also shown are two higher power density data points $0.4 \mathrm{~W} / \mathrm{mm}, V_{\mathrm{DS}}=28 \mathrm{~V}$ and $0.87 \mathrm{~W} / \mathrm{mm}, V_{\mathrm{DS}}=48$ $\mathrm{V}$. These higher power densities were obtained with specially designed RF power MOSFETs that incorpo-

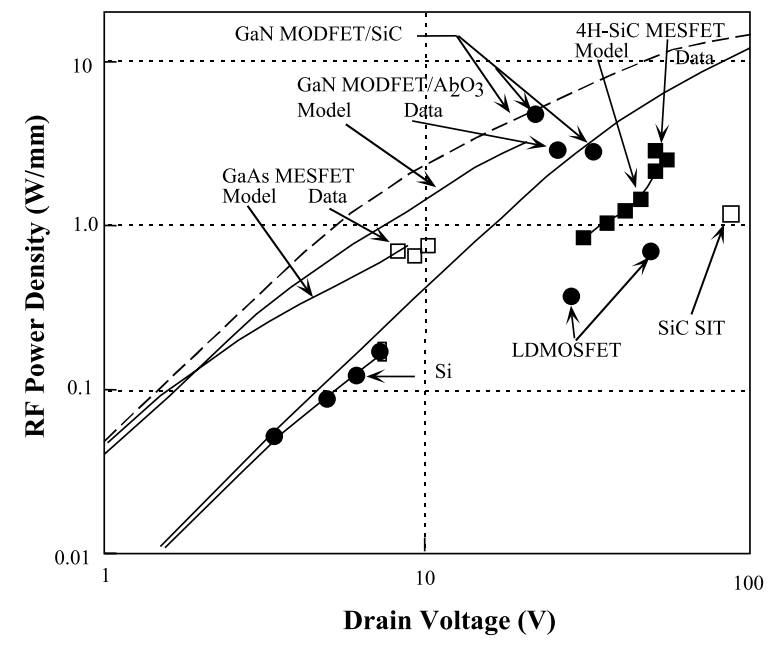

Fig. 30. Simulated and experimental RF power density data for $\mathrm{Si}, \mathrm{GaAs}, \mathrm{SiC}$, and GaN FETs (after Ref. [66]).

rate a lightly doped drain and field plates that significantly increase the breakdown voltage.

The GaAs analytical curve shows the highest power density of all of the devices at the lowest voltages primarily because of the higher electron mobility of GaAs. However, the low breakdown field limits the GaAs MESFET's drain voltage to about $8 \mathrm{~V}$ and power density to $0.63 \mathrm{~W} / \mathrm{mm}$ including thermal effects. Typical commercially available GaAs MESFET power densities are below $1 \mathrm{~W} / \mathrm{mm}$. However, high-performance GaAs FETs with more complex device cross-sections have achieved power densities as high as $1.4 \mathrm{~W} / \mathrm{mm}$ at $18 \mathrm{~V}$. At $100 \mathrm{~V}$, the SiC MESFET has calculated maximum power densities of $7.96 \mathrm{~W} / \mathrm{mm}$ with thermal effects and $9.7 \mathrm{~W} / \mathrm{mm}$ without thermal effects. The highest demonstrated $\mathrm{CW}$ power density, $3.3 \mathrm{~W} / \mathrm{mm}\left(V_{\mathrm{DS}}=50 \mathrm{~V}\right)$ for a $\mathrm{SiC}$ MESFET [87] is also shown for comparison. Additional $\mathrm{SiC}$ data again illustrate the functional dependence of power density on drain voltage. The GaN analytical results are highly dependent on the thermal conductivity of the substrate. With a sapphire substrate, the device is severely thermally limited to $2.24 \mathrm{~W} / \mathrm{mm}$ at $30 \mathrm{~V}$ with a resulting channel temperature of over $400{ }^{\circ} \mathrm{C}$. With a $\mathrm{SiC}$ substrate, however, the analysis predicts that a GaN MODFET could achieve $15.5 \mathrm{~W} / \mathrm{mm}$ at $100 \mathrm{~V}$ with a channel temperature of about $300{ }^{\circ} \mathrm{C}$. We should caution that while the power density figure can be used during the evolution process, eventually the total power figure must prevail. Normalized power density measurements, though frequently reported (a trap the present author also fell into), are often misleading because smaller gate widths naturally lead to larger power densities. This experimental datum point is actually slightly higher than the simulated result, possi- 
bly because of the very small size of the experimental device (100 $\mu \mathrm{m}$ width).

The GaN results of analytical models are highly dependent on the thermal conductivity of the substrate. With a sapphire substrate, the device is severely thermally limited to $2.24 \mathrm{~W} / \mathrm{mm}$ at $30 \mathrm{~V}$ with a resulting channel temperature over $400{ }^{\circ} \mathrm{C}$. However with $\mathrm{SiC}$ substrates [66], the analysis predicts that a $\mathrm{GaN}$ MODFET could achieve $15.5 \mathrm{~W} / \mathrm{mm}$ at $100 \mathrm{~V}$ while keeping the channel temperature at about $300{ }^{\circ} \mathrm{C}$. The key to further improvements lies with our ability to control the polarity of the films, to prepare domain free material, and to reduce defects. If the past few years are any indication, substantial progress is in the wings.

\section{Ultraviolet detectors}

The wavelength range of much interest to a first extent can be divided into IR, visible and UV, each in turn being divided into subsegments. The UV region is divided into a number of sub-UV regions. These regions are near UV (400-300 nm), mid-UV (300-200 nm), far UV (200-100 nm), and extreme UV (100-10 nm) regions. Another scheme classifies these regions as UV-A (400-320 nm), UV-B (320-280 nm), deep UV (350-190 $\mathrm{nm})$, and vacuum UV (200-10 $\mathrm{nm})$ regions.

Efforts to uncover the properties of UV radiation began in the latter half of the 19th century with the realization that the radiation beyond the blue end of the visible spectrum is very important. Spectrographic records obtained at high altitudes underscored the premise that shorter wavelength stellar and solar radiations indeed exist in the UV radiation range. The enthusiasm was, however, thwarted soon thereafter with the observation that the terrestrial atmosphere sets a limit to about $300 \mathrm{~nm}$ on UV light reaching the surface of the earth. UV radiation of $200 \leqslant \lambda \leqslant 300 \mathrm{~nm}$ is absorbed by the ozone layer enveloping the earth, and the UV radiation of $110 \leqslant \lambda \leqslant 250 \mathrm{~nm}$ is absorbed by molecular oxygen, both of which form the terrestrial atmosphere. UV radiation with $\lambda<110 \mathrm{~nm}$ is absorbed essentially by all types of atomic and molecular gases of the atmosphere rendering it non-existent on the surface of the earth. These are the reasons why UV region with $\lambda<200 \mathrm{~nm}$ is generally called the vacuum UV region.

In general, UV detectors may be categorized as photon detectors (photo-detectors) and thermal detectors. The photo-detectors are highly sensitive to radiation and used very widely to measure the rate of photon arrival. Photon detection is through absorption and ensuing creation of free electrons and holes, which lead to a current flow in semiconductors. The change in electrical energy distribution resulting from this absorption of photons gives rise to an observed electrical signal. If they are designed appropriately in a suitable material, they could respond only to UV radiation of certain selected wavelengths. Unlike photo-detectors, the thermal detectors exhibit increased temperature caused by photon absorption. Consequently, these detectors measure the photon flux and yield (for photons of certain selected wavelengths) in the form of a change of certain temperature dependent parameter of the material. Essentially, the radiation can be absorbed even on a black surface coating demonstrating that the thermal effects in a thermal detector are generally wavelength independent. These detectors often find their uses as absolute radiometric standards at UV wavelengths. Among the other types of detectors, the pyroelectric detectors rely on the change of the internal electrical polarization, and the bolometers rely on the change of electrical resistance.

The photo-electric detectors are known to yield a greater stability of response and a greater linearity characteristics. This is achieved by a considerable recent progress in image recording and processing as evidenced by the recently developed photo-voltaic (PV) and photoemissive array detectors. Interestingly, these two detectors can offer, for the first time, the sensitivity and radiometric stability of photo-multipliers, which paves the way to a high-resolution image capability.

\section{Principles of photo-detectors}

To develop insight into the operation of the device, we must follow the trail of photons and created electrons. In a photo-cathode device, the photons are incident on the active region of the material forming the detector. When these photons impact the solid surface of the photo-detector material, electrons are emitted into vacuum providing that the barrier to electron emission is eliminated preferably by a surface treatment, which leads to the generation of current. A voltage is applied between the photo-cathode surface and a positively biased anode to collect the electron and thus measure the ensuing current. Clearly, the magnitude of the photocurrent depends on the quality of the photo-cathode surface. Nevertheless, the photo-electric current flowing through an appropriate circuit is, in general, proportional to the intensity of the incident photon radiation. As the surface material plays a key role in the functioning of a photo-emissive UV detector, the wavelength range of sensitivity is dictated primarily by the absorption band of the cathode material and to some extent the work function of its surface.

In all semiconductor photo-detectors, one or more semiconductor layers are typically grown on a suitable substrate to form a semiconductor photo-detector. The simplest of semiconductor photo-detectors is of the photo-conductive type as shown in Fig. 31. The other type requires a junction, either a $\mathrm{p}-\mathrm{n}$ junction variety or 


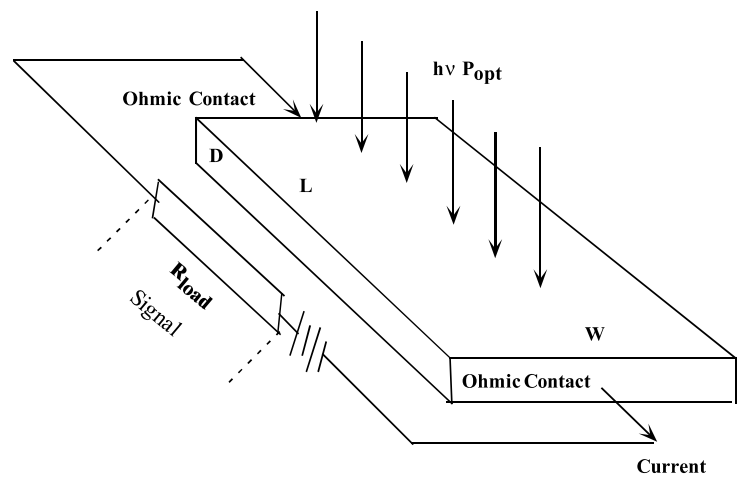

Fig. 31. A schematic representation of a photo-conductive detector with critical dimensions and the external circuit indicated.

a Schottky barrier variety as shown in Fig. 32. In both cases, the light must be allowed to penetrate to the region designated for absorption. This is accomplished by either providing a window in the metal (ohmic contact in the case of the $\mathrm{p}-\mathrm{n}$ junction or Schottky barrier) or the metal is made sufficiently thin to make it semitransparent. The latter is preferred due to series resistance considerations. Heterojunctions can be employed in such a way to reduce the dark current of the device as well as getting the photons of desired wavelengths exactly where the absorptive layer is with minimal photon loss. The Schottky barrier or the $\mathrm{p}-\mathrm{n}$ junction then collects the minority carriers. In the nitride world, the silver lining amongst all the problems induced by the lattice mismatched sapphire substrates is that sapphire is transparent to wavelengths of interest. This lends itself to the back illumination geometry, which can be used very conveniently with a Si read out circuitry for imaging as shown in Fig. 33. Unlike in photo-emissive detectors (photo-cathode), the photons in semiconductor photodetectors are absorbed in the bulk of the semiconductor material. When the energy of the absorbed photon is large enough to raise the electrons to the conduction band from the valence band, they create holes in the valence band. The electric field present, either due to the built-in potential or the applied voltage, separates electrons and holes causing the terminal current to flow which is proportional to the photon flux. In the case of PV detectors, the electric field generated within the depletion region of the device is caused by the differences in the Fermi levels on either side of the junction.

The fundamentals of detectors to be discussed below follow to a great extent reference [88] and relies to some extent on Refs. $[89,90]$. The performance of a photodetector can be described by electron, $J_{\mathrm{e}}$, and hole, $J_{\mathrm{h}}$, current density equations (two continuity equations for

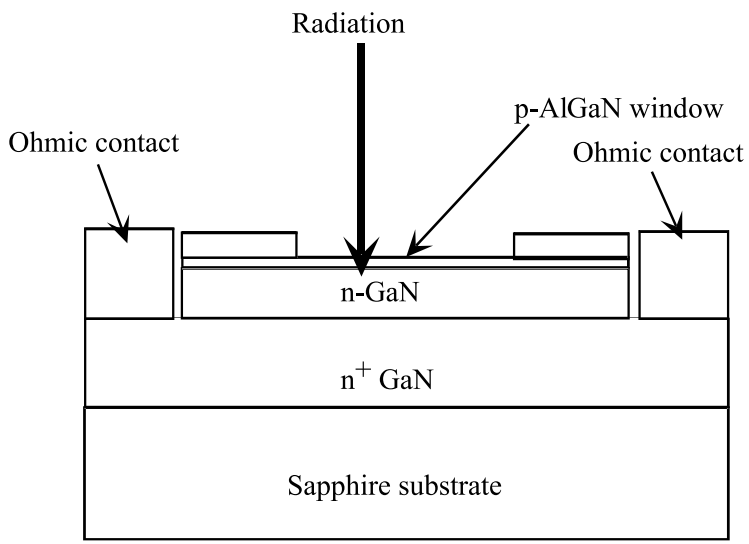

P-n junction detector- Front illimunated

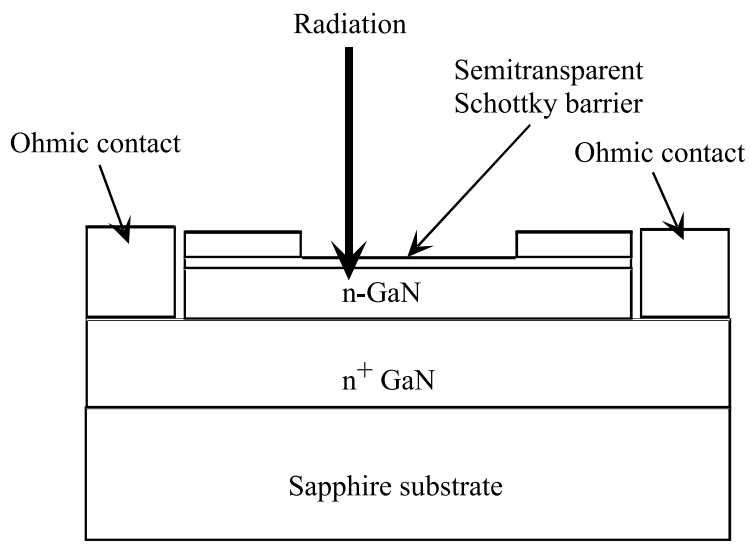

Schottky Barrier detector- Front illimunated

Fig. 32. Schematic representations of a $\mathrm{p}-\mathrm{n}$ junction detector and a Schottky barrier detector.

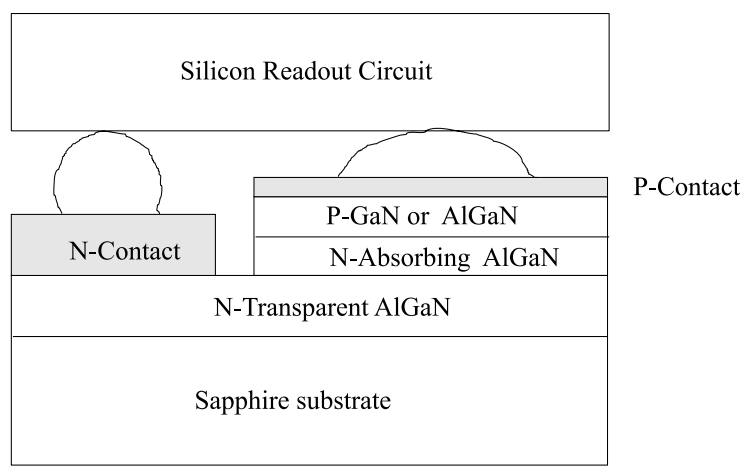

Fig. 33. A schematic of a back illuminated nitride-based detector butted to a $\mathrm{Si}$ read out circuit by In bumping.

electrons and holes, and one Poisson's equation for electrons and holes):

$J_{\mathrm{e}}=q D_{\mathrm{e}}\left(\frac{\mathrm{d} n}{\mathrm{~d} x}\right)-q n \mu_{\mathrm{e}}\left(\frac{\mathrm{d} V}{\mathrm{~d} x}\right)$ 
$J_{\mathrm{h}}=q D_{\mathrm{h}}\left(\frac{\mathrm{d} p}{\mathrm{~d} x}\right)-q n \mu_{\mathrm{h}}\left(\frac{\mathrm{d} V}{\mathrm{~d} x}\right)$

$\frac{1}{q}\left(\frac{\mathrm{d} J_{\mathrm{e}}}{\mathrm{d} x}\right)+(G-R)=0$

$\frac{1}{q}\left(\frac{\mathrm{d} J_{\mathrm{h}}}{\mathrm{d} x}\right)-(G-R)=0$

$\frac{\mathrm{d}^{2} V}{\mathrm{~d} x^{2}}=-\frac{q}{\varepsilon}\left\{N_{\mathrm{d}}-N_{\mathrm{a}}+p-n\right\}$

where $V$ is the electrostatic potential, $N_{\mathrm{d}}$ is the donor concentration, $N_{\mathrm{a}}$ is the acceptor concentrations, $D_{\mathrm{e}}$ and $D_{\mathrm{h}}$ are the electron and hole diffusion coefficients, respectively, $\mathrm{n}$ and $\mathrm{p}$ are the electron and hole concentrations, respectively, and $\varepsilon$ is the dielectric constant of the semiconductor material. The G-R term in Eqs. (16) and (17) accounts for all possible types of generation and recombination components which are very important, particularly in large band gap semiconductors where the minority carrier concentration in the quasineutral regions are negligible. The sources for the $\mathrm{G}-\mathrm{R}$ current are: $\mathrm{SRH}$ recombination, radiative recombination and Auger recombination, at the very least. Lattice defects and impurity energy levels within the energy band gap are the primary causes of SRH recombination. When Eqs. (14)-(18) are solved by a self-consistent iterative procedure, they yield the electrostatic potential $\mathrm{V}$. A reformulation of this $\mathrm{V}$ in terms of integral equations incorporating the necessary boundary conditions and eliminating the current densities $J_{\mathrm{e}}$ and $J_{\mathrm{h}}$ give rise to the carrier densities from the potential distribution.

The quantum efficiency is a very important parameter as it is a measure of how efficiently the photons are converted to current. This parameter, $\eta$, is defined as the number of electron-hole pairs generated per incident photon radiation in a photo-detector. The photo-conductive gain " $g$ " is another parameter of a detector (photo-conductive variety) and is defined as the number of carriers passing through the contact per each generated electron-hole pair. This gain describes how efficiently the generated electron-hole pairs are used to create electric current. The spectral current responsivity of a photo-detector is expressed as the current in amperes divided by the incident power in watts required to generate that current. The expression for the sensitivity can be constructed by recognizing that for each photon, $\eta g$ electrons are generated. Consequently, the responsivity can be expressed as

$R_{i}=\left(\frac{\text { device current }}{\text { optical power }}\right)=\left(\frac{\lambda \eta}{h c}\right) q g$

where $h$ is the Planck's constant, $c$ is the velocity of light, and $q$ is the electronic charge. If the current gain for the photo-current and the noise current is the same, then the current noise resulting from the $\mathrm{G}-\mathrm{R}$ process is

$I_{\mathrm{n}}=q g\left[2 \Omega_{\mathrm{GR}}(\Delta v) A_{\mathrm{e}}\right]^{1 / 2}$

where $\Delta v$ is the bandwidth in terms of frequency, $\Omega_{\mathrm{GR}}$ is the areal thermal $\mathrm{G}-\mathrm{R}, \Omega_{\mathrm{GR}}=\left[t_{\mathrm{e}}(G+R)\right], G$ and $R$ are the generation and recombination rates, respectively, $t_{\mathrm{e}}$ is the thickness of the detector, and $A_{\mathrm{e}}$ is the electron area of the detector. The generation rate $G$ is a combination of the optical generation rate $G_{\mathrm{op}}$ and the thermal generation rate $G_{\mathrm{th}}: G=G_{\mathrm{op}}+G_{\mathrm{th}}$.

If $A_{\mathrm{o}}$ is the optical area of the detector, then the normalized signal-to-noise performance of this detector can be determined by the detectivity, $D^{*}$. The detectivity represents the signal to noise ratio when normalized to a standard size. The $D^{*}$ detectivity is given by

$D^{*}=\frac{R_{i}\left(A_{\mathrm{o}} \Delta v\right)^{1 / 2}}{I_{\mathrm{n}}}$

Eqs. (19)-(21) yield

$D^{*}=\frac{\eta \lambda}{h c}\left\{\frac{A_{\mathrm{o}}}{2 \Omega_{\mathrm{GR}} A_{\mathrm{e}}}\right\}^{1 / 2}$

The photon area and electron area of a photodetector are generally different from having to make contact to the semiconductor which blocks the radiation from reaching the semiconductor. An examination of Eq. (22) indicates that, for a given wavelength $\lambda$, and operating temperature $T$, the highest performance of the photo-detector can be obtained by maximizing $\eta$ / $\left[\Omega_{\mathrm{GR}}\right]^{1 / 2}$. If the recombination process takes place in a region of the device (for example, at the contacts in sweep out photo-conductors (PCs) or in the neutral regions of the diode types) where the photo-electric gain is marginally small, the effect of fluctuating recombination is quite negligible. Because of this, the noise can be low by as much as 2.5 times and the detectivity will be high by the same proportion. The generation process with its associated fluctuation cannot, however, be avoided by any means. The generation and recombination rates are equal at equilibrium. Hence assuming $A_{\mathrm{o}}=A_{\mathrm{e}}$, we get

$D=\frac{\lambda \eta}{2 h c}\left(\frac{1}{G t_{\mathrm{e}}}\right)^{1 / 2}$

In a photo-detector the optical generation $G_{\text {op }}$ may be due to the signal or thermal background radiation. If, for this photo-detector, $G_{\text {th }}$ is at a level where $G_{\text {th }} \ll G_{\text {op }}$, then the NEP may be defined by

$\mathrm{NEP}=\frac{(A \Delta v)^{1 / 2}}{D}$

where $A$ is the area of the device. 


\section{Current and voltage response to incident radiation}

The photo-conductivity in semiconductors under steady state excitation can be described by

$I_{\mathrm{ph}}=q \eta g A \Phi_{\mathrm{s}}$

where $I_{\mathrm{ph}}$ is the short-circuit photo-current at zero frequency (e.g., dc condition), $\Phi_{\mathrm{s}}(\lambda)$ is the photon flux density on the detector with an area of $A=w L$, where $w$ is the width and $L$ is the length of the detector. The term $I_{\mathrm{ph}}$ is the current measured above the dark current limit $I_{\mathrm{dr}}$. As the photo-conductivity is a two-carrier phenomenon, it is composed of the current generated by electrons and holes. Thus,

$I_{\mathrm{ph}}=\frac{w t q\left(\mu_{\mathrm{e}} \Delta n+\mu_{\mathrm{h}} \Delta p\right) V_{\mathrm{a}}}{L}$

where $t$ is the detector thickness which is small as compared to the minority carrier diffusion length, $V_{\mathrm{a}}$ is the applied bias, $\mu_{\mathrm{e}}$ is the electron mobility, $\mu_{\mathrm{h}}$ is the hole mobility, $\Delta n=n-n_{\mathrm{o}}, \Delta p=p-p_{\mathrm{o}}, n$ and $n_{\mathrm{o}}$ are the instantaneous and thermal equilibrium values of the electron concentrations, respectively, and $p$ and $p_{\mathrm{o}}$ are the instantaneous and thermal equilibrium hole concentrations, respectively. Consequently, $\Delta n$ and $\Delta p$ are the excess electron and hole concentrations, respectively.

Nearly in all PCs, the device is designed so that the conductivity is dominated by electrons, and holes play a secondary role. Consequently, if the absorption is uniform and all the photons are absorbed, the rate equation for the excess electron concentration is given by

$\frac{\mathrm{d}}{\mathrm{d} t}(\Delta n)=\frac{\eta \Phi_{\mathrm{s}}}{t}-\frac{\Delta n}{\tau}$

where $\tau$ is the excess carrier lifetime, and under steady state is given by

$\tau=\frac{t \Delta n}{\eta \Phi_{\mathrm{s}}}$

which can be obtained from the steady state generation rate expression of

$\frac{\Delta n}{\tau}=\frac{\eta \Phi_{\mathrm{s}}}{t}$

Eqs. (25) and (26) yield the photo-electric (or photoconductive) current gain $g$ as

$g=\frac{\mu_{\mathrm{e}} t \Delta n V_{\mathrm{a}}}{\eta L^{2} \Phi_{\mathrm{s}}}$

When expressed in terms of the carrier lifetime $\tau$, this photo-electric current gain $g$ is obtained from Eqs. (28a), (28b) and (29) as

$g=\frac{\tau \mu_{\mathrm{e}} V_{\mathrm{a}}}{L^{2}}=\frac{\tau}{L^{2} /\left(\mu_{\mathrm{e}} V_{\mathrm{a}}\right)}=\frac{\tau}{t_{\mathrm{trn}}}$

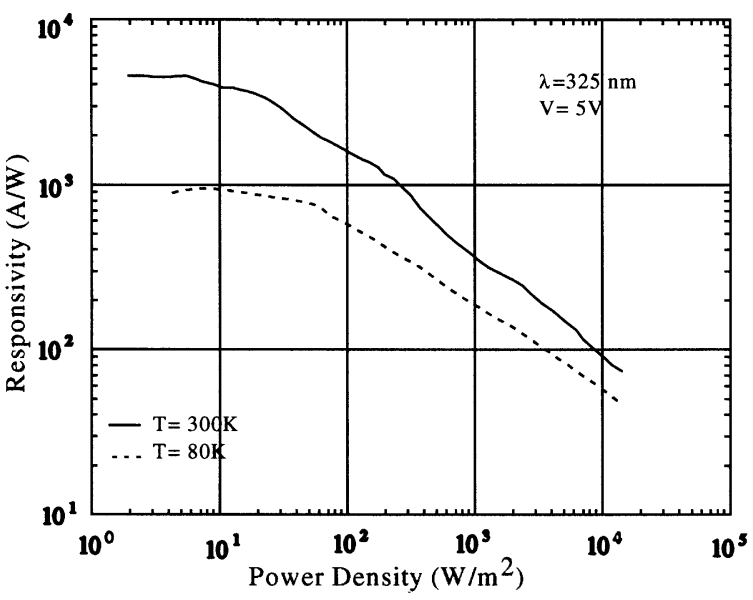

Fig. 34. Responsivity of a GaN PC vs. incident optical power density measured at $325 \mathrm{~nm}$, HeCd laser wavelength, and 300 and $80 \mathrm{~K}$ (after Ref. [100].

where $t_{\text {trn }}$ is the transit time of electrons between ohmic contacts. Eq. (30) suggests that the photo-conductive gain $g$ of a PC is determined by the ratio of the carrier lifetime $\tau$ and the carrier transit time $t_{\text {trn }}$ of the sample electrons. When $\tau>t_{\text {trn }}$, an internal photo-conductive gain results. Since, in general, $\mu_{\mathrm{e}}>\mu_{\mathrm{h}}$, the photo-generated electrons traverse through the semiconductor faster than holes. To preserve charge neutrality, and for current continuity, the external electrode must, therefore supply electrons from the opposite side. These new electrons may move so fast across the detector than the original holes and cannot recombine with them. If this happens, a gain is produced which is proportional to the number of times an electron can transit the detector electrode within its lifetime. This internal photo-conductive gain could be lower than unity if the recombination lifetime is short, and that the electron does not always reach the electrode before recombining with holes. The transit time for a typical $10 \mu \mathrm{m}$ detector is of the order of $10^{-10} \mathrm{~s}$ for an average velocity of about $10^{7}$ $\mathrm{cm} / \mathrm{s}$. Depending on the detector size, material, and doping, the photo-conductive gain can vary from less than one to $10^{5}$. The upper limit is, however, restricted by parameters such as space-charge effects, dielectric breakdown, and ionization effects. Very high value of responsivity at lower power densities (see Fig. 34) obtained for GaN PCs suggests that, for these detectors, the photo-conductive gain was also very high (see Eq. (29)) and that the quantum efficiency is not low either.

\section{Noise in detectors}

Detectors and noise are synonymous in that the minimum detectable radiation power is determined by the noise floor of the detector. The noise sources can be 
categorized into two groups, one being internal to the detector (resulting from one or more sources) and the other being the radiation noise due to the environment (background radiation), both of which are very important. In the limit of the minimum radiant power that is to be detected, all detectors experience a noise wall. The potential sources of noise are fluctuations in the detector itself, in the radiant energy to which the detector responds, or in the electronic system accompanying the detector. Usually, the signal fluctuations and background fluctuations are considered to be responsible for the radiant noise. However, under most operating conditions in the UV, the background fluctuation is minimal, and the radiant noise is dominated by the signal fluctuations. In a photo-detector, the free carriers always exhibit random thermal motion, and because of this, there occurs a fluctuation in the velocity of these carriers, which leads to internal noise. The fluctuations in the density of free carriers due to randomness in the rates of thermal generation and recombination also contribute to the internal noise. There can be four different types of internal noise in a PC. These are Johnson noise, $1 / f$ noise, G-R noise, and preamplifier noise. All of these noise components are expressed in terms of current across the detector terminals. Noise in the detector helps set the detectivity. Another term used to account for noise in the detector is the NEP which is defined as the optical power resulting in a signal to noise ration of one for a bandwidth of one. In other words, it is given by noise divided by the responsivity of the detector.

$\mathrm{NEP}=$ noise $/$ responsivity

We should mention that the maximum responsivity (corresponding to an external quantum efficiency of $100 \%$ ) reduces with increasing photon energy (decreasing photon flux for a given optical power). The maximum responsivity values are 0.2906 and 0.2300 at 360 and $285 \mathrm{~nm}$, respectively, with no gain offered by the detector.

In background limited detectors, meaning when the background radiation noise is dominant, the NEP is given by

$\mathrm{NEP}=h v\left[\frac{2 A \Phi_{\mathrm{B}} \Delta v}{\eta}\right]^{1 / 2}$

where $\Phi_{\mathrm{B}}$ is the total background radiation impinging on the detector (expressed as background radiation flux density (photons $/ \mathrm{s} \mathrm{cm}^{2} \mathrm{~nm}$ )) and the detectivity in a PV detector (operation at zero bias) is given by

$D^{*}=R_{l}\left[\left(4 k T / R_{0} A\right)+2 q^{2} \eta_{\mathrm{ext}} \Phi_{\mathrm{b}}\right]^{-1 / 2}\left(\mathrm{~cm} \mathrm{~Hz}^{1 / 2} \mathrm{~W}^{-1}\right)$

where $R_{0}$ is the zero-bias resistance of the detector. In cases where the total background radiation is very small compared to the noise generated in the detector, the thermal noise limited detectivity is given by

$D^{*}=R_{l}\left(R_{0} A / 4 k T\right)^{1 / 2}\left(\mathrm{~cm} \mathrm{~Hz}^{1 / 2} \mathrm{~W}^{-1}\right)$

In cases where the detector is background limited, which solar blind detector seeks to avoid, the detectivity is given by

$D^{*}=R_{l}\left(2 q^{2} \eta_{\mathrm{ext}} \Phi_{\mathrm{b}}\right)^{-1 / 2}$

\section{Quantum efficiency in detectors}

In a well-designed photo-detector, nearly all photons penetrating the semiconductor are absorbed and they result in carrier generation followed by efficient carrier collection. If all of these processes are done to perfection, the internal quantum efficiency $\eta_{0}$ for this photo-detector is nearly unity. However, under usual circumstances, the actual quantum efficiency which also includes the reflections and other external losses, is lower than the internal quantum efficiency $\eta_{0}$. This is called the external quantum efficiency $\eta_{\text {ext }}$. For example, in a PC there are slabs of material(s) as well as the top and the bottom surfaces all of which exhibit finite reflection coefficients $r_{1}$ and $r_{2}$, respectively. The absorption coefficient $\alpha$ of the slab is also finite. All these lead the internal photo-generated charge profile in the $y$-direction (see Figs. 31 and 35) to be given [91] by $S(y)=S_{1} S_{2}(y)$, where

$S_{1}=\frac{\eta_{0}\left(1-r_{1}\right) \alpha}{1-r_{1} r_{2} \exp (-2 \alpha t)}$

and

$S_{2}(y)=\exp (-\alpha y)+r_{2} \exp [-\alpha(2 t+y)]$

The external quantum efficiency $\eta$ is simply the integral of $S(y)$ over the photo-detector thickness $t$ :

$$
\begin{aligned}
\eta & =\int_{0}^{t} S(y) \mathrm{d} t \\
& =\frac{\eta_{0}\left(1-r_{1}\right)\left[1+r_{2} \exp (-\alpha t)\right][1-\exp (\alpha t)]}{1-r_{1} r_{2} \exp (-2 \alpha t)}
\end{aligned}
$$

If $r_{1}=r_{2}=r$, the external quantum efficiency is reduced to

$\eta_{\text {ext }}=\frac{\eta_{0}(1-r)[1-\exp (\alpha t)]}{1-r \exp (-\alpha t)}$

In a real photo-detector with a well-designed assembly, the reflection capability of only the top surface is very important because the intrinsic detector materials 


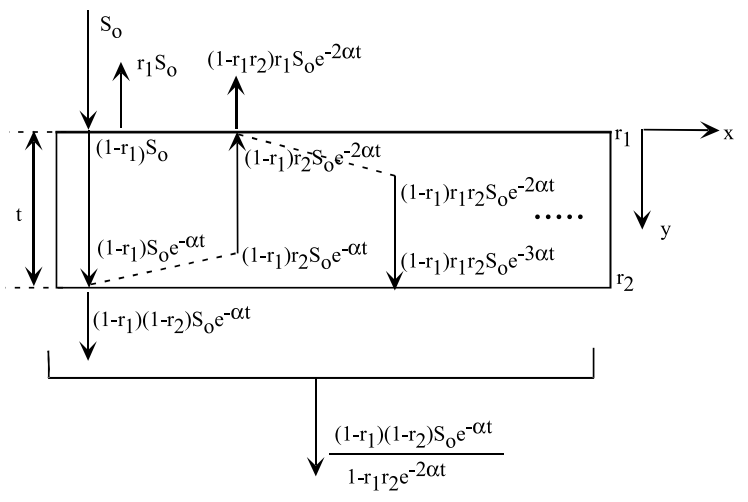

Fig. 35. Surface and internal photo-reflections indicating the intensity of the optical signal within the detector. The optical profile must be multiplied with the absorption coefficient to arrive at the internal charge profile.

tend to be highly absorptive. This allows a simplification of Eq. (37) to

$\eta_{\mathrm{ext}} \approx \eta_{0}(1-r) \approx(1-r)$

\section{Surface and bulk recombination in detectors}

In a typical PC, there is a finite probability of electron-hole recombination at the crystal surface. Owing to this recombination, the total number of steady-state excess carriers is reduced, and the photo-conductive lifetime, $\tau_{\text {eff }}$, then represents the lower limit of the bulk lifetime, $\tau$, of a carrier. The two lifetimes are related by

$\frac{\tau_{\text {eff }}}{\tau}=\frac{\chi}{\alpha^{2} L_{\mathrm{D}}^{2}-1}$

where

$$
\begin{aligned}
\chi & =\alpha L_{\mathrm{D}}\left[\zeta_{\mathrm{A}}+\zeta_{\mathrm{B}} \mathrm{e}^{\alpha t}\left(1-\mathrm{e}^{-\alpha t}\right)\right] \\
\zeta_{\mathrm{A}} & =\frac{\left(\alpha D_{\mathrm{D}}+s_{1}\right)\left\{s_{2} \xi_{\mathrm{A}}+\xi_{\mathrm{B}}\right\}}{\xi_{\mathrm{C}}+\xi_{\mathrm{D}}} \\
\zeta_{\mathrm{B}} & =\frac{\left(\alpha D_{\mathrm{D}}-s_{2}\right)\left\{s_{1} \xi_{\mathrm{A}}+\xi_{\mathrm{B}}\right\}}{\xi_{\mathrm{C}}+\xi_{\mathrm{D}}} \\
\xi_{\mathrm{A}} & =\cosh \left(t / L_{\mathrm{D}}\right)-1 \\
\xi_{\mathrm{B}} & =\left(D_{\mathrm{D}} / L_{\mathrm{D}}\right) \sinh \left(t / L_{\mathrm{D}}\right) \\
\xi_{\mathrm{C}} & =\left(D_{\mathrm{D}} / L_{\mathrm{D}}\right)\left(s_{1}+s_{2}\right) \cosh \left(t / L_{\mathrm{D}}\right) \\
\xi_{\mathrm{D}} & =\left(D_{\mathrm{D}} / L_{\mathrm{D}}\right)^{2}+s_{1} s_{2} \sinh \left(t / L_{\mathrm{D}}\right) \\
D_{\mathrm{D}} & =\frac{D_{\mathrm{e}} p_{0} \mu_{\mathrm{h}}+D_{\mathrm{h}} n_{0} \mu_{\mathrm{e}}}{n_{0} \mu_{\mathrm{e}}+p_{0} \mu_{\mathrm{h}}}
\end{aligned}
$$

Note that $D_{\mathrm{D}}$ is the ambipolar diffusion coefficient, $D_{\mathrm{e}}$ and $D_{\mathrm{h}}$ are the diffusion coefficients of the electrons and holes, respectively, $s_{1}$ and $s_{2}$ are the surface recombination velocities at the front and rear surfaces of the PC, respectively, and $L_{\mathrm{D}}=\left(D_{\mathrm{D}}^{t}\right)^{1 / 2}$. If the absorption coefficient $\alpha$ is large, $\exp (-\alpha t) \approx 0$ and $\mathrm{s}_{1} \ll D_{\mathrm{D}}$. Under this condition, Eq. (39) is simplified to $\frac{\tau_{\text {eff }}}{\tau}=\left(\frac{D_{\mathrm{D}}}{L_{\mathrm{D}}}\right)\left\{\frac{s_{2} \xi_{\mathrm{A}}+\xi_{\mathrm{B}}}{L_{\mathrm{D}} \xi_{\mathrm{C}}+\xi_{\mathrm{D}}}\right\}$

Further simplification based on the assumption that $s_{1}=s_{2}=s$ leads to

$\frac{1}{\tau_{\text {eff }}}=\frac{1}{\tau}+\frac{2 s}{t}$

For some nitrides the predominant recombination mechanism can be indirect, which is mediated by imperfection centers in the crystal. These centers act as recombination centers if carriers reaching such centers have high probability of recombination with carriers of opposite nature, and essentially no probability of returning to a nearby band. In contrast, these centers act essentially as traps if the probability of these carriers to return to the band is high. When these centers act as traps, the total time between the excitation and recombination is essentially identical for electrons and holes. The total time spent out of the traps is, however, different for the electrons and holes, it is only during this time that the carriers contribute to the photo-conductive process. An analysis of decaying characteristics of responsivity as a function of time as well as power density for GaN photo-conductive detectors (see Fig. 36a and b) strongly suggests that it is affected by recombination centers rather than trapping centers. Had it been affected by traps, there would have been re-emission of captured carriers with a strong temperature variation of the emission rate. It is known that, at room temperature, deep recombination centers located several $k T$ from the conduction band exist in GaN grown both by metalorganic chemical vapor deposition (MOCVD) and MBE. Long response time for the photo-response signifies that these centers may indeed be responsible for
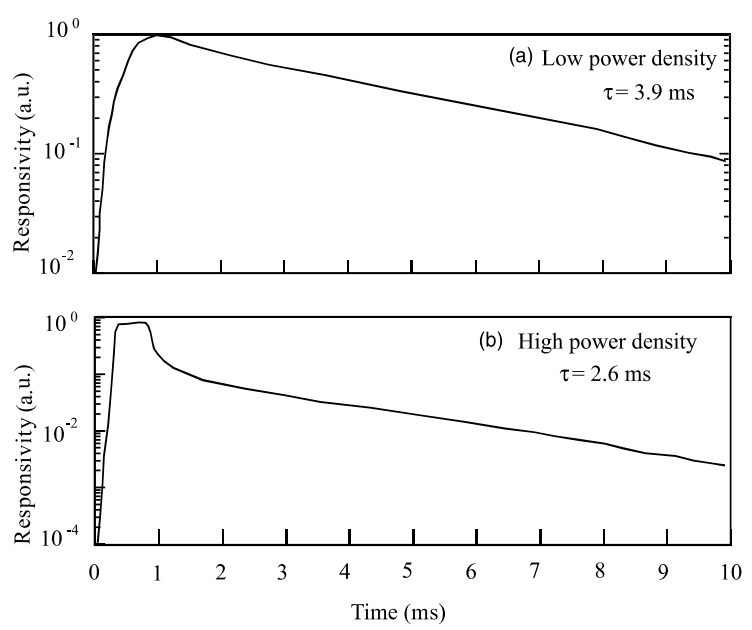

Fig. 36. Evolution of the photo-response of a GaN PC for low (a) and high (b) incident optical power levels (after Ref. [100]). 
controlling photo-response in the said photo-detectors. The response time was very long, because under intense illumination these centers were emptied with ability to cause recombination.

\section{9. $\mathbf{p}-\mathbf{n}$ junction photo-voltaic detectors}

Photo-detectors employing $\mathrm{p}-\mathrm{n}$ junctions with builtin potential barriers at the junction interface are essentially PV in nature if they are used at or near zero bias. The principle of operation is through optically generated excess carrier injection into the vicinity of these barriers. The built-in electric field then drifts the carriers in the appropriate direction, depending on the external circuit. Charge carriers of opposite polarity move in opposite directions. The PV effect can be created in a number of ways. These include $\mathrm{p}-\mathrm{n}$ homojunctions, $\mathrm{p}-\mathrm{n}$ heterojunctions, Schottky barriers, and metal-insulator-semiconductor photo-capacitors. Each of these devices has its own advantages and disadvantages, and the intended application plays a large role in determining which one would be chosen.

Abrupt homojunction is the most common type of a $\mathrm{PV}$ detector. When photons with energy greater than the energy band gap are incident on the front or the back surface of this semiconductor, they create electron-hole pairs in the vicinity of the junction space-charge region. Minority carriers generated within a diffusion length of the junction enter the space-charge region where they are drifted by the strong electric field, as shown in Fig. 37, giving rise to a terminal current as shown in Fig. 38. The equivalent circuit of a $\mathrm{p}-\mathrm{n}$ junction photo-diode is shown in Fig. 39. The total current density of a $p-n$ junction photo-detector may be given by

$J\left(V_{\mathrm{a}}, \Phi\right)=J_{\mathrm{D}}\left(V_{\mathrm{a}}\right)-J_{\mathrm{ph}}(\Phi)$

where $J_{\mathrm{D}}$ is the dark current $J_{\mathrm{ph}}$ is the photo-current, and $V_{\mathrm{a}}$ is the applied bias. Among them, the dark current density $J_{\mathrm{d}}$ depends mainly on the voltage $V_{\mathrm{a}}$, and the photo-current, $J_{\mathrm{ph}}$ depends mainly on the photon flux density $\phi$. The current gain in a PV detector (with the exception of avalanche photo-diodes) is almost equal to 1, and following Eq. (25) the magnitude of the photocurrent is

$I_{\mathrm{ph}}=\eta q A \Phi_{\mathrm{s}}$

Using the above equations, the quantum efficiency of the $\mathrm{p}-\mathrm{n}$ junction photo-diode can be calculated in a straightforward manner.

Under the open-circuit condition of a $\mathrm{p}-\mathrm{n}$ junction photo-diode, the electrons and holes are accumulated on the two sides of the junction producing an open circuit photo-voltage (see Fig. 38). This photo-voltage causes a current flow through the circuit when a load is con-

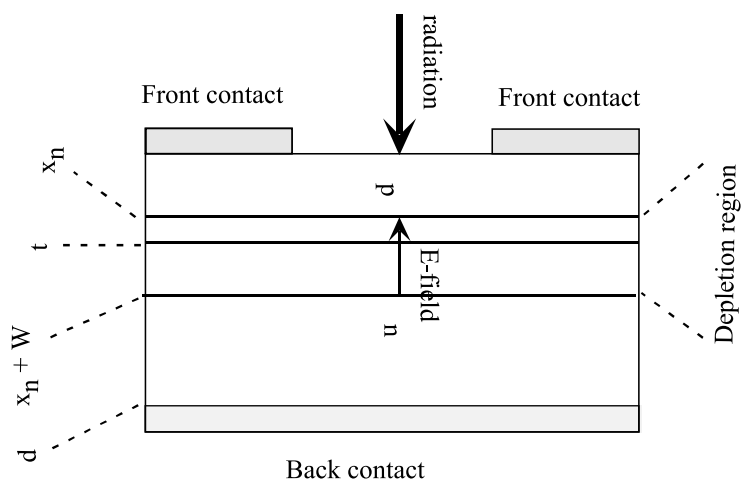

Fig. 37. Schematic representation of a $\mathrm{p}-\mathrm{n}$ junction photo-detector indicating the depletion region and the electric field therein.

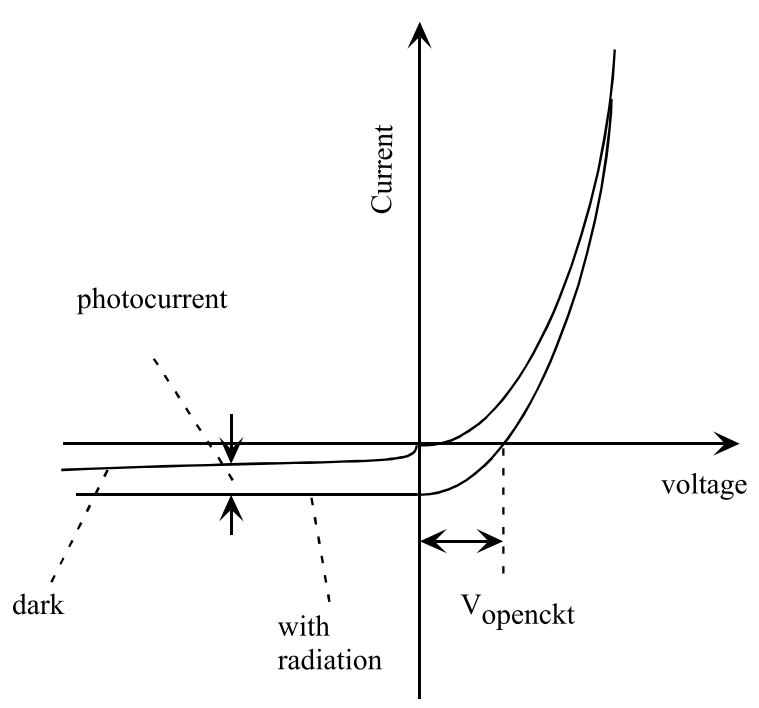

Fig. 38. Terminal current through a $\mathrm{p}-\mathrm{n}$ junction photo-diode in dark and under illumination.

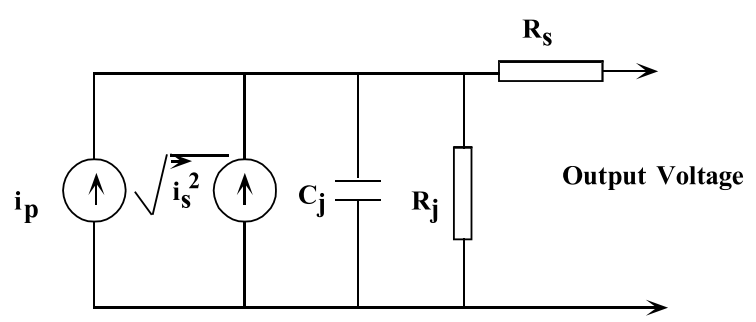

Fig. 39. Equivalent circuit of a p-n junction diode where $I_{\mathrm{p}}$ and $I_{\mathrm{s}}$ represent the photo- and noise-current components, $R_{\mathrm{j}}$ and $R_{\mathrm{s}}$ the junction and series resistances, and $C_{\mathrm{j}}$ the junction capacitance.

nected across the diode. The highest value of this current is obtained if an electrical short is placed across the 
diode terminals. This is called the short-circuit current. The open-circuit voltage is obtained by multiplying the short-circuit current by the incremental (differential) diode resistance $R=(\partial I / \partial V)^{-1}$ at $V=V_{\mathrm{a}}$ :

$V_{\mathrm{ph}}=\eta q A \Phi R$

where $I=I\left(V_{\mathrm{a}}\right)$ is the voltage-dependent current through the photo-detector. For many applications, the photo-detector is operated at zero-bias voltage, yielding

$R_{0}=\left(\frac{\partial I}{\partial V}\right)_{V_{\mathrm{a}}=0}^{-1}$

A frequency-dependent figure of merit for a photodiode is the $R_{0} A$ product as seen in the detectivity expressions, given by

$R_{0} A=\left[\frac{\partial J}{\partial V}\right]_{V_{\mathrm{a}}=0}^{-1}$

where $J=I / A$ is the current density.

The photo-diode can be operated at any point along the $I-V$ characteristic. Reverse bias operation is usually performed for very high frequency applications, which assists in reducing the transit time of the device (through increased carrier velocity) and or allows one to increase the transit region for reducing the diode capacitance, and thus the $\mathrm{RC}$ time constant. The speed is determined by a combination of the transit time and RC time constant and a global optimization approach is often employed.

For $\mathrm{p}-\mathrm{n}$ junction photo-diodes made of wide band gap nitrides, the minority carrier concentrations are very small and thus the diffusion of carriers is not the dominant mechanism for dark current. Several other mechanisms must therefore be involved which are more important. In general though, the dark current is contributed by the bulk of the semiconductor(s), the space charge region and the surface region. The thermally generated current in the bulk and the depletion region arises from (a) diffusion of carriers in the bulk p- and $\mathrm{n}$-regions which is negligible in wide band gap semiconductors, (b) G-R in the depletion region, (c) bandto-band tunneling, (d) trap-to-trap and trap-to-band tunneling, (e) anomalous avalanching of carriers, and (f) ohmic leakage across the space-charge region. On the other hand, surface leakage arises from (a) surface generation current from surface states, (b) generation current in the field-induced surface depletion region, (c) tunneling induced near the surface, (d) ohmic or nonohmic shunt leakage, and (e) avalanche multiplication in a field-induced surface region.

\section{Diffusion current for a $\mathbf{p}-\mathbf{n}$ junction detector}

Diffusion plays a fundamental role in the current conduction mechanism in a $\mathrm{p}-\mathrm{n}$ junction photo-detector. In Fig. 37, which shows a schematic of a p-n abrupt homojunction, the spatial charge of width $w$ surrounds the junction boundary $x=t$. Moreover, there are two quasi-neutral regions within several diffusion lengths of the edges of the depletion regions at $x_{\mathrm{n}}$ and $x_{\mathrm{n}}+w$, which are assumed to be uniformly doped. The dark current is caused by electrons injected from the $n$-side over the potential barrier into the $\mathrm{p}$-side and a similar current due to holes injected from the p-side into the $\mathrm{n}$ side. The diffusion limited minority carrier current is important in small band gap semiconductors, but not to the same extent in large band gap semiconductors as the minority carrier concentration is negligibly small under ideal conditions. The current-voltage characteristics for an ideal diffusion-limited diode is given by

$I_{\mathrm{D}}=A J_{\mathrm{s}}\left[\exp \left(\frac{q V}{k T}\right)-1\right]$

where $J_{\mathrm{s}}$ is the saturation current which, for a thick quasi-neutral region, is given by

$J_{\mathrm{s}}=q\left[\frac{D_{\mathrm{e}} n_{\mathrm{p} 0}}{L_{\mathrm{e}}}+\frac{D_{\mathrm{h}} p_{\mathrm{n} 0}}{L_{\mathrm{h}}}\right]$

When expressed in terms of a one-dimensional device structure depicted in Fig. 37, Eq. (49) may be written, in the case where the layer thicknesses are comparable to the diffusion length, as

$J_{\mathrm{s}}=\frac{q D_{\mathrm{h}} p_{\mathrm{n} 0} U_{\mathrm{h}}}{L_{\mathrm{h}}}+\frac{q D_{\mathrm{h}} n_{\mathrm{p} 0} U_{\mathrm{n}}}{L_{\mathrm{e}}}$

where

$x_{\mathrm{m}}=t+d-x_{\mathrm{n}}-w$

$U_{\mathrm{h}}=\frac{U_{\mathrm{h} 1}}{U_{\mathrm{h} 2}} \equiv \frac{\gamma_{1} \cosh \left(x_{\mathrm{n}} / L_{\mathrm{n}}\right)+\sinh \left(x_{\mathrm{n}} / L_{\mathrm{n}}\right)}{\gamma_{1} \sinh \left(x_{\mathrm{h}} / L_{\mathrm{h}}\right)+\cosh \left(x_{\mathrm{h}} / L_{\mathrm{h}}\right)}$

$U_{\mathrm{e}}=\frac{U_{\mathrm{e} 1}}{U_{\mathrm{e} 2}} \equiv \frac{\gamma_{2} \cosh \left(x_{\mathrm{m}} / L_{\mathrm{e}}\right)+\sinh \left(x_{\mathrm{m}} / L_{\mathrm{e}}\right)}{\gamma_{2} \sinh \left(x_{\mathrm{m}} / L_{\mathrm{e}}\right)+\cosh \left(x_{\mathrm{m}} / L_{\mathrm{e}}\right)}$

Among other parameters, $p_{\mathrm{n} 0}$ is the minority hole concentration on the $\mathrm{n}$-side and $n_{\mathrm{p} 0}$ is the minority electron concentration on the p-side of the photodetector under equilibrium conditions; $s_{1}$ is the surface recombination velocity at the front (illuminated) surface and $s_{2}$ is the surface recombination velocity at the back surface of the photo-detector. $\gamma_{1}=s_{1} L_{\mathrm{h}} / D_{\mathrm{h}}$ and $\gamma_{2}=$ $s_{2} L_{\mathrm{e}} / D_{\mathrm{e}}$. Thus it is apparent from Eqs. (50) and (51a)(51c) that the saturation current density $J_{\mathrm{s}}$ depends on minority carrier diffusion lengths, minority carrier diffusion coefficients, minority carrier concentrations, surface recombination velocities, and junction dimensional 
parameters (e.g., $x_{\mathrm{n}}, t, w$, and $d$ ). For non-degenerate semiconductors, the intrinsic carrier concentration, $n_{\mathrm{i}}=\left(n_{\mathrm{p} 0} p_{\mathrm{n} 0}\right)^{1 / 2}$, diffusivity $D=(k T / q) \mu$, and diffusion length $L=(D \tau)^{1 / 2}$, can be used to reduce Eq. (50) to

$J_{\mathrm{s}}=(k T q)^{1 / 2} n_{\mathrm{i}}^{2}\left[\frac{1}{p_{\mathrm{p} 0}}\left(\frac{\mu_{\mathrm{e}}}{\tau_{\mathrm{e}}}\right)^{1 / 2}+\frac{1}{n_{\mathrm{n} 0}}\left(\frac{\mu_{\mathrm{h}}}{\tau_{\mathrm{h}}}\right)^{1 / 2}\right]^{-1}$

where $p_{\mathrm{p} 0}$ is the majority hole concentration and $\tau_{\mathrm{e}}$ is the electron lifetime on the p-side and $n_{\mathrm{n} 0}$ is the majority electron concentration and $\tau_{\mathrm{h}}$ is the hole lifetime on the n-side of the photo-detector. The temperature dependence of the diffusion current is that for $n_{\mathrm{i}}$.

The resistance at zero bias can be obtained by differentiation of $I-V$ expression of Eq. (48):

$R_{0}=\frac{k T}{q I_{\mathrm{n}}}$

The $R_{0} A$ product, a figure of merit, determined by the diffusion current is obtained as

$$
\left(R_{0} A\right)_{D}=\left(\frac{\mathrm{d} J_{\mathrm{D}}}{\mathrm{d} V}\right)_{V_{\mathrm{a}}=0}^{-1}=\frac{k T}{q J_{\mathrm{s}}}
$$

\section{Quantum efficiency for a $\mathbf{p}-\mathbf{n}$ junction detector}

Three different regions contribute to the quantum efficiency of a photo-detector: two neutral regions, ntype and p-type, and the space-charge region. Thus,

$\eta=\eta_{\mathrm{n}}+\eta_{\mathrm{p}}+\eta_{\mathrm{scr}}$

where

$\eta_{\mathrm{n}}=\frac{(1-r) \alpha L_{\mathrm{h}}}{\alpha^{2} L_{\mathrm{h}}^{2}-1}\left\{\frac{\alpha L_{\mathrm{h}}+\gamma_{1}-U_{\mathrm{h} 1} \mathrm{e}^{-\alpha x_{\mathrm{n}}}}{U_{\mathrm{h} 2}}-\alpha L_{\mathrm{h}} \mathrm{e}^{-\alpha x_{\mathrm{n}}}\right\}$

$\eta_{\mathrm{p}}=\frac{(1-r) \alpha L_{\mathrm{e}}}{\alpha^{2} L_{\mathrm{e}}^{2}-1} \mathrm{e}^{-\alpha\left(x_{\mathrm{n}}+w\right)}\left\{\frac{\left(\gamma_{2}-\alpha L_{\mathrm{e}}\right) \mathrm{e}^{-\alpha x_{\mathrm{m}}}-U_{\mathrm{e} 1}}{U_{\mathrm{e} 2}}\right\}$

$\eta_{\mathrm{scr}}=(1-r)\left\{\mathrm{e}^{-\alpha x_{\mathrm{n}}}-\mathrm{e}^{-\alpha\left(x_{\mathrm{n}}+w\right)}\right\}$

If the loss associated with the optical reflection at the semiconductor surface is neglected, the external quantum efficiency $\eta_{\text {ext }}$ and the internal quantum efficiency $\eta_{0}$ are the same. To obtain a high value for $\eta_{0}$ in a $\mathrm{p}-\mathrm{n}$ junction photo-diode, the illuminated region of the junction must be sufficiently thin so that the carriers generated within this region overcome the junction potential barrier by diffusion and lead to terminal current. Generally, $\mathrm{p}-\mathrm{n}$ junction photo-diodes are designed so as to cause the impinging radiation to be absorbed on one side (e.g., p side) of the junction (see Fig. 37. This is achieved in practice either by making the layer thin or by using a heterojunction in which the energy band gap of the window layer is larger than that of the absorbing layer so that most of the incident radiation can reach the other side of the junction without being absorbed. The meaning of thin needs to be elaborated on. If this layers much thicker than both the absorption depth and the minority carrier diffusion length, while all of the photons would be absorbed, the minority carriers in whole can not diffuse across the junction to lead to a terminal current. On the other hand, if this region is made very thin relative to the absorption depth, assuming that the diffusion length is not the limiting factor here, some of the photon would be absorbed on the other side of the junction. If the diffusion length is the limiting factor, then some of the minority carriers cannot make it across the junction and would not lead to a terminal current. The net effect is a reduction of the internal quantum efficiency. If the back contact is several minority diffusion lengths, $L_{\mathrm{e}}$, away from the junction, then the quantum efficiency is given by

$\eta(\lambda)=(1-r) \frac{\alpha(\lambda) L_{\mathrm{e}}}{1+\alpha(\lambda) L_{\mathrm{e}}}$

If the back contact is less than a diffusion length away from the junction, the back contact has zero surface recombination velocity, and no radiation is reflected from the back surface, then the quantum efficiency would reduce to

$\eta(\lambda)=(1-r)\left\{1-\mathrm{e}^{-\alpha(\lambda) d}\right\}$

where $d$ is the thickness of the p-type region assuming the top layer to be of n-type. If the front-surface reflection is minimized by incorporating an anti-reflection coating, and the device is made thicker than the absorption length (for full absorption of radiation), then the reflection coefficient $r$ would be negligibly small and nearly all of the photons absorbed. Both features allow the quantum efficiency to reach its maximum value.

\section{Noise in a $\mathbf{p}-\mathbf{n}$ junction detector}

The two fundamental processes, viz., fluctuations in the velocity of free carriers due to their random motion and the fluctuations due to randomness of thermal generation and recombination, are less readily identifiable in $\mathrm{p}-\mathrm{n}$ junction photo-diodes than in photo-conductive detectors. The combined effect of these two processes is measurable in the form of shot noise for the minority carriers, which yields the net junction current. This shot noise is the result of the random motion of the minority carriers, which gives rise to fluctuations in the diffusion rates in the neutral regions of the devices and in the $\mathrm{G}-\mathrm{R}$ processes both in the neutral regions as well as the space-charge region. A general theory of noise in $\mathrm{p}-\mathrm{n}$ junction photo-diode developed in terms of all possible 
sources of leakage and arbitrary applied bias is not yet available. An approximate form of the model developed for an ideal diode is

$I_{\mathrm{n}}^{2}=\left[2 q\left(I_{\mathrm{D}}+2 I_{\mathrm{s}}\right)+4 k T\left(G_{\mathrm{j}}-G_{0}\right)\right] \Delta v$

where $I_{\mathrm{D}}=I_{\mathrm{s}}[\exp (q V / k T)-1], v$ is the bandwidth, $G_{\mathrm{j}}$ is the conductance of the junction, and $G_{0}$ is the lowfrequency value of $G_{\mathrm{j}}$. In the low-frequency regime, the second term on the right hand side of Eq. (61) is negligibly small, and thus for a diode in thermal equilibrium (e.g., in absence of applied bias and external photon flux) the mean-square noise current is equal to the Johnson-Nyquist noise:

$I_{\mathrm{n}}^{2}=\frac{4 k T}{R_{0}} \Delta v$

and

$V_{\mathrm{n}}^{2}=4 k T R_{0} \Delta v$

where

$R_{0}^{-1}=q L_{\mathrm{s}} / k T$

The mean-square shot noise in the reverse bias is noted to be half the mean-square Johnson-Nyquist noise at zero bias. However, this predicted improvement in the diode noise in reverse bias is not normally observed in devices due to an increased $1 / f$ noise.

\section{Detectivity for a $\mathbf{p}-\mathbf{n}$ junction detector}

Typically, the photo-electric gain for a $\mathrm{p}-\mathrm{n}$ junction photo-diode is unity, and hence following Eq. (19) the responsivity is given by

$R_{\mathrm{i}}=\frac{q \lambda \eta}{h c}$

Similarly, following Eq. (22) the detectivity or the $D^{*}$, is

$D^{*}=\frac{q \lambda \eta}{h c}\left[\frac{A}{2 q\left(I_{\mathrm{D}}+2 I_{\mathrm{s}}\right)}\right]^{1 / 2}$

Under reverse bias conditions and negligible radiation noise, $I_{\mathrm{D}}$ tends to $-I_{\mathrm{S}}$ and the expression in the bracket of Eq. (65) reduces to $I_{\mathrm{s}}$. This suggests that the performance of an ideal diffusion-limited photo-diode can be optimized by maximizing the quantum efficiency $\eta$ and minimizing the reverse saturation current $I_{\mathrm{s}}$. For a photo-diode, the general expression for the saturation current due to electrons on the $\mathrm{p}$ side is

$I_{\mathrm{s}}^{\mathrm{p}}=\left(\frac{q A D_{\mathrm{e}} n_{\mathrm{p} 0}}{L_{\mathrm{e}}}\right) \frac{\sinh (\theta)+\zeta_{\mathrm{e}} \cosh (\theta)}{\cosh (\theta)+\zeta_{\mathrm{e}} \sinh (\theta)}$

where $\zeta_{\mathrm{e}}=\left(\mathrm{s}_{2} L_{\mathrm{e}} / D_{\mathrm{e}}\right)$ and $\theta=d / L_{\mathrm{e}}$.
The leakage current can be more easily brought to a manageable stage by properly treating the inactive side of the junction, which does not contribute to the postsignal. For example, if the doping or the energy band gap of the inactive side is increased, the minority carrier generation rate and hence the diffusion current will greatly decrease. If the back contact is several diffusion lengths away from the junction, Eq. (66) reduces to

$I_{\mathrm{s}}=\frac{q A D_{\mathrm{e}} n_{\mathrm{p} 0}}{L_{\mathrm{e}}}$

This means that, as the back contact is brought closer to the junction, the leakage current will either increase or decrease, depending on whether the surface recombination velocity is greater than the diffusion velocity $D_{\mathrm{e}} / L_{\mathrm{e}}$. In the limiting case where $d \ll L_{\mathrm{e}}$, the saturation current is reduced by a factor of $d / L_{\mathrm{e}}$ relative to that in Eq. (67) for $s=0$, and increased by a factor of $L_{\mathrm{e}} / d$ for $s=\infty$. If the surface recombination velocity is small, then Eq. (69) is simplified to

$I_{\mathrm{s}}=q G V_{\text {diff }}$

where $G$ is the bulk minority carrier generation rate per unit volume, and $V_{\text {diff }}$ is the effective volume of material from which the minority carriers diffuse into the junction. The effective volume is $A L_{\mathrm{e}}$ for $L_{\mathrm{e}} \ll d$ and tends to $A d$ for $L_{\mathrm{e}} \gg d$. Because of this, the generation-rate for $\mathrm{p}$ type material may be given by

$G_{\mathrm{b}}=\frac{n_{\mathrm{p} 0}}{\tau_{\mathrm{e}}}=\frac{n_{\mathrm{i}}^{2}}{\tau N_{\mathrm{d}}}$

It is, thus, apparent that the device performance of a photo-detector is strongly dependent on the quality of its back contact. In order to alleviate the problems resulting from this back contact, it is advisable to move the back contact many diffusion lengths away to one side, and ensure that the back-side surface is properly passivated. An alternative solution is to design the back contact in such a way that it exhibits a low surface recombination velocity. This can be achieved by introducing a barrier for minority carriers between the metal contact and the rest of the device. An increase in doping concentration or in the energy band gap is a good option for this barrier. Such a barrier, if realized, will effectively isolate the minority carriers from regions where the recombination rates are high such as at the contacts.

\section{Space-charge current in a $\mathbf{p}-\mathbf{n}$ junction detector}

Generally, the space-charge limited current is composed of the G-R current within the space-charge region, the tunneling current in the space-charge region, surface current, and impact ionization current. Each of the components of the space-charge current can be 
characterized by its own current-voltage $(I-V)$ and temperature-voltage $(T-V)$ dependence. Because of this, an accurate solution of the problem is to numerically fit the sum of all the current components to experimental data over a range of both applied voltages and temperatures from which one can determine all the pertinent parameters. Below, we will examine these components of current.

\section{Generation-recombination current}

Even when the width of the space-charge region is much smaller than the minority carrier diffusion length, which is usually the case, the G-R current in this spacecharge region can be more important than the diffusion current, especially at low temperatures and for large band gap semiconductors such as $\mathrm{GaN}$ and AlGaN. This is because the carriers are generated in the spacecharge region due to traps, etc., which cause the generation rate to be much higher than that in the bulk region of the material. Under reverse bias, the $\mathrm{G}-\mathrm{R}$ current may be given by

$I_{\mathrm{GR}}=q G_{\mathrm{scr}} V_{\mathrm{scr}}$

where $G_{\text {scr }}$ is the generation rate and $V_{\text {scr }}$ is the volume of the space-charge region. If the generation rate is dominated by the generation rate from traps in the spacecharge region, then it may be given by $\mathrm{SRH}$ formula as

$G_{\mathrm{scr}}=\frac{n_{\mathrm{i}}^{2}}{n_{1} \tau_{\mathrm{e} 0}+p_{1} \tau_{\mathrm{h} 0}}$

where $n_{1}$ and $p_{1}$ are the electron and hole concentrations, respectively, corresponding to Fermi energy being at the trap energy, and $\tau_{\mathrm{e} 0}$ and $\tau_{\mathrm{h} 0}$ are the carrier lifetimes in the strongly $\mathrm{n}$ - and $\mathrm{p}$-type materials, respectively. If $n_{1} \gg p_{1}$ or vice versa, and the trap level coincides with the intrinsic Fermi level, then

$G_{\mathrm{scr}}=\frac{n_{\mathrm{i}}}{\tau_{0}}$

which leads the G-R current to be

$J_{\mathrm{GR}}=\frac{q w n_{\mathrm{i}}}{\tau_{0}}$

A comparison of Eqs. (72) and (75) indicates that the generation rate $G_{\mathrm{b}}$ in the bulk is a function of $n_{\mathrm{i}}^{2} / N_{\mathrm{d}}$, while the generation rate $G_{\text {scr }}$ for mid-gap states in the space-charge region is a function of $n_{\mathrm{i}}$. Furthermore, unlike the diffusion-limited reverse current, which is independent of the applied bias above a few $k T / q$, the G-R current is weakly dependent on the applied bias. For an abrupt junction where the depletion depth is proportional to $V^{1 / 2}$, it varies as the square root of the applied bias; for a graded junction $\left(w \sim V^{1 / 3}\right)$ it varies as the cube root of the applied bias.

\section{Tunneling current}

Tunneling current is often times the dominant component of the dark current. It can be both direct and indirect. When direct, it arises from the tunneling of electrons across the junction from the valence band to the conduction band without the assistance of the intermediate traps. When indirect, it arises from the tunneling of electrons across the junction from the valence band to the conduction band with the assistance of impurities or defects within the space-charge region. More specifically, the carriers in this process first undergo thermal transitions from one band to the trap states, and then tunnel from the trap state to the other band. Because of this, indirect tunneling requires lower electric fields than the direct tunneling where carriers need to traverse a longer tunneling distance. Assuming a triangular potential under the influence of an applied bias $V_{\mathrm{a}}$, the current $J_{\mathrm{T}}$ resulting from the direct tunneling of electrons of effective mass $m^{*}$ is

$J_{\mathrm{T}}=\frac{q^{2} E V_{\mathrm{a}}}{h^{2}}\left(\frac{2 m^{*}}{E_{\mathrm{g}}}\right)^{1 / 2} \exp \left[-\frac{4\left(2 m^{*} E_{\mathrm{g}}^{3}\right)^{1 / 2}}{3 q E \hbar}\right]$

where $E$ is the electric field, and $h$ is the Planck's constant. For an abrupt $\mathrm{p}-\mathrm{n}$ junction, it is given by

$E=\left[\frac{2 q}{\varepsilon_{\mathrm{s}}}\left(\frac{E_{\mathrm{g}}}{q} \pm V_{\mathrm{a}}\right) \frac{p n}{p+n}\right]^{1 / 2}$

An inspection of Eqs. (74) and (75) suggests that the tunneling current is strongly sensitive to the energy band gap $E_{\mathrm{g}}$, applied bias $V_{\mathrm{a}}$, and the effective doping density $N_{\mathrm{d}}=n p /(n+p)$. It is, however, relatively insensitive to the variation of temperature and the shape of the junction barrier. For a parabolic barrier, the tunneling current $J_{\mathrm{T}}$ is

$J_{\mathrm{T}} \propto \exp \left[-\left(\frac{\pi m^{*} E_{\mathrm{g}}^{3}}{8}\right)^{1 / 2} \frac{1}{q \hbar E}\right]$

\section{Surface leakage current}

Though the surfaces of GaN-based wide band gap semiconductors are more stable than other compound semiconductors, they too exhibit traps and surface states on the surface due to formation of native oxides [92], predominantly $\mathrm{Ga}_{2} \mathrm{O}_{3}$. As revealed by UV and X-ray photo-electron spectroscopy and Auger electron spectroscopy, the surface of, for example, GaN grown on 
sapphire is contaminated with carbon and oxygen. These results must be treated with great precaution to assure that what is measured is not caused by the environment in which experiments are carried out. Assuming care was taken, native oxide such as $\mathrm{Ga}_{2} \mathrm{O}_{3}$ together with some components of a mixed oxynitride of $\mathrm{Ga}$ also forms an overlayer. In fact, it is suspected that due to growth of GaN on sapphire substrate, which is highly lattice mismatched to the epilayer, dangling bonds are present on the GaN surface, and that these dangling bonds are actually responsible for the formation of the $\mathrm{Ga}_{2} \mathrm{O}_{3}$ native oxide layer. What is more, the defects are present in the bulk of the films, which are inevitably a good source of increased dark current. While the exact mechanisms involved in defects participating in the dark current production are still not clear, all of the aforementioned phenomena including Frenkel-Pool current must be considered.

Needless to say, a part of the dark current results from recombination at traps and/or surface states existing on the surface of a GaN-based $\mathrm{p}-\mathrm{n}$ junction, particularly at low temperatures. The SRH recombination mechanism dominates this recombination creating minority carriers. These minority carriers, together with the additional charges existing on the surface and affecting the position of the space-charge region at the surface, participate in current conduction. Both diffusion and drift are responsible for this current conduction. Fast surface states also act as $\mathrm{G}-\mathrm{R}$ centers, yielding leakage current given by

$I_{\mathrm{GRS}}=\frac{q n_{\mathrm{i}} w_{\mathrm{c}} A_{\mathrm{c}}}{\tau_{0}}$

where $w_{\mathrm{c}}$ is the channel width and $A_{\mathrm{c}}$ is the channel area. Surface breakdown mechanism in areas of high electric field also yields leakage current at the surface. Unlike the $\mathrm{G}-\mathrm{R}$ processes occurring at the surface and within surface channels, this mechanism is largely temperature independent.

\section{Performance of nitride-based detectors}

As mentioned previously, the recent serge of interest in nitride semiconductors is due in part to detectors which are unique and cover the wavelength band much below $400 \mathrm{~nm}$. The sun's radiation in the window of 280-300 nm, is absorbed by the ozone layer, leading to a dark background, as shown in Fig. 40. Detectors operative in this so-called solar blind region would not be affected by the solar radiation, rendering the detection of missile plumes an easier task. The alloy AlGaN with about $50 \% \mathrm{AlN}$ absorbs in the aforementioned region of the spectrum. A detector operating in this region sees a dark background that results in reduced radiation noise and allows the detection of minute emission from certain threats. UV (solar blind) detectors are imperative particularly for the military in that they will enable airborne, sea borne, and ground equipment to detect and warn against the ground-to-air, air-to-air, air-to-ground, and ground-to-ground missile threats. The system, at the heart of which is a UV detector, must be able to detect and track extremely weak signals from rapidly moving threats, which necessitates operation in the solar blind region of the spectrum, 260-290 $\mathrm{nm}$. There are also commercial applications such as in boilers and jet engines, but they do not necessarily require operation in the solar blind region of the spectrum.

The continuing proliferation and increasing lethality of surface-to-air and air-to-air missiles pose serious threats to aircraft and terrestrial vehicles. Countering these threats successfully requires early detection of the missile launch. One promising method of detecting incoming missiles is sensing the UV emissions from the rocket plume. Particularly at altitudes lower than 20,000 $\mathrm{ft}$, the solar-blind region is ideal for this purpose because the solar background radiation is almost entirely absorbed by atmospheric ozone. Thus, the $\sim 3000 \mathrm{~K}$ blackbody emission from a missile plume stands out prominently against a dark background, thereby making false alarm rates very low.

Such a missile threat warning system requires twodimensional arrays of highly sensitive photo-detectors to image the solar-blind UV spectral range. The current solution to this problem uses photo-multiplier-type detectors and optical filters to block out all light except that between 260 and $290 \mathrm{~nm}$. The problems with this approach are the limited sensitivity due to low transmission through the filters and low quantum efficiency of the photo-cathodes, the detection of wavelengths outside the solar-blind region caused by filter roll-off at wavelengths longer than $290 \mathrm{~nm}$, and the large size and weight of the photo-multiplier assembly and associated high-voltage power supply. By comparison, solid state

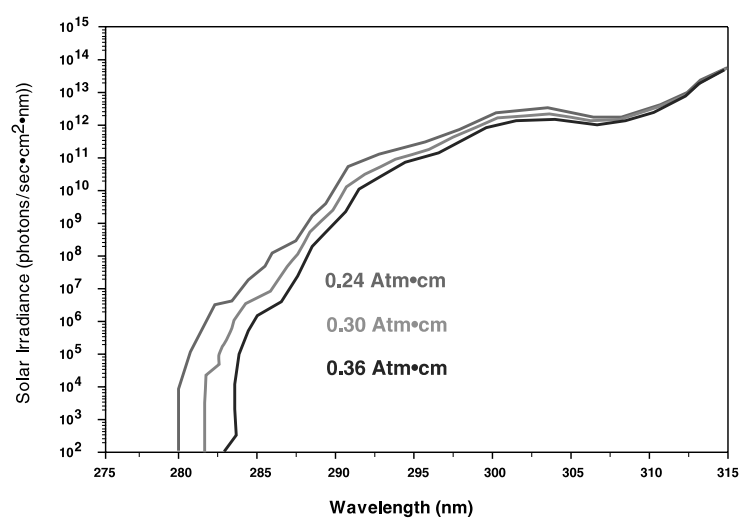

Fig. 40. The spectrum of the Sun's radiation spectrum. 


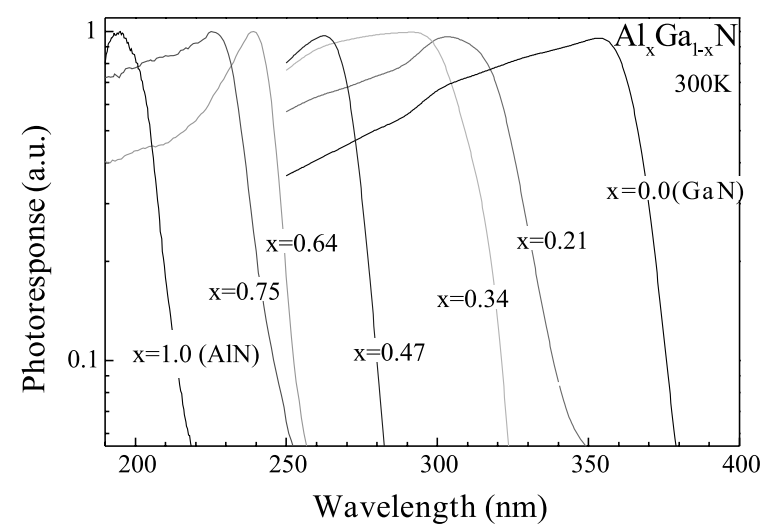

Fig. 41. Room temperature spectral response of AlGaN, including the end binaries, UV photo-conductive detectors showing a tunable cut-off wavelength from 200 to $365 \mathrm{~nm}$. Courtesy of M. Razeghi (after Refs. [92,98]).

detectors offer the advantages of being compact and rugged, having near-unity quantum efficiency, and having better rejection of long wavelength radiation due to their sharp UV band edge. Currently, however, no solid state UV photo-detectors have the sensitivity required for this application.

Numerous papers concerning $\mathrm{GaN}$ and $\mathrm{Al}_{x} \mathrm{Ga}_{1_{-x}} \mathrm{~N}$ UV detectors have appeared in the literature. The detector structures reported include PCs, metal-semiconductor-metals (MSMs), and PVs (including Schottkys, $\mathrm{p}-\mathrm{n}$ junctions, and $\mathrm{p}-\mathrm{i}-\mathrm{ns})$. These detector structures are typically grown on sapphire substrates by variations of MBE and MOCVD methods. Improvements in IIInitride materials growth and detector processing have resulted in progressively higher responsivity $\mathrm{PV}$ and PC UV detectors in the last few years [9,10,93-99]. In the past two years, $\mathrm{Al}_{x} \mathrm{Ga}_{1-x} \mathrm{~N} / \mathrm{GaN}$-based detector response times have decreased dramatically from the ms to the ns regime. To illustrate the range of wavelengths that can be detected by the alloy $\mathrm{AlGaN}$ including the end binary compounds [94,100], we present Fig. 41. As can be seen, to detect wavelengths of $280 \mathrm{~nm}$ or shorter, an AlN mole fraction of $50 \%$ must be employed.

\section{Photo-conductive detectors}

The photo-conductive detectors are essentially radiation-sensitive resistors. They respond to radiation by absorbing a photon of energy $E=h v$, greater than the band gap energy $E_{\mathrm{g}}$ of the active semiconductor region and then yielding an electron-hole pair. Under an externally applied electric field, these electrons and holes are separated from each other and cause a change in the electrical conductivity of the semiconductor, which is measured in an external circuit (see Fig. 31). The signal is detected by a change in the voltage developed across the sample.

In a series of experiments, the photo-conductive behavior of some of III-V nitrides has been investigated in some detail. In one of these experiments, visible-blind UV photo-detectors have been fabricated by using $\mathrm{Al}_{x} \mathrm{Ga}_{1-x} \mathrm{~N}$ with varying AlN mole fraction [101]. The active region of the photo-detector was a $0.8 \mu \mathrm{m}$-thick insulating GaN layer deposited on a $0.1 \mu \mathrm{m}$-thick AlN buffer layer. Sapphire was used as the substrate for the structure, and Au, about $5000 \AA$ thick, was used as the contact metal for forming an inter-digitized contact on the epilayer. Using a standard photo-lithographic procedure and a lift-off technique, the inter-digitated electrode, about $3 \mu \mathrm{m}$ wide, $1 \mathrm{~mm}$ long, and having a $10 \mu \mathrm{m}$ spacing, was formed on the epilayer. The purpose was to ease the measurements of photo-conductive response. The spectral responsivity $R_{\mathrm{i}}$ as a function of wavelength $\lambda$ of the photo-detectors is depicted in Fig. 42. As is apparent from this figure, the peak responsivity remains at about $100 \mathrm{~A} / \mathrm{W}$ until the wavelength reaches $365 \mathrm{~nm}$. The variation of detector responsivity $R_{\mathrm{i}}$ as a function of current $I$ for $254 \mathrm{~nm}$ excitation is essentially linear over five orders of incident power radiation.

In another experiment [102], a $\mathrm{GaN}$ film of about 2 $\mu \mathrm{m}$ thick, grown by MOCVD on a sapphire substrate via an AlN buffer layer, was used to measure photodetective nature of $\mathrm{GaN}$ photo-detectors. The spectral steady state responsivity of the detector was measured under an electric field of $3 \mathrm{kV} / \mathrm{cm}$ across the $\mathrm{GaN}$ region. For energy $E$ higher than the GaN energy band gap $E_{\mathrm{g}}$, the spectral responsivity of the detector was rather flat with a value of about $4 \times 10^{3} \mathrm{~A} / \mathrm{W}$. Band edge response dominated by excitonic effect was purportedly responsible for the high value of the spectral responsivity, which exhibited an abrupt exponential cut-off at energy $E<E_{\mathrm{g}}$. As a result, at $0.5 \mu \mathrm{m}$, the responsivity died down to $4 \times 10^{-3} \mathrm{~A} / \mathrm{W}$, demonstrating an impressive wavelength selectivity of the detector for $E>E_{\mathrm{g}}$ and $E<E_{\mathrm{g}}$.

The variation of the photo-conductive responsivity as a function of incident optical power $P$ at two different temperatures, $T=80$ and $300 \mathrm{~K}$, respectively, is shown in Fig. 34. For this, the excitation was made by a $\mathrm{He}-\mathrm{Cd}$ laser $(0.325 \mu \mathrm{m})$ and the attenuation of the incident power was obtained by using optical densities. It was noted that, at low optical densities, the linear increase in photo-current with optical power leads almost to a constant responsivity having a value of $4.5 \times 10^{3} \mathrm{~A} / \mathrm{W}$. However, in low power regime $\left(P \leqslant 40 \mathrm{~W} / \mathrm{m}^{2}\right)$, the responsivity decreased with increasing power as $P^{1 / 2}$. The variation of photo-responsivity as a function of time both at low and high-power densities is displayed in Fig. 36. It is noted that, in the low power range, the responsivity decays almost exponentially with time. However, in high-power range, the decay is almost 


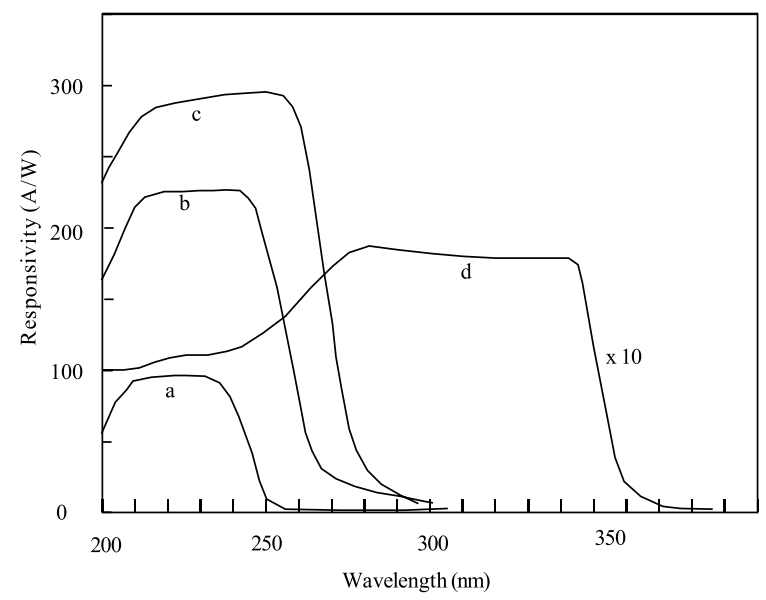

Fig. 42. The spectral responsivity $\mathrm{Ri}$ of a series of $\mathrm{Al}_{x} \mathrm{Ga}_{1-x} \mathrm{~N}$ photo-conductive detectors as a function of wavelength with varying AlN mole fraction, (a) 0.61 , (b) $x=055$, (c) $x=0.46$ and (d) $x=0.05$ (after Ref. [99]).

hyperbolic with less sensitive exponential nature. The variation of the response time with power density at various temperatures (see Fig. 34) is also exponential and that, in the high-power density regime $\left(3 \times 10^{2} \mathrm{~W} /\right.$ $\mathrm{m}^{2}$ ), the response times at various temperatures are essentially identical.

UV detectors of the PV and PC varieties based on nitride semiconductors have been prepared by MBE $[10,103]$ and OMVPE (MOCVD) [9]. As is the case for OMVPE grown materials, UV detectors fabricated in all MBE-grown materials have shown good quantum efficiencies, NEP, and speed of response. We should make a brief mention that standard photo-conducting detectors suffer from large dark current and memory effects. The long delay in turn-off response has been attributed by many to majority carrier traps that are relatively shallow, a small electron capture cross-section coupled with a large hole capture rate $[99,104]$ and unique potential barriers [105]. Munoz et al. [105] argued that the high gain in PC detectors is caused by a modulation mechanism of the conductive volume in the layer. As carriers are photo-generated, they are spatially separated by potential barriers generated by band bending associated with surface and bulk dislocations. Carrier recombination and capture are controlled by such potential barriers and an intrinsic non-exponential recovery process, leading to long delay times and large gains.

Surface-illuminated and back illuminated $\mathrm{GaN}$ and AlGaN/GaN p-i-n UV photo-detectors have been prepared by reactive or gas source MBE and organometallic vapor deposition on sapphire substrates with excellent performance. The p-i-n structures are generally grown on $C$-plane sapphire substrates. Fig. 43 shows the typical structure of a $\mathrm{GaN}$ detector. In the configuration shown, the light is coupled from the top. The thickness of the GaN layer can be adjusted for the light to be absorbed entirely in the top p-type GaN layer or the lightly and unintentionally n-doped GaN layer (referred to as the i-layer) or both. AlGaN (AlGaN homojunction), $\mathrm{GaN}$ (GaN homojunction), and $\mathrm{GaN}$ with $\mathrm{AlGaN}$ window varieties have been explored. Fig. 44 shows the reverse-bias $I-V$ characteristic curves for a $\mathrm{GaN}$ p-n junction detector at several temperatures at and above room temperature. At a reverse bias of $10 \mathrm{~V}$, the leakage current is about mid- $10^{-6} \mathrm{~cm}^{-2}$ which is remarkable for a semiconductor laden with defects.

Fig. 45 shows the spectral response of the GaN UV detector similar to that shown in Fig. 44 but with an $\mathrm{Al}_{0.12} \mathrm{Ga}_{0.88} \mathrm{~N}$ window layer as measured by top illumination at normal incidence for zero bias and $-10 \mathrm{~V}$ bias. At $364 \mathrm{~nm}$, the spectral bandwidth of the monochromator was $\approx 1 \mathrm{~nm}$, and the light intensity on the detectors was approximately $0.06 \mathrm{~mW} / \mathrm{cm}^{2}$. A mechanical chopper modulated the incident light, and a lock-in amplifier recorded the photo-current from the p-i-n photo-diode. A calibrated Si photo-detector was used to measure the spectral intensity of the light source and convert the measured detector current to absolute responsivity. As can be seen, the responsivity drops by more than three orders of magnitude as the incident light's wavelength increases from 360 to $390 \mathrm{~nm}$. Moreover, a reverse bias enhances the response $(0.15 \mathrm{~A} /$ $\mathrm{W}$ at $-10 \mathrm{~V}$ ) while maintaining the sharp cut-off edge and low noise characteristics of the zero-bias responsivity. Fig. 46 shows the spectral response of a recent $\mathrm{p}-\mathrm{n}$ junction $\mathrm{PV}$ detector with a peak responsivity of $0.21 \mathrm{~A} / \mathrm{W}$ at $356 \mathrm{~nm}$ with an internal quantum efficiency of $82 \%$.

An equally important figure of merit for a photodetector is its speed, though certain applications chal-

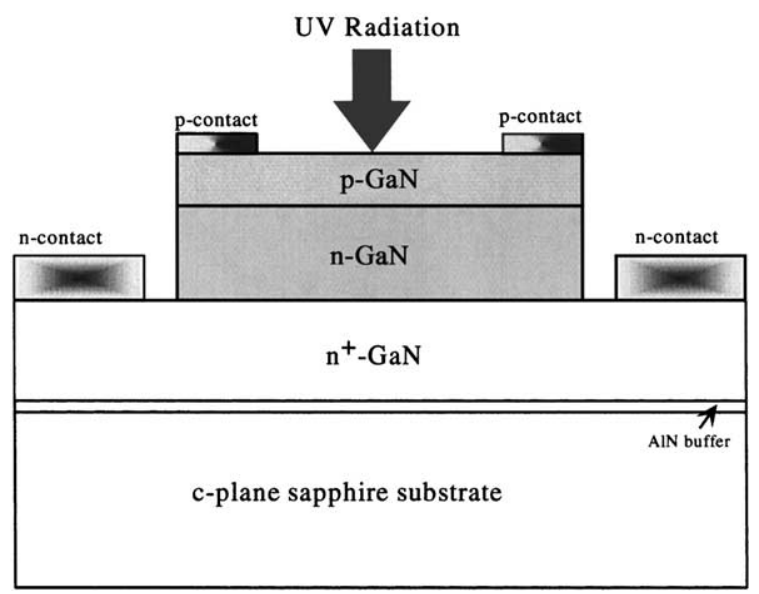

Fig. 43. A drawing of the GaN p-n junction detector prepared by ammonia reactive $\mathrm{MBE}$. 


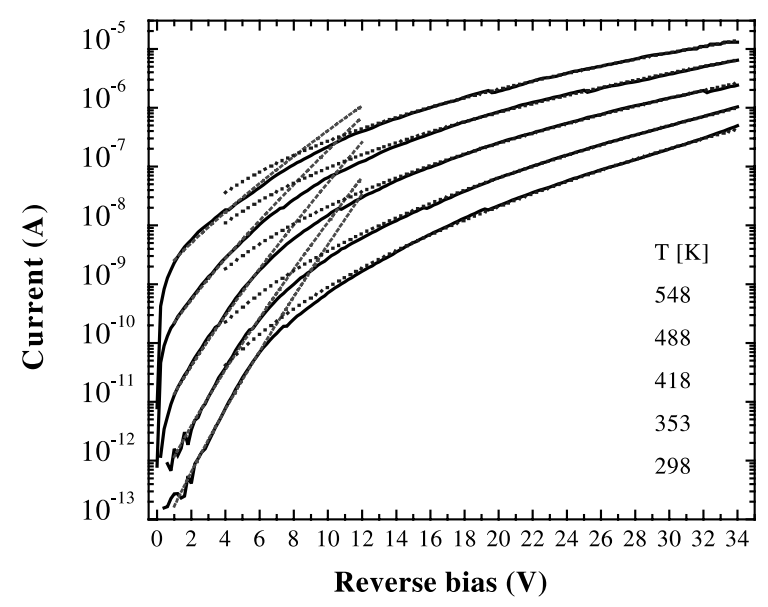

Fig. 44. Current-voltage characteristics of a GaN p-n junction detector at several temperatures. The device was prepared by ammonia reactive MBE. Courtesy of $\mathrm{H}$. Temkin (Texas Tech University).

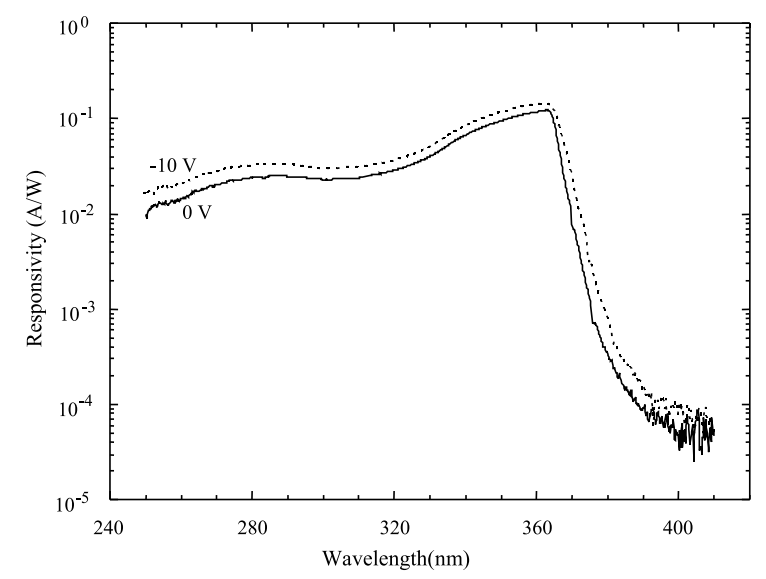

Fig. 45. Spectral response of a GaN UV detector with an $\mathrm{Al}_{0.12} \mathrm{Ga}_{0.88} \mathrm{~N}$ window layer as measured by top illumination at normal incidence for zero bias and $-10 \mathrm{~V}$ bias.

lenge the limits of neither the material system nor the device structure. The time response for the devices incorporating several design features such as all $\mathrm{GaN}$, all $\mathrm{Al}_{x} \mathrm{Ga}_{1-x} \mathrm{~N}$, and heterojunction types at zero bias were measured at $355 \mathrm{~nm}$ using a pulsed-nitrogen laser and by monitoring the decay of the photo-current. The exponential decay times of the photo-current of the GaN, $\mathrm{Al}_{0.05} \mathrm{Ga}_{0.95} \mathrm{~N} / \mathrm{GaN}$, and $\mathrm{Al}_{0.1} \mathrm{Ga}_{0.9} \mathrm{~N}$ p-i-n were found to be about 29, 22, and 12 ns, respectively, as shown in Fig. 47. Fall times as low as 6 ns have been measured [106]. Generally, detectors exhibit two regimes in the photocurrent time decay. At high excitation intensities, trap states in the $\mathrm{GaN}$ band gap presumably become satu-

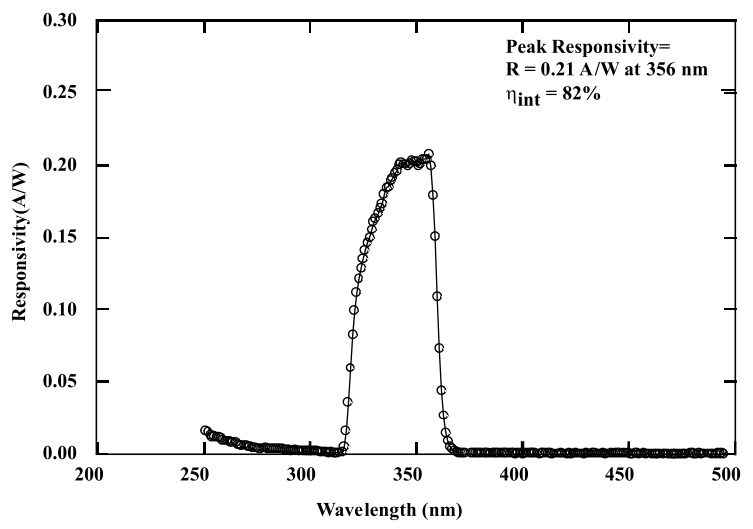

Fig. 46. Spectral responsivity of a PV AlGaN/GaN pin detector with an internal quantum efficiency of $82 \%$ and peak responsivity of $0.21 \mathrm{~A} / \mathrm{W}$. Courtesy of Prof. J. Schetzina (North Carolina State University).

rated and the detectors are then capable of resolving the 8 ns excitation pulse. At lower excitation intensities, a longer time response tail dominates the decay. In this regime, the detectors have a rise time of less than $5 \mathrm{~ns}$ and a fall time $(1 / e)$ of $\approx 31 \mathrm{~ns}$. The recent speed of response data in p-i-n like detectors at a reverse bias voltage of $-10 \mathrm{~V}$ are near or under $1 \mathrm{~ns}$, which are strongly influenced by the series resistance through the p-layer [107]. When the device was illuminated near the center of the window away from the contact area, the decay time increased. The $3-\mathrm{dB}$ point was nearly 1 $\mathrm{GHz}$. A speed of response as low as 90 ps has been reported [108]. We have to reiterate that the speed is determined by convolution of the RC time constant and the transit time. In this regard, $\mathrm{p}-\mathrm{i}-\mathrm{n}$ varieties provide low capacitance due to large depletion region and the transit time can be reduced by the application of a

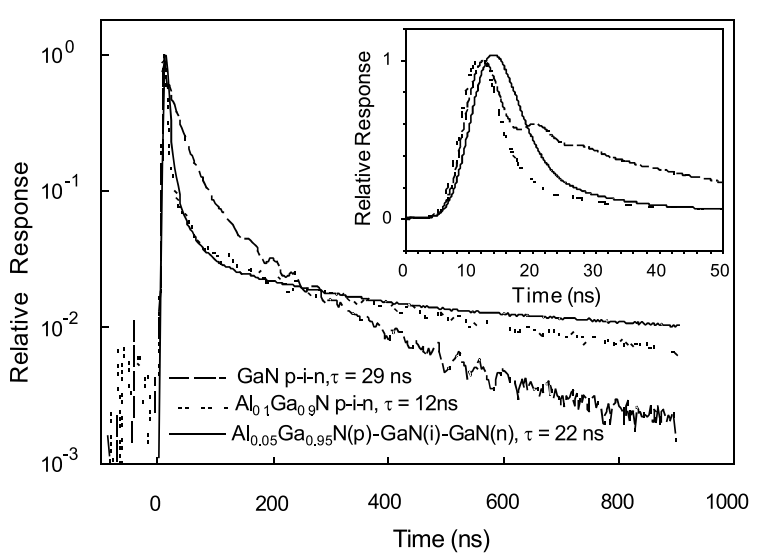

Fig. 47. The time decay of the photo-current for GaN, $\mathrm{Al}_{0.1} \mathrm{Ga}_{0.9} \mathrm{~N} / \mathrm{GaN}, \mathrm{Al}_{0.1} \mathrm{Ga}_{0.9} \mathrm{~N}$ p-i-n detectors. 
reverse bias voltage, which increases the electric field. The caveat is that the dark current would increase with application of a bias voltage if the diode is leaky.

Noise characteristics of the three types of UV detectors ( $\mathrm{GaN}$ homojunction, $\mathrm{Al}_{0.03} \mathrm{Ga}_{0.97} \mathrm{~N}$ homojunction, and $\mathrm{Al}_{0.1} \mathrm{Ga}_{0.9} \mathrm{~N} / \mathrm{GaN}$ heterojunction devices) measured by the group of Prof. H. Temkin at Texas Technical University are tabulated in Table 3. Detector noise for all three devices for small bias values was below the noise floor of the measurement setup. Hence, the noise characteristics were measured at a reverse bias of $28 \mathrm{~V}$. Also tabulated is the corner frequency $f_{\mathrm{c}}$ (i.e., the frequency where the $1 / f$ noise density is equal to the shot noise density). The NEP is then found as usual, by calculating the total rms noise current and dividing it by the responsivity of the device.

Recently, reactive MBE was used by the group of Prof. H. Temkin to produce very low noise GaN p-n junction detectors as shown in Fig. 48. The noise current density measurements were carried out at a reverse bias of $10 \mathrm{~V}$ and at temperatures ranging from the room temperature up to $250{ }^{\circ} \mathrm{C}$. A NEP $(f>100 \mathrm{~Hz})=$ $6.6 \times 10^{-15} \mathrm{~W} / \mathrm{Hz}^{1 / 2}$ was measured at room temperature, which is again remarkable.

While GaN detectors have been used a benchmark, progress in $\mathrm{AlGaN}$ detectors continues at various laboratories. Shown in Fig. 49 is the responsivity of such a detectors with about $300 \mathrm{~nm}$ cut-off wavelength along with the theoretical responsivity curve. The theoretical one assumes perfect quantum efficiency.

For geometrical convenience, speed; and not requiring $\mathrm{p}-\mathrm{n}$ junctions, MSM detectors have been explored in many detector materials and the GaN-based system is no exception. A schematic representation of a typical MSM detector structure with biasing, field lines, depletion front profile and band structure are shown in Fig. 50. Back to back Schottky barriers pave the way for very large dark resistance, [109] unlike the traditional PC detectors. This leads to low dark current [110], which really is a must in high sensitivity detectors with low noise. A dark current of $50 \mathrm{pA}$ at a bias voltage of $10 \mathrm{~V}$ and a responsivity of 0.3 have been reported [109]. The

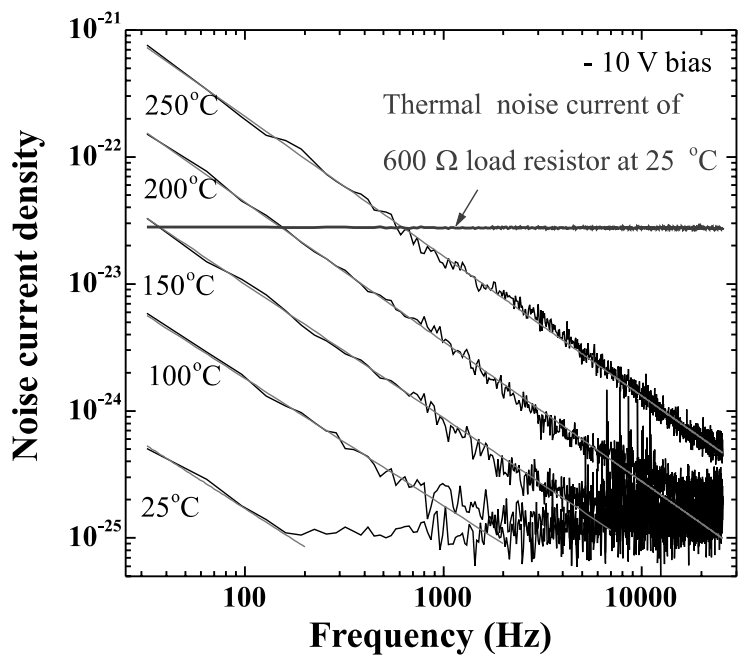

Fig. 48. Thermal noise current density from a GaN p-n junction detector as a function of frequency at temperatures ranging from room temperature to $250{ }^{\circ} \mathrm{C}$. Noise power density for an ammonia reactive $\mathrm{MBE}$ prepared $\mathrm{GaN}$ p-n junction diode. Courtesy of Prof. H. Temkin (Texas Tech University).

rejection ratio is determined by the sharpness of the absorption edge as is the case in $\mathrm{p}-\mathrm{n}$ junction and Schottky barrier vertical detectors. In another report, several $\mathrm{nA}$ of dark current at bias voltages of about $5 \mathrm{~V}$ have been reported along with responsivities of about 0.05 [111]. The MSM detectors are characterized by their high speed [112] as shown in Figs. 51 and 52. Shown in Fig. 51 is the time-domain response of an MSM detector at various bias voltages for $2 \mu \mathrm{m}$ gap spacing. For a bias voltage of $25 \mathrm{~V}$ and without the hole response, $90-10 \%$ decay time is about 200 ps. Shown in Fig. 52 is the frequency-domain response of an MSM detector at $25 \mathrm{~V}$ bias voltages for $2 \mu \mathrm{m}$ gap spacing. For a bias voltage of $25 \mathrm{~V}$, the $3 \mathrm{~dB}$ frequency is $3.8 \mathrm{GHz}$.

Despite the remarkable developments detailed above, true solar-blind detectors with very low noise levels and signal amplification have not yet been reported. The ternary AlGaN with large AlN mole fractions would

Table 3

Noise data for $\mathrm{GaN}$ homojunction, $\mathrm{Al}_{0.03} \mathrm{Ga}_{0.97} \mathrm{~N}$ homojunction, and $\mathrm{Al}_{0.1} \mathrm{Ga}_{0.9} \mathrm{~N} / \mathrm{GaN}$ heterojunction devices

\begin{tabular}{llll}
\hline Parameter & GaN diode & AlGaN diode & AlGaN/GaN diode \\
\hline$I_{\mathrm{d}} @-28 \mathrm{~V}$ & $5 \mathrm{nA}$ & $7 \mathrm{nA}$ & $7 \mathrm{nA}$ \\
$I_{\mathrm{d}} @-2 \mathrm{~V}$ & $7 \mathrm{pA}$ & $7 \mathrm{pA}$ & $7 \mathrm{pA}$ \\
$f_{\mathrm{c}}$ & $158 \mathrm{~Hz}$ & $1683 \mathrm{~Hz}$ & $1683 \mathrm{~Hz}$ \\
NEP (total) & $1.18 \times 10^{-11} \mathrm{~W}$ & $9.1 \times 10^{-12} \mathrm{~W}$ & $2.06 \times 10^{-11} \mathrm{~W}$ \\
NEP @ $10 \mathrm{kHz}$ & $2.14 \times 10^{-14} \mathrm{~W} / \mathrm{Hz}^{1 / 2}$ & $1.25 \times 10^{-14} \mathrm{~W} / \mathrm{Hz}^{1 / 2}$ & $2.49 \times 10^{-14} \mathrm{~W} / \mathrm{Hz}^{1 / 2}$ \\
\hline
\end{tabular}

Notes: Bandwidth was assumed $30 \mathrm{MHz}$ for $\mathrm{GaN}$ and $50 \mathrm{MHz}$ for $\mathrm{AlGaN}$ diodes.

Responsivity was assumed $0.07 \mathrm{~A} / \mathrm{W}$ for $\mathrm{GaN}$ and $0.12 \mathrm{~A} / \mathrm{W}$ for $\mathrm{AlGaN}$ diodes.

$1 / f$ noise power density at $1 \mathrm{~Hz}$ is $8 \times 10^{-18} \mathrm{~A}^{2} / \mathrm{Hz}$ for the $\mathrm{Al}_{0.1} \mathrm{Ga}_{0.9} \mathrm{~N} / \mathrm{GaN}$ diode, $3.8 \times 10^{-21} \mathrm{~A}^{2} / \mathrm{Hz}$ for the $\mathrm{Al}_{0.03} \mathrm{Ga}_{0.97} \mathrm{~N}$ diode, and $1.8 \times 10^{-22} \mathrm{~A}^{2} / \mathrm{Hz}$ for the $\mathrm{GaN}$ diode. 


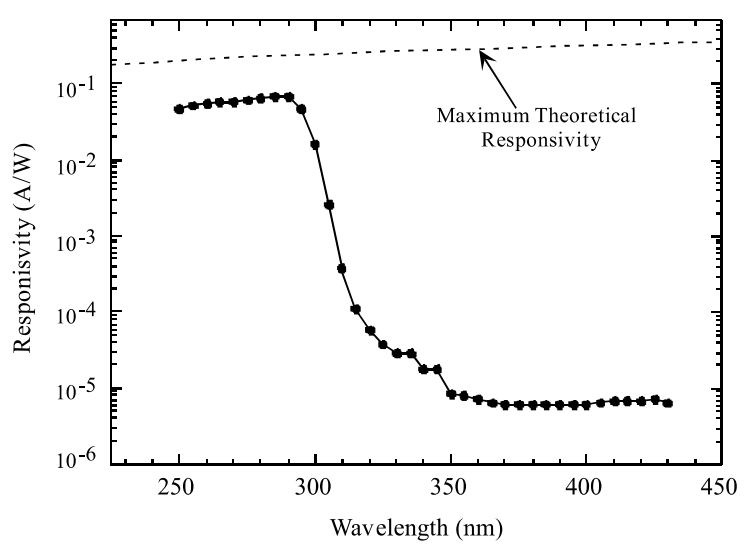

Fig. 49. Responsivity of an AlGaN p-n junction detector with a cut-off wavelength of $300 \mathrm{~nm}$. Courtesy of B. Keller of Nitres.

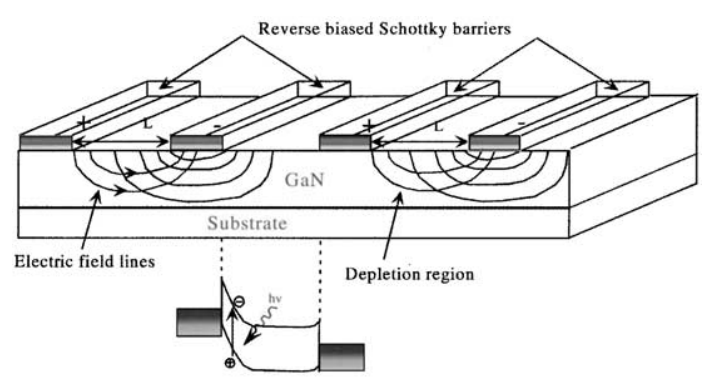

Fig. 50. A schematic representation of a typical MSM detector structure with biasing, field lines, depletion front profile and band structure.

have to be prepared with high quality. If $\mathrm{p}-\mathrm{n}$ junction varieties are to be used, high $\mathrm{p}$ doping in such a high mole fraction $\mathrm{AlGaN}$ is no easy task and none has been demonstrated. The low p doping also requires modifications in the device design in that the large lateral resistance causes the device area to respond non-uniformly. This is in a way similar to the well-known emitter crowding effect in bipolar transistors. Schottky barrier varieties may alleviate the problems associated with $\mathrm{p}$ doping. However, if avalanche photo-diodes are pursued for their much-needed gain, as may be required by some systems applications, the Schottky barrier varieties may not be as successful as the $\mathrm{p}-\mathrm{n}$ junction varieties have been in avalanche photo-diodes based on conventional compound semiconductors. Moreover, very little is really known about the ionization coefficients of electrons and holes in $\mathrm{GaN}$ and its alloys. The calculations are frustrated by the lack of reliable data on the properties (such as complete band structure) of these materials. These difficulties should, however, be viewed as challenges rather than obstacles as has been the case

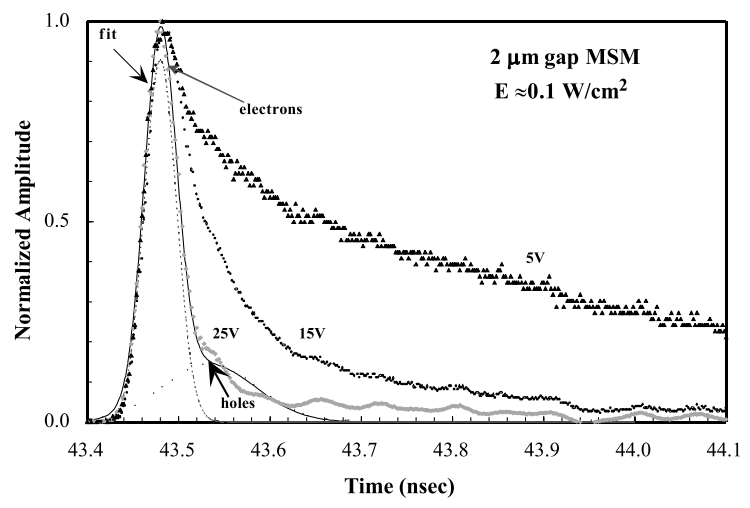

Fig. 51. Time-domain response of an MSM detector at various bias voltages for a $2 \mathrm{~mm}$ gap spacing. For a bias voltage of $25 \mathrm{~V}$ and without the hole response, $90-10 \%$ decay time is about 200 ps. Courtesy of Prof. J.C. Campbell (University of Texas, Austin).

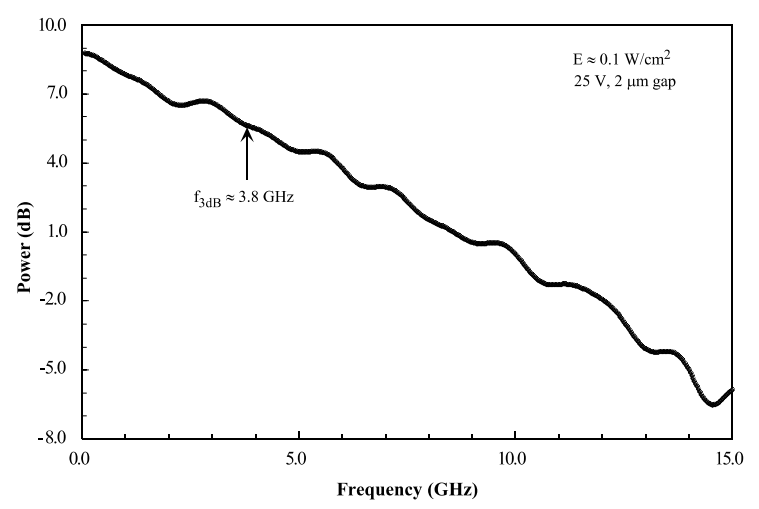

Fig. 52. Frequency-domain response of an MSM detector at 25 $\mathrm{V}$ bias voltages for a $2 \mathrm{~mm}$ gap spacing. For a bias voltage of 25 $\mathrm{V}$, the $3 \mathrm{~dB}$ frequency is $3.8 \mathrm{GHz}$. Courtesy of Prof. J.C. Campbell (University of Texas, Austin).

for just about any advancement. These challenges are being met in the sense that avalanche photo-diodes based on the GaN system have already been reported. A reasonable midway set of performance goals would be to achieve near 80-90\% internal efficiency, $10^{-14} \mathrm{~W}$ NEP, higher than $100 \mathrm{MHz}$ frequency response, 260-290 nm wavelength response, and $10^{-4}$ of peak response relative to response at $300 \mathrm{~nm}$ for $\mathrm{AlGaN}$ detectors.

The single-pixel detector development has begun to give way to arrays for imaging. By combining the spectral signature and image of the source, one can more definitively identify its origin. For example, an $8 \times 8$ GaN Schottky barrier photo-diode array for UV imaging having pixel sizes of $200 \mu \mathrm{m} \times 200 \mu \mathrm{m}$, and responsivity of $0.06 \mathrm{~A} / \mathrm{W}$ has been reported [113]. A purportedly RC time-limited speed of response of $50 \mathrm{~ns}$ was reported. In another effort, a $1 \times 18 \mathrm{GaN}$ MSM 
linear array with a responsivity of $3250 \mathrm{~A} / \mathrm{W}$ at a bias of $10 \mathrm{~V}$ with a response time of $0.5 \pm 0.2 \mathrm{~ms}$ was reported [114]. Most recently, near solar blind large array detectors with Si read-out circuitry already in place has been developed jointly by Prof. Schetzina and his colleagues at North Carolina State University, Honeywell, Inc., and Army Research Laboratories [115].

Specifically, a visible-blind UV camera based on a $32 \times 32$ array of backside-illuminated GaN/AlGaN p-i$\mathrm{n}$ photo-diodes has been demonstrated. Each photo-diode in the array consisted of a base n-type layer of $\mathrm{AlGaN}(\sim 20 \%)$ onto which an undoped $\mathrm{GaN}$ layer followed by a p-type GaN layer was deposited by MOVPE. Due to backside illumination, double-side polished sapphire wafers were used for transparency. The photo-diode array was hybridized to a silicon readout integrated circuit using In bump bonds. This visible-blind digital camera is sensitive to radiation from 320 to $365 \mathrm{~nm}$ in the UV spectral region. Fig. 53 represents a demonstration of a visible-blind UV image from a III-V nitride-based photo-diode array. The image from the FPA (Focal Plane Array) was recorded at NVL on July 28, 1999.

Despite the remarkable developments detailed above, true solar-blind detectors with very low noise levels and signal amplification have not yet been reported. The ternary $\mathrm{AlGaN}$ with large AlN mole fractions would have to be prepared with high quality. If $\mathrm{p}-\mathrm{n}$ junction varieties are to be used, high p-doping in such a high mole fraction $\mathrm{AlGaN}$ is no easy task and none has been demonstrated. The low p-doping also requires modifications in the device design in that the large lateral resistance causes the device area to non-uniformly respond. This is in a way similar to the well-known emitter crowding effect in bipolar transistors. Schottky barrier varieties may alleviate the problems associated with $\mathrm{p}$ doping. However, if avalanche photo-diodes are pursued for their much-needed gain, as may be required by some systems applications, the Schottky barrier varieties may not be as successful as the $\mathrm{p}-\mathrm{n}$ junction varieties have been in avalanche photo-diodes based on conventional compound semiconductors. Moreover, very little is really known about the ionization coefficients of electrons and holes in $\mathrm{GaN}$ and its alloys. The calculations are frustrated by the lack of reliable data on the properties (such as complete band structure) of these materials. These difficulties should, however, be viewed as challenges rather than obstacles as has been the case for just about any advancement. These challenges are being met in the sense that avalanche photo-diodes based on the GaN system have already been reported. A reasonable midway set of performance goals would be to achieve near $80-90 \%$ internal efficiency, $10^{-14} \mathrm{~W}$ NEP, higher than $100 \mathrm{MHz}$ frequency response, 260-290 nm wavelength response, and $10^{-4}$ of peak response relative to response at $300 \mathrm{~nm}$ for $\mathrm{AlGaN}$ detectors.

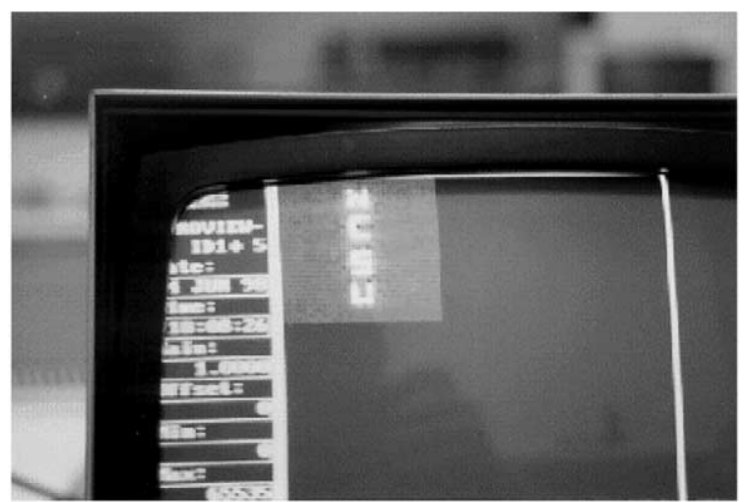

Fig. 53. Demonstration of a visible-blind UV image from a III-V nitride-based photo-diode array. The image from the FPA was recorded at NVL on July 28, 1999. (Courtesy of Prof. J. Schetzina).

\section{Conclusions}

High-power field effect transistors and optical detectors based on the GaN material system have been discussed. The AlGaN/GaN MODFETs with $\mathrm{CW}$ power levels of about $6 \mathrm{~W}$ at $10 \mathrm{GHz}$ for $1 \mathrm{~mm}$ gate periphery have been obtained. A minimum noise figure of $0.85 \mathrm{~dB}$ with an associated gain of $11 \mathrm{~dB}$ at $10 \mathrm{GHz}$ has been obtained. As a precursor to solar-blind detectors that will be operative around $280 \mathrm{~nm}$, where the solar radiation is absorbed by the ozone layer, GaN-based UV detectors with attractive performance have been demonstrated. GaN photo-diodes exhibited zero-bias responsivities of about $0.21 \mathrm{~A} / \mathrm{W}$ at $356 \mathrm{~nm}$, which corresponds to an internal quantum efficiency of $82 \%$. The NEP in photo-detectors prepared by reactive MBE is $(f>100 \mathrm{~Hz}), 6.6 \times 10^{-15} \mathrm{~W} / \mathrm{Hz}^{1 / 2}$, which is extremely small. Finally, the GaN-based detectors with AlN mole fractions appropriate for the solar-blind region of the spectrum have been fabricated into arrays for imaging. Detector arrays with pixel sizes of $32 \times 32$ have been fabricated and tested already. Devices with much better performance are reported almost on a daily basis, which bodes well for the wide band gap nitride material system.

\section{Acknowledgements}

This work was supported by the Air Force Office of Scientific Research Office, the Office of Naval Research, and the National Science foundation and monitored by G.L. Witt (AFOSR) C.E. Wood, Y.S. Park and M. Yoder (ONR), and Verne Hess (NSF). The authors appreciate fruitful discussions with and the assistance of Prof. W. Lambrecht, Dr. M. Estes, Dr. P.J. Schreiber, 
Dr. F. Hamdani, Mr. G. Smith, Dr. C.W. Litton, Dr. D.C. Reynolds, Prof. H. Jiang, Prof. H. Temkin, Prof. J. Schetzina, Prof. A. Salvador, Dr. W. Yang, Dr. B. Keller, Prof. M. Razeghi, Prof. J. Campbell, and Dr. S. Krishnankutty. Prof. B. Gill recommended that this project be undertaken. Prof. F. Bernardini and Dr. O. Ambacher provided many of their preprints. Last but not least, one of us (HM) would like to thank Prof. S.N. Mohammad, Dr. W. Kim, and Mr. F. Stengel for their extensive contributions to the research (and to some extent the manuscript) that led to some of the results reported here.

\section{References}

[1] Morkoç H. Nitride semiconductors and devices. Heidelberg: Springer; 1999.

[2] Mohammad SN, Morkoç H. Progress and prospects of group III-V nitride semiconductors. Prog Quant Electron 1996;20(5 and 6):361-525.

[3] Mohammad SN, Salvador A, Morkoç H. Emerging GaN based devices. Proc IEEE 1995;83:1306-55.

[4] Morkoç H, Strite S, Gao GB, Lin ME, Sverdlov B, Burns M. A review of large bandgap $\mathrm{SiC}$, III-V nitrides, and $\mathrm{ZnSe}$ based II-VI semiconductor structures and devices. J Appl Phys Rev 1994;76(3):1363-98.

[5] Strite ST, Morkoç H. GaN, AlN, and InN: a review. J Vacuum Sci Technol B 1992;10:1237-66.

[6] Ambacher O. Growth and applications of group III nitrides. J Phys D: Appl Phys 1998;31:2653-710.

[7] Pearton SJ, Zolper JC, Shul RJ, Ren F. GaN: processing, defects, and devices. J Appl Phys 1999;86:1-78.

[8] Duffy MT, Wang CC, O'Clock G'D, McFarlane III SH, Zanzucchi PJ. Epitaxial growth and piezoelectric properties of AlN, GaN, and GaAs on sapphire or spinel. J Elect Mat 1973;2:359.

[9] Razeghi M, Rogalski A. Semiconductor ultraviolet detectors. J Appl Phys 1996;79:7433-73.

[10] Xu GY, Salvador A, Kim W, Fan Z, Lu C, Tang H, et al. Ultraviolet photodetectors based on GaN p-i-n and AlGaN (p)-GaN (i)-GaN (n) structures. Appl Phys Lett 1997;71(15):2154-6.

[11] Fritz IJ, Drummond TJ. AIN-GaN Quarter-wave reflector stack grown by gas-source MBE on (100) GaAs. Electron Lett 1995;31:68-9.

[12] Nakamura S, Mukai T, Senoh M. Candela-class high-brightness InGaN/AlGaN double-heterostructure blue light-emitting diodes. Appl Phys Lett 1994;64: 1687-9.

[13] For a review see: Morkoç H, Mohammad SN. High luminosity gallium nitride blue and blue-green light emitting diodes. Sci Magazine 1995;267:51-5.

[14] For a review, see: Morkoç H, Mohammad SN. Light emitting diodes. In: Webster J, editor. Wiley encyclopedia of electrical engineering and electronics engineering. New York: Wiley; 1999.

[15] Nakamura S, Senoh M, Nagahama N, Iwara N, Yamada $\mathrm{T}$, Matsushita $\mathrm{T}$, et al. InGaN/GaN/AlGaN-based laser diodes with modulation-doped strained-layer superlattices. Jpn J Appl Phys 1997;38:L1578.

[16] Morkoç H. Wurtzite GaN based heterostructures by molecular beam epitaxy. IEEE $\mathbf{J}$ selected topics in quantum electronics. Miles R, Akasaki I (editors). 1998;4 (3):537-49.

[17] Schetzina J. Noth Carolina State University, private communication.

[18] Temkin H. Texas Tech University, private communication.

[19] Campbell JC. University of Texas at Austin, private communication.

[20] Schetzina J, et al. Proceedings of the International Conference on Silicon Carbide and Related Materials, 10-15 October, 1999, Research Triangle Park, NC.

[21] Ridley BK. Exact electron momentum-relaxation times in GaN associated with scattering by polar-optical phonons. J Appl Phys 1998;84(7 and 1):4020-1.

[22] Bhapkar UV, Shur MS. Monte Carlo calculation of velocity-field characteristics of wurtize GaN. J Appl Phys 1997;82:1649.

[23] Kolnik J, Oguzman IH, Brennan KF, Wang R, Ruden PP, Wang Y. J Appl Phys 1995;78:1033-8.

[24] Morkoç H. Beyond SiC! III-V nitride based heterostructures and devices. In: Park YS, editor. SiC materials and devices. Willardson and Beer Series, Willardson and Weber, editors. Vol. 52. Academic Press; 1998. p. 307-94 [chapter 8].

[25] Sheppard ST, Doverspike K, Pribble WL, Allen ST, Palmour JW, Kehias LT, et al. High power microwave GaN/AlGaN HEMTs on semi-insulating silicon carbide substrates. IEEE Electron Dev Lett 1999;20(4):161.

[26] Wu Y-F, Keller BP, Fini P, Keller S, Jenkins TJ, Kehias LT, et al. High Al-content AlGaN/GaN MODFET's for ultrahigh performance. IEEE Electron Dev Lett 1998; 19(2):50-3.

[27] Ping AT, Chen Q, Yang JW, Khan MA, Adesida I. DC and microwave performance of high-current $\mathrm{AlGaN} / \mathrm{GaN}$ heterostructure field effect transistors grown on p-type $\mathrm{SiC}$ substrates. IEEE Electron Dev Lett 1998;19(2):54-6.

[28] Sullivan GJ, Chen MY, Higgins JA, Yang JW, Chen Q, Pierson RL, et al. High-power $10-\mathrm{GHz}$ operation of AlGaN HFETs on insulating SiC. IEEE Electron Dev Lett 1998;19:198-9.

[29] Binari S, Redwing JM, Kelner G, Kruppa W. AlGaN/GaN HEMTs grown on SiC substrates. Electron Lett 1997; 33(3):242-3.

[30] Nguyen N, and Nguyen C of HRL Laboratories, private communication.

[31] Henderson T, Aksun MI, Peng CK, Morkoç H, Chao PC, Smith PM, et al. Microwave performance of a quarter micron gate low noise pseudomorphic InGaAs/AlGaAs modulation doped field effect transistor. IEEE Electron Dev Lett 1986;EDL-7:649-51.

[32] Molnar RJ, Goetz W, Romano LT, Johnson NM. Growth of gallium nitride by hydride vapor-phase epitaxy. J Cryst Growth 1997;178(1-2):147-56.

[33] Yamaguchi S, Kariya M, Nitta S, Takeuchi T, Wetzel C, Amano H, et al. Structural properties of InN on GaN grown by metalorganic vapor-phase epitaxy. J Appl Phys 1999;85(11and 1):7682-8.

[34] Morkoç H, Ünlü H, Ji G. Fundamentals and technology of MODFETs. Vols. I and II. Chichesters: Wiley; 1991. 
[35] Bykhovski AD, Kaminski VV, Shur MS, Chen QC, Khan MA. Piezoresistive effect in Wurtzite n-type GaN. Appl Phys Lett 1996;68:818-9.

[36] Bernardini F, Fiorentini V, Vanderbilt D. Spontaneous polarization and piezoelectric constants in III-V nitrides. Phys Rev B 1997;56:R10024.

[37] For a review, see Resta R. Macroscopic polarization in crystalline dielectrics: the geometric phase approach. Rev Mod Phys 1994;66:899, and references therein.

[38] Ambacher O, Smart J, Shealy JR, Weimann NG, Chu K, Murphy M, et al. J Appl Phys 1999;85:3222.

[39] Ambacher O, Foutz B, Smart J, Shealy JR, Weimann NG, Chu K, Murphy M, Sierakowski AJ, Schaff WJ, Eastman LF, Dimitrov R, Mitchell A, Stutzmann M, J Appl Phys 2000;87:334-44.

[40] Smotchkova IP, Elsass CR, Ibbetson JP, Vetury R, Heying B, Fini P, et al. J Appl Phys 1999;86:4520-6.

[41] Daudin B, Rouviere JL, Arley M. Polarity determination of $\mathrm{GaN}$ films by ion channeling and convergent beam electron diffraction. Appl Phys Lett 1996;69:2480.

[42] Vermaut P, Ruterana P, Nouet G, Salvador A, Morkoç H. Structural defects due to interface steps and polytypism in III-V semiconducting materials: a case study using high resolution electron microscopy of the H-AlN/6H-SiC interface. Philos Mag A 1997;75:239-59.

[43] Potin V, Ruterana P, Nouet G, Salvador A, Morkoç H. The atomic structure of the 10-10 inversion domains in GaN/Sapphire layers. In: Abernathy CR, Amano H, Zolper JC, editors. Proc. of Gallium Nitride and Related Materials II Symposium, vol. 468. Pittsburgh, PA: Mater. Res. Soc; 1997. p. 323-8.

[44] Martin GA, Strite S, Botchkarev A, Agarwal A, Rockett A, Morkoç H, et al. Appl Phys Lett 1994;65:610.

[45] Martin GA. Ph.D. Thesis, Semiconductor electronic band alignment at heterojunctions of wurtzite $\mathrm{AlN}, \mathrm{GaN}$ and InN, Department of Physics, University of Illinois, 1996.

[46] Martin GA, Botchkarev A, Agarwal A, Rockett A, Morkoç H. Valence-band discontinuities of Wurtzite $\mathrm{GaN}, \mathrm{AlN}$, and InN heterojunctions measured by X-ray photoemission spectroscopy. Appl Phys Lett 1996;68:2541.

[47] Bernardini F, Fiorentini V. Macroscopic polarization and band offsets at the nitride heterojunctions. Phys Rev B 1998;57(16):1-4.

[48] Bastard G. Wave mechanics applied to semiconductor heterostructures. Edition de Physique, Paris, France, 1987.

[49] Oberhuber R, Zandler G, Vogl P. Appl Phys Lett 1998; $73: 818$.

[50] Di Carlo A, Pescetelli S, Paciotti M, Lugli P, Graf M. Solid State Commun 1996;98:803.

[51] Di Carlo A. Phys Stat Solidi, in press; Della Sala F, Di Carlo A, Lugli P, Bernardini F, Fiorentini V, Scholz R, et al. Appl Phys Lett 1999;74:2002.

[52] Cingolani R, Botchkarev A, Tang H, Morkoç H, Coli' G, Lomascolo M, et al. P. Phys Rev B 11(4):2711-15.

[53] Bonfiglio A, Lomascolo M, Traetta G, Cingolani R, Di Carlo A, Della Sala F, et al. J Appl Phys, in press.

[54] Lugli P, Paciotti M, Calleja E, Munoz E, Sanchez-Rojas JJ, Dessenne F, et al. HEMT models and simulations. In: Lee Ross R, Swensson S, Lugli P, editors. Pseudomorphic HEMTs: technology and applications. Dordrecht: Kluwer; 1996. p. 141-63.
[55] Fiorentini V, Bernardini F, Della Sala F, Di Carlo A, Lugli A. Phys Rev B 1999;60:8849.

[56] Trellakis A, Halick AT, Pacelli A, Ravaioli U. J Appl Phys 1997;81:7880.

[57] Lee K, Shur MS, Drummond TJ, Morkoç H. Parasitic MESFET in (Al,Ga)As/GaAs modulation doped FETs and MODFET characterization. IEEE Trans Electron Dev 1984;ED-31:29-35.

[58] Yu ET, Sullivan GJ, Asbeck PM, Wang CD, Qiao D, Lau SS. Measurement of the piezoelectrically induced charge in GaN/AlGaN heterostructure field-effect transistors. Appl Phys Lett 1997;71:2794.

[59] Ramvall P, Aoyagi Y, Kuramata A, Hacke P, Horino K. Influence of a piezoelectric field on the electron distribution in a double $\mathrm{GaN} / \mathrm{Al}_{0.14} \mathrm{Ga}_{0.86} \mathrm{~N}$ heterojuction. Appl Phys Lett 1999;74:3866.

[60] Gaska R, Yang JW, Osinsky A, Bykhovski AD, Shur MS. Piezoeffect and gate current in AlGaN/GaN high electron mobility transistors. Appl Phys Lett 1997;71:3673.

[61] Gaska R, Yang JW, Osinsky A, Bykhovski AD, Shur MS, Kaminski VV, et al. The influence of the deformation on the two-dimensional electron gas density in GaN-AlGaN heterostructure. Appl Phys Lett 1998;72:64.

[62] Carnez B, Cappy A, et al. Modeling of a submicrometer gate field effect transistor including effects of nonstationary electron dynamics. J Appl Phys 1980;51:784-90.

[63] Sandborn PA et al. Quasi-two-dimensional modelling of GaAs MESFETs. IEEE Trans Electron Dev 1987;ED34:985-91.

[64] Snowden CM et al. Quasi-two-dimensional modelling MESFET simulation for CAD. IEEE Trans Electron Dev 1989;ED-36:1564-73.

[65] Morkoç H. GaN-Based modulation doped FETs and UV detectors. Naval Res Rev 1999;51(1):28-45.

[66] Murphy MJ, Foutz BE, Chu K, et al. MRS Internet J Nitride Semicond Res 1999;4S1:G84.

[67] Yu YF, Keller BP, Keller S, Kapolnek D, Kozodoy P, Denbaars SP, et al. Appl Phys Lett 1996;69:1438.

[68] Weitzel C, Pond L, Moore K, Bhatnagar M. Effect of device temperature on RF FET power density. Proc. of Silicon Carbide, III-Nitrides and Related Materials, ICSI, August 1997, Stockholm, Sweden, Trans Publications; Mater Sci Forum 1998;264-268:907-12.

[69] Suzue K, Mohammad SN, Fan ZF, Kim W, Aktas Ö, Botchkarev AE, et al. Electrical conduction in platinumgallium nitride Schottky diodes. J Appl Phys 1996; 80(6):4467-78.

[70] Mohammad SN, Fan Z, Botchkarev AE, Kim W, Aktas Ö, Salvador A, et al. Near ideal platinum-GaN Schottky diodes. Electron Lett 1996;32:598.

[71] Aktas Ö, Kim W, Fan Z, Mohammad SN, Botchkarev A, Salvador A, et al. High transconductance-normallyoff GaN MOD-FETs. Electron Lett 1995;31(16):138990.

[72] Aktas Ö, Fan Z, Botchkarev A, Roth M, Jenkins T, Kehias LT, et al. Microwave performance of $\mathrm{AlGaN} / \mathrm{GaN}$ inverted MODFETs. IEEE Electron Dev Lett 1997;18: 293-5.

[73] Hacke P, Detchprohm T, Hiramatsu K, Sawaki N. Schottky barrier on n-type GaN grown by hydride vapor phase epitaxy. Appl Phys Lett 1993;63:2676-8. 
[74] Binari SC, Dietrich HB, Kelner G, Roland LB, Doverspike K, Gaskill DK. Electrical characterisation of Ti Schottky barriers on n-type GaN. Electron Lett 1994;30: 909-10.

[75] Khan MA, Van Hove JM, Kuznia JN, Olson DT. High electron mobility $\mathrm{GaN} / \mathrm{Al}_{x} \mathrm{Ga}_{1-x} \mathrm{~N}$ heterostructures grown by low-pressure metalorganic chemical vapor deposition. Appl Phys Lett 1991;58:2408-10.

[76] Khan MRH, Nakayama H, Detchprohm T, Hiramatsu K, Sawaki N. A study on barrier height of $\mathrm{Au}-\mathrm{Al}_{x} \mathrm{Ga}_{1-x} \mathrm{~N}$ Schottky diodes in the range of $0<x<0.20$. Topical Workshop on III-V Nitrides Proc., Nagoya, Japan, 1995; Solid State Electron 1997;41(2):259-66.

[77] Foresi JS, Moustakas TD. Metal contacts to gallium nitride. Appl Phys Lett 1993;62:2859-61.

[78] Khan MA, Kuznia JN, Bhattarai AR, Olson DT. Metal semiconductor field-effect transistor based on single-crystal GaN. Appl Phys Lett 1993;62:1786-7.

[79] Lin ME, Ma Z, Huang FY, Fan Z, Allen L, Morkoç H. Low resistance ohmic contacts on wide band-gap GaN. Appl Phys Lett 1994;64:1003-5.

[80] Wu Y, Jiang W, Keller B, Keller S, Kapolnek D, Denbaars $\mathrm{S}$, et al. Low resistance ohmic contacts to $\mathrm{n}-\mathrm{GaN}$ with a separate layer method. Proc Solid State Electron 1997;41(2):75-8.

[81] Fan Z, Mohammad SN, Kim W, Aktas Ö, Botchkarev AE, Morkoç H. Very low resistance multi-layer ohmic contact to n-GaN. Appl Phys Lett 1996;68:1672-4.

[82] Ruvimov S, Liliental-Weber Z, Washburn J, Duxstad KJ, Haller EE, Mohammad SN, et al. Microstructure of Ti/Al and $\mathrm{Ti} / \mathrm{Al} / \mathrm{Ni} / \mathrm{Au}$ ohmic contacts for $\mathrm{n}-\mathrm{GaN}$. Appl Phys Lett 1996;69(11):1556-8.

[83] Moloney M, Ponse F, Morkoç H. Gate capacitance voltage characteristics of MODFETs: its effect on transconductance. IEEE Trans Electron Dev 1985;ED-32(9): 1675-84.

[84] Lin ME, Strite S, Agarwal A, Salvador A, Zhou GL, Teraguchi N, et al. GaN grown on hydrogen plasma cleaned 6H-SiC substrates. Appl Phys Lett 1993;62(7): $702-4$.

[85] Powell JA, Larkin DJ, Trunek AJ. Use of gaseous etching for the characterization of structural defects in silicon carbide single crystals. In: Pensl G, Morkoç H, Monemar B, Janzen E, editors. Silicon carbide, III-nitrides, and related materials, vols. 264-268. Sweden: Trans Tech Publications; 1998. p. 421-424.

[86] Powell JA, Larkin DJ, Neudeck PG, Yang JW, Pirouz P. Investigation of defects in epitaxial $3 \mathrm{C}-\mathrm{SiC}, 4 \mathrm{H}-\mathrm{SiC}$ and $6 \mathrm{H}-\mathrm{SiC}$ films grown on $\mathrm{SiC}$ substrates. In: Spencer MG, Devaty RP, Edmond JA, et al., editors. Silicon carbide and related materials. Bristol: IOP Publishing; 1994. p. 161-4.

[87] Moore KE, Weitzel CE, Nordquist KJ, Pond III LL, Palmour JW, Allen S, et al. IEEE Electron Dev Lett 1997;18(2):69-70.

[88] Vincent D. Fundamentals of infrared detector operation and testing. New York: Wiley; 1990.

[89] Kruse PW, McGlauchlin LD, McQuistan RB. Elements of infrared technology. New York: Wiley; 1962.

[90] Rose A. Concepts in photoconductivity and allied problems. New York: Interscience; 1963.

[91] Nelson RD. Opt Eng 1977;16:275.
[92] Prabhakaran K, Anderson TG, Nozawa K. Appl Phys Lett 1996;69:3212.

[93] Chen Q, Asif Khan M, Sun CJ, Yang JW. Visible-blind ultraviolet photodetectors based on GaN p-n junctions. Electron Lett 1995;31:1781-2.

[94] Walker D, Zhang X, Kung P, Saxler A, Javadpour S, Xu J, et al. AlGaN ultraviolet photoconductors grown on sapphire. Appl Phys Lett 1996;68:2100-1.

[95] Stevens KS, Kinniburgh M, Beresford R. Photoconductive ultraviolet sensor using $\mathrm{Mg}$-doped $\mathrm{GaN}$ on $\mathrm{Si}(111)$. Appl Phys Lett 1995;66:3518-20.

[96] Wickenden D, Huang Z, Mott DB, Shu PK. Development of gallium nitride photoconductive detectors. Johns Hopkins APL Tech Digest 1997;1S:217-25.

[97] Chen Q, Yang JW, Osinsky A, Gangopadhyay S, Lim B, Anwar MZ, et al. Schottky barrier detectors on $\mathrm{GaN}$ for visible-blind ultraviolet detection. Appl Phys Lett 1997;70:2277-9.

[98] Van Hove JM, Hickman R, Klaassen JJ, Chow PP. Ultraviolet-sensitive, visible-blind GaN photodiodes fabricated by molecular beam epitaxy. Appl Phys Lett 1997;70:2282-4.

[99] Osinsky A, Gangopahyay S, Gaska R, Williams B, Khan MA, Kuksenkov D, et al. Low noise p-p-n GaN ultraviolet photodetectors. Appl Phys Lett 1997;71:2334-6.

[100] Walker D, Zhang X, Kung P, Saxler A, Javadpour S, Xu J, et al. $\mathrm{Al}_{x} \mathrm{Ga}_{1-x} \mathrm{~N}$ ultraviolet photoconductors grown on sapphire by metal-organic chemical vapor deposition. Appl Phys Lett 1997;70:949-51.

[101] Lim BW, Chen QC, Yang JY, Asif Khan M. High responsivity intrinsic photoconductors based on $\mathrm{Al}_{x} \mathrm{Ga}_{1-x} \mathrm{~N}$. Appl Phys Lett 1996;68:3761.

[102] Binet F, Duboz JY, Rosencher E, Scholz F, Härle V. Mechanism of recombination in $\mathrm{GaN}$ photodetectors. Appl Phys Lett 1996;69:1202-4.

[103] Misra M, Korakakis D, Singh R, Sampath A, Moustakas TD. Photoconducting properties of ultraviolet detectors based on GAN and $\mathrm{Al}_{x} \mathrm{Ga}_{1-x} \mathrm{~N}$ grown by ECR-assisted MBE. In: Ponce FA, Moustakas TD, Akasaki I, Monemar BA, editors. MRS Proc, vol. 449. 1997. p. 597.

[104] Binet F, Duboz JY, Rosencher E, Briot O, Aulombard RL. Properties of a photovoltaic detector based on an n-type GaN Schottky barrier. J Appl Phys Lett 1997;81: 449-54.

[105] Munoz E, Monroy E, Carrido JA, Izpura I, Sanchez FJ, Garcia MA, et al. Photoconductor gain mechanism in GaN ultraviolet detectors. Appl Phys Lett 1997;71:870-2.

[106] Smith G, Estes MJ, Tang D, Salvador A, Fan Z, Xu G, et al. Megahertz bandwidth $\mathrm{Al}_{x} \mathrm{Ga}_{1-x} \mathrm{~N} / \mathrm{GaN}$-based pin detector, presented at the SPIE meeting, San Jose CA, 25-28 January, 1998.

[107] Campbell JC. University of Texas at Austin, private communication.

[108] Carrano JC, Li T, Brown DL, Grudowski PA, Eiting CJ, Dupuis RD, et al. High-speed pin ultraviolet photodetectors fabricated on GaN. Electron Lett 1998;34(18):177981.

[109] Carrano JC, Grudowski PA, Elting CJ, Dupuis RD, Campbell JC. Very low dark current metal-semiconductor-metal ultraviolet photodetectors fabricated on single-crystal GaN epitaxial layers. Appl Phys Lett 1997;70:1992-4. 
[110] Carrano JC, Li T, Grudowski PA, Eiting CJ, Dupuis RD, Campbell JC. Comprehensive characterization of metalsemiconductor-metal ultraviolet photodetectors fabricated on single-crystal GaN. J Appl Phys 1998;83(11 and 1):6148-60.

[111] Walker D, Monroy E, Kung P, Wu J, Hamilton M, Sanchez FJ, et al. High-speed, low-noise metal-semiconductor-metal ultraviolet photodetectors based on GaN. Appl Phys Lett 1999;74(5 and 1):762-4.

[112] Campbell JC. University of Texas at Austin, private communication.
[113] Lim W, Gangopadhyay S, Yang JW, Osinsky A, Chen Q, Anwar MZ, et al. $8 \times 8 \mathrm{GaN}$ Schottky barrier photodiode array for visible-blind imaging. Electron Lett 1997;33(7): 633-4.

[114] Huang ZC, Chen JC, Mott DB, Shu PK. High performance GaN linear array. Electron Lett 1996;32(14): $1324-5$.

[115] Brown JD, Yu Z, Matthews J, Harney S, Boney J, Schetzina JF, et al. Visible-blind UV digital camera based on a $32 \times 32$ array of GaN/AlGaN p-i-n photodiodes. MRS Internet J, http://nsr.mij.mrs.org/4/9/. 